\author{
UnIVERsità DI PISA \\ Dipartimento Di Matematica L.TONELli \\ Scuola di DotTorato
}

Ph.D. Thesis

\title{
Outer Approximation Algorithms for DC programs and beyond
}

\author{
QINGHUA ZHANG
}

Advisors: Proff. Giancarlo Bigi And Antonio Frangioni

School Director: Prof. Fabrizio Broglia 



\section{Acknowledgements}

This thesis is the result of four years of work whereby I have been accompanied and supported by many people. It is my pleasure that I have now the opportunity to express my gratitude to all of them. Without them, this dissertation would not have been possible.

I would like to express my deep and sincere gratitude to my supervisors, Professors Giancarlo Bigi and Antonio Frangioni. Their wide knowledge and logical way of thinking have been of great value for me. Their understanding, encouraging and personal guidance have provided a good basis for the present thesis. I thank them for their patience and encouragement that carried me on through difficult times, and for their insights and suggestions that helped to shape my research skills. Their valuable feedback contributed greatly to this dissertation.

I am deeply grateful to my former supervisor, Professor Stefano Pallottino. His perpetual energy and enthusiasm in research had motivated me for all the time. Although he is no longer with us, he is forever remembered. I owe my most sincere gratitude to Professor Giorgio Gallo, he and Professor Stefano Pallottino introduced me to this operations research group four years ago. Joining this group was not only a turning point in my life, but also a wonderful experience. I cherished the friendship and support. I treasured all precious moments we shared and would really like to thank them. I am also wish to thank Professor Maria Grazia Scutellà, Fulvio Piccinonno, and colleagues Lorenzo Cioni, Fausto Pascali, Luis Perez, Raffaella Recchia, and Claudia Sodini.

I would like to thank Prof Fabrizio Broglia, the Galilei Office and all the staffs in Departments of Mathematics and Computer Science, they helped me a lot so many times. I should also thank the financial support of the University of Pisa.

I feel a deep sense of gratitude for my father and mother who formed part of my vision and taught me the good things that really matter in life. I am grateful to my brother Zhang Songhua and his family for rendering me the value

of brotherhood. I am very grateful to my wife Yang Shan for her love and patience during the $\mathrm{PhD}$ period. 



\section{Contents}

1 DC Optimization 1

1.1 DC Functions . . . . . . . . . . . . . . . . . . . . 1

1.2 DC Sets . . . . . . . . . . . . . . . . . 5

1.3 DC Optimization Problems . . . . . . . . . . . . . 8

1.3.1 The Canonical Form of DC Problems . . . . . . . . . . 10

1.4 Literature Review . . . . . . . . . . . . . . . . . . . . . . . . . . . 18

1.4.1 Concave Minimization Problems . . . . . . . . . . . . 18

1.4.2 Concave Minimization Problems with a Reverse Convex Constraint . . . . . . . . . . . . . . . 21

1.4.3 Reverse Convex Optimization Problems . . . . . . . . . . . 22

1.4.4 General Constrained DC Optimization Problems . . . . . . 24

1.4.5 Canonical DC Optimization Problems . . . . . . . . . 25

2 Algorithms for Canonical DC Problems 29

2.1 Introduction . . . . . . . . . . . . . . . . . . . 29

2.2 Approximate Optimality Conditions . . . . . . . . . . . . . . . 30

2.2.1 Optimality Conditions and (Approximate) Oracles . . . . . 30

2.2.2 Approximate Optimality Conditions . . . . . . . . . . 32

2.3 Conditions and Algorithms . . . . . . . . . . . . . . . . 37

2.3.1 General Convergence Conditions . . . . . . . . . . . . . . . 37

2.3.2 The Outer Approximation Machinery . . . . . . . . . . . . 41

2.3.3 A Generic Outer Approximation Sub-Procedure . . . . . . 43

2.3.4 Algorithms Exploiting the Set of Conditions $B_{1}$. . . . . . 46

2.3.5 Algorithms Exploiting the Set of Conditions $B_{2}$. . . . . . 54

2.3.6 Summary ................. 56 
2.4 Comparisons and Conclusions . . . . . . . . . . . . . . 60

3 Algorithms for Single Reverse Polar Problems 63

3.1 Introduction . . . . . . . . . . . . . . . . . 63

3.2 The Single Reverse Polar Problem . . . . . . . . . . . . . . . . . . 65

3.3 Approximate Optimality Conditions . . . . . . . . . . . . . 66

3.3.1 Optimality Conditions and (Approximate) Oracles . . . . . 66

3.3.2 Properties of Optimal Solutions . . . . . . . . . . . 68

3.3.3 Approximate Optimality Conditions . . . . . . . . . . 70

3.4 Conditions and Algorithms . . . . . . . . . . . . . . . 76

3.4.1 General Convergence Conditions . . . . . . . . . . . 76

3.4.2 The Outer Approximation Machinery . . . . . . . . . . . 78

3.4.3 A Generic Outer Approximation Sub-Procedure . . . . . . 79

3.4.4 Implementable algorithms . . . . . . . . . . . . . . . . 84

3.5 Conclusions . . . . . . . . . . . . . . . . . . . . . 100

4 Conclusions and directions for future work 103 


\section{Introduction}

Nonconvex optimization problems often arise from applications in engineering, economics and other fields (see for instance [20]). A large number of them are actually DC optimization problems, that is nonconvex problems where the objective function is the difference of two convex functions and the constraint can be expressed as the set difference of two convex sets. In particular, the Canonical DC (shortly $C D C$ ) problem has been investigated in many papers, as every DC optimization problem can be reduced to a Canonical DC problem through standard transformations (see [101]). Several algorithms to solve it have been proposed (see, for instance, [102, 91, 72, 95, 90, 28]) and generally they are modifications of the first cutting plane algorithm proposed by Tuy in [102].

In this thesis, we consider the Canonical DC problem relying on an alternative equivalent formulation based on a polar characterization of the constraint. The structure of this formulation allows to carry out a thorough analysis of convergence for cutting plane type algorithms. Different sets of conditions, which guarantee convergence, are proposed and exploited to build six algorithms, one of which is improved Tuy's algorithm [102], another one follows the same general procedure but takes an improved stopping criteria, and the other four are new and can't be reduced to the original cutting plane algorithm in [102] by Tuy.. Furthermore, the alternative formulation allows to define proper approximate optimality conditions, which can be exploited to guarantee that the algorithms end after a finite number of iterations and provide approximate global optimal solutions.

Afterwards, we propose a novel generalization of the Canonical DC problem and we study the convergence of outer approximation (cutting planes) algorithms for its solution, which use an "approximated" oracle for checking the global 
optimality conditions to the problem. Although the approximated optimality conditions are similar to those of the Canonical DC problem, the new class of Single Reverse Polar problems is shown to significantly differ from its special case. We also show that outer approximation approaches for DC problems need be substantially modified in order to cope with Single Reverse Polar problem. We develop a hierarchy of conditions that guarantee the convergence of cutting plane algorithms; relying on these conditions, we build three cutting plane algorithms for solving Single Reverse Polar problem, which seem to be new and cannot be reduced to each other.

The thesis is organized as follows. In Chapter 1, DC functions, DC sets and the Canonical DC problem are introduced, and the well-known optimality conditions are recalled. Then we give a literature review on solution methods for solving DC problems and the other related global optimization problems. Chapter 2 proposes approximate optimality conditions and investigates the relationship between the exact optimal value and the approximate optimal values. Then convergence analysis is carried out and six different algorithms are proposed and the corresponding proofs of finite termination are given. At the end, the connections of these results with the existing algorithms are outlined. In Chapter 3 we analyze the main properties of the Single Reverse Polar problem and contrast them with those of its special case Canonical DC. Then we extend our approximate optimality conditions for Canonical DC to the Single Reverse Polar case. We also develop a hierarchy of conditions that guarantee the convergence of cutting plane algorithms; relying on these conditions, we build three cutting plane algorithms for solving the Single Reverse Polar problem, which seem to be new and cannot be reduced to each other. 


\section{Chapter 1}

\section{Optimization}

In this chapter, we shall give an overview of DC optimization problems, which includes the definitions and properties of DC functions, DC sets, DC optimization problems with the corresponding optimality conditions. Then we give a literature review on the solution methods for solving DC optimization problems and related problems, such as concave minimization problems and reverse convex problems.

\subsection{Functions}

DC functions widely exist in optimization problems: almost all type of functions can be represented or approximated by DC functions; furthermore, the family of DC functions is stable under many operations. All the above suggest that DC optimization problems may play an important role in global optimization problems.

Definition 1.1.1 A real-valued function $f$ defined on a convex set $\Omega \subseteq \mathbb{R}^{n}$ is called DC on $\Omega$ if $f$ can be expressed as the difference of two convex functions for all $x \in \Omega$, namely

$$
f(x)=p(x)-q(x)
$$

where $p$ and $q$ are convex functions on $\Omega$. A function that is DC on $\mathbb{R}^{n}$ is called DC. The representation (1.1) is said to be a DC representation of $f$.

Definition 1.1.2 A real-valued function $f: \Omega \rightarrow \mathbb{R}$ defined on a convex set 
$\Omega \subseteq \mathbb{R}^{n}$ is said to be locally DC if for every $x_{0} \in \Omega$ there exists a neighborhood

$$
U\left(x_{0}, \varepsilon\right)=\left\{x \in \Omega \mid\left\|x-x_{0}\right\|<\varepsilon\right\}, \varepsilon>0
$$

such that $f$ is $\mathrm{DC}$ on $U\left(x_{0}, \varepsilon\right)$.

DC functions are sometimes denoted as $\delta$-convex or $\Delta$-convex functions [21]. However, $\delta$-convex functions are also used for "almost convex" functions with an error at most $\delta>0[21]$. This is the reason why we prefer the name "DC function" as the formal one.

DC functions of one variable were considered by mathematicians to characterize the indefinite integrals of functions with locally bounded variation [21]. Alexandroff [1] was the first to consider the DC functions with many variables. In 1959, Hartman [32] defined the DC mappings between Euclidean spaces as mappings with DC components and proved that the composition of two DC mappings is DC. Hiriart Urruty [35] gives an excellent survey of the main known results on DC functions and related optimization problems.

The class of DC functions is a linear space generated by convex functions. As it is well known, convexity and concavity are not preserved under some simple algebraic operations, i.e. for any strictly convex (or concave) function $f,(-1 \cdot f)$ is not convex (or concave). Unlike convex and concave functions, DC functions are closed with respect to many operations frequently used in optimization such as scalar multiplication, lower and upper envelope, function composition, product and quotient.

Proposition 1.1.1 [113, Proposition 4] Let $\Omega_{1} \subseteq \mathbb{R}^{n}$ and $\Omega_{2} \subseteq \mathbb{R}^{m}$ be convex sets such that $\Omega_{1}$ is open or closed and $\Omega_{2}$ is open. If $F_{1}: \Omega_{1} \rightarrow \Omega_{2}, F_{2}: \Omega_{2} \rightarrow \mathbb{R}^{k}$ are DC mappings, then $F_{2} \circ F_{1}: \Omega_{1} \rightarrow \mathbb{R}^{k}$ is also a DC mapping.

The set $\Omega_{2}$ must be open or closed in Proposition 1.1.1, otherwise $F_{2} \circ F_{1}$ may not be $\mathrm{DC}$ on $\Omega_{1}$. A counter-example can be found in Hartman [32].

Proposition 1.1.2 If $f_{i}(x)=p_{i}(x)-q_{i}(x)$ are DC functions on $\Omega$ where $p_{i}$ and $q_{i}$ are convex functions for $i=1,2, \ldots, m$. Then the following functions are also $D C$ on $\Omega$ :

1) $\sum_{i=1}^{m} \alpha_{i} f_{i}(x)$, for any real numbers $\alpha_{i}$, 
2) $g(x)=\max \left\{f_{1}(x), \ldots, f_{m}(x)\right\}$ and $h(x)=\min \left\{f_{1}(x), \ldots, f_{m}(x)\right\}$,

3) $\left|f_{i}(x)\right|, f^{+}(x):=\max \{0, f(x)\}, f^{-}(x):=\min \{0, f(x)\}$,

4) the product $\prod_{i=1}^{m} f_{i}(x)$ and the quotient $f_{1}(x) / f_{2}(x)$ if $f_{2}(x) \neq 0$ on $\Omega$.

Proof.1) is trivial, let's prove 2): By the definition of $f_{i}$ we have

$$
f_{i}=p_{i}(x)+\sum_{j \neq i} q_{j}(x)-\sum_{j=1}^{m} q_{j}(x),
$$

which leads to the following equations:

$$
\begin{aligned}
& g(x)=\max _{i=1,2, \ldots, m}\left\{\left[p_{i}(x)+\sum_{j \neq i} q_{j}(x)\right]\right\}-\sum_{j=1}^{m} q_{j}(x), \\
& h(x)=\sum_{j=1}^{m} p_{j}(x)-\max _{i=1,2, \ldots, m}\left\{\left[\sum_{j \neq i} p_{j}(x)+q_{i}(x)\right]\right\} .
\end{aligned}
$$

Therefore, $g$ and $h$ are DC on $\Omega$ since the sum and maximum of a family of convex functions are still convex.

3): The function 0 is DC, then $f^{+}$and $f^{-}$are $\mathrm{DC}$ on $\Omega$ and thus $|f|$ is DC on $\Omega$ since $|f|=f^{+}-f^{-}$.

4): It suffices to show that $f_{1} \cdot f_{2}$ is DC for any two DC functions $f_{1}$ and $f_{2}$ : Let $F_{1}=\left(f_{1}, f_{2}\right): \Omega \rightarrow \mathbb{R}^{2}$ and $F_{2}\left(x_{1}, x_{2}\right)=x_{1} x_{2}: \mathbb{R}^{2} \rightarrow \mathbb{R}$, then $F_{1}$ is DC on $\Omega$ and $F_{2}=\frac{1}{2}\left[\left(x_{1}+x_{2}\right)^{2}-\left(x_{1}^{2}+x_{2}^{2}\right)\right]$ is DC on $\mathbb{R}^{2}$. Proposition 1.1.1 guarantees the DC property of $F_{2} \circ F_{1}=f_{1} \cdot f_{2}$ on $\Omega$.

Furthermore, $\frac{1}{f(x)}$ is DC on $\Omega$ for any function $f(x) \in D C(\Omega)$ such that $f(x) \neq$ 0 on $\Omega$ : as the function $g(y)=\frac{1}{y}$ is $\mathrm{DC}$ on $\mathbb{R}$ when $y \neq 0\left(C^{2}\right.$-smooth functions are DC), Proposition 1.1.1 guarantees that the function $g(f(x))=1 / f(x)$ is also $\mathrm{DC}$ on $\Omega$.

Many frequently encountered functions are DC or can be approximated by DC functions: all of the convex and concave functions are DC; In particular, the class of DC functions contains all twice differentiable $C^{2}$ functions (see Lemma 1.1.1). However, although DC functions occur frequently in practice, they often appear in a hidden way and their DC structures are often not given explicitly. In order to transform any optimization problem into DC form, it is important to 
identify the DC property of the functions. The following proposition helps us to identify DC functions by exploring their local properties.

Proposition 1.1.3 [113, Proposition 3] A locally DC function $f$ on a convex, closed or open, set $D$ is $D C$ on $D$.

Then we can judge the DC property by using some local properties with Proposition 1.1.3. One possible way is to detect the differentials.

Definition 1.1.3 A real-valued function $f: \Omega \rightarrow \mathbb{R}$ is a $C^{2}$ mapping if $\nabla^{2} f$ exists at each point of $\Omega$ and $\nabla^{2} f$ is continuous; furthermore, $f$ is a $C^{1,1}$ mapping if its gradient $\nabla f$ exists at each point $x$ of $\Omega$ and $\nabla f$ satisfies the locally Lipschitz condition on $\Omega$, namely, there exists $L>0$ such that

$$
\|\nabla f(x)-\nabla f(y)\| \leq L\|x-y\| \quad \forall x, y \in \Omega
$$

Lemma 1.1.1 [113, Proposition 2] Every function $f \in C^{2}(\Omega)$ is $D C$ on any compact convex set $\Omega$.

Proof. It suffices to show that there exists sufficiently large $\lambda>0$ such that the function $g(x)=f(x)+\frac{1}{2} \lambda\|x\|^{2}$ is convex on $\Omega$ : choose any $\lambda$ s.t.

$$
-\min \left\{u \nabla^{2} f(x) u \mid x \in \Omega,\|u\|=1\right\} \leq \lambda
$$

then $u \nabla^{2} g(x) u=u \nabla^{2} g(x) u+\lambda\|u\|^{2} \geq 0$ for any $u$. Therefore, the function $g$ is convex.

Moreover, any $C^{1,1}$ function is also DC (see [35]); anyway the proof involves advanced tools from functional analysis. Since a polynomial has continuous derivatives of any order, we have the following consequence of the above lemma.

Corollary 1.1.1 Any polynomial in $x \in \mathbb{R}^{n}$ is a DC function on $\mathbb{R}^{n}$.

Lemma 1.1.1 shows that a function is DC on any compact convex, set $\Omega$, thus any continuous function on such a set can be approximated by a DC function since it can be approximated by a polynomial function in $\mathbb{C}^{2}(\Omega)[113]$. Let $C(\Omega)$ be the Banach Space of continuous functions on $\Omega$, we get that $D C(\Omega)$ is dense in $C(\Omega)$ equipped with the sup norm. 


\subsection{Sets}

This section provides the definitions of DC set and DC inequality, then shows that all sets defined by a DC inequality in $\mathbb{R}^{n}$ can be seen as the projection of a DC set in $\mathbb{R}^{n+1}$. In particular, this section provides several ways to define closed sets by DC inequalities.

Definition 1.2.1 A set $M \subseteq \mathbb{R}^{n}$ is called a DC set if it can be described in the form $M=D \backslash C$ where $D$ and $C$ are two convex sets in $\mathbb{R}^{n}$.

A DC set can be described as a difference of two convex sets, while a DC function can be described as a difference of two convex functions. Then a question arises: given any real-valued DC function $f$, is there any relationship between

the set $\left\{x \in \mathbb{R}^{n} \mid f(x) \leq 0\right\}$ and DC sets? The answer is positive. Then it is natural to introduce DC inequalities.

Definition 1.2.2 An inequality of the form $f(x) \leq 0$ is convex when $f$ is convex. If $f$ is concave, then this inequality is called reverse convex. If $f$ is $\mathrm{DC}$, then this inequality is called DC inequality.

It should be noted that, when $f$ is DC, $f(x) \geq 0$ is still a DC inequality since $-f$ is also DC. Proposition 1.1.2 guarantees that DC functions are closed under lower and upper envelopes. Thus any finite system of DC inequalities, whether conjunctive or disjunctive, is equivalent to a single DC inequality. Furthermore, by introducing an additional variable $t$, the DC inequality $p(x)-q(x) \leq 0$ where $p$ and $q$ are convex can be split into two inequalities:

$$
p(x)-t \leq 0, \quad t-q(x) \leq 0
$$

where the first is a convex inequality and the second is a reverse convex inequality. By this property, we will see that any DC optimization problem can be reduced to a canonical form (see Section 1.3.1).

Lemma 1.2.1 Any closed set can be defined by a DC inequality.

Proof. For any closed set $S \subseteq \mathbb{R}^{n}$, let

$$
d(x, S)=\inf \{\|x-y\| \mid y \in S\}
$$


denote the distance from $x$ to $S$, then the function

$$
x \mapsto\|x\|^{2}-d^{2}(x, S)=\sup \left\{2 x y-\|y\|^{2} \mid y \in S\right\}
$$

is convex since it is the supremum of a family of affine functions. Thus $S$ is defined by the inequality $d^{2}(x, S) \leq 0$ and $d^{2}(x, S)$ is a DC function.

Lemma 1.2.1 provides an applicable but not very convenient way to construct a DC inequality for any closed set. As the corresponding DC inequality of any DC set is not unique, we will introduce some other better ways in this section.

Remark 1.2.1 While it is not easy to find a DC inequality of a general closed set, the DC inequality of a DC set can be easily obtained in the following particular case: where convex sets $D$ and $C$ are closed and open, respectively. And $D=\{x \mid g(x) \leq 0\}$ and $C=\{x \mid h(x)<0\}$ where $g$ and $h$ are closed convex functions, then $M=D \backslash C$ can be expressed as $M=\{x \mid g(x) \leq 0, h(x) \geq 0\}$. Therefore, the DC set $M$ can also be defined by a DC inequality:

$$
M=\{x \mid \max \{g(x),-h(x)\} \leq 0\} .
$$

A DC set can be defined by a DC inequality, but a set defined by DC inequality may not be DC, as the following example shows.

Example 1.2.1 Let $S=\left\{x \mid x^{2} \leq 4\right\} \cup\left\{x \mid(x-4)^{2} \leq 4\right\} \cup\left\{x \mid(x+4)^{2} \leq 4\right\}$ as depicted in Figure 1.1. $S$ is closed and therefore it can be defined by a DC inequality. Let $S=D \backslash C$, it is obvious that the points $(0,2),(-4,2)$ and $(4,2)$ belong to $D$ since they belong to $S$. This implies that the points $(-2,2)$ and $(2,2)$ belong to $D$ since $D$ is convex. But $(-2,2)$ and $(2,2)$ don't belong to $S$, then we get that $(-2,2)$ and $(2,2)$ also belong to $C$. Therefore, the point $(0,2)$ also belongs to $C$ since $C$ is convex, a contradiction.

Example 1.2.1 shows that a set defined by a DC inequality may not be DC. Then the question is: does any set defined by a DC inequality has a close relationship with the DC sets? The answer is positive.

Lemma 1.2.2 Any set defined by a DC inequality can be seen as the projection on $\mathbb{R}^{n}$ of a DC set in $\mathbb{R}^{n+1}$. 


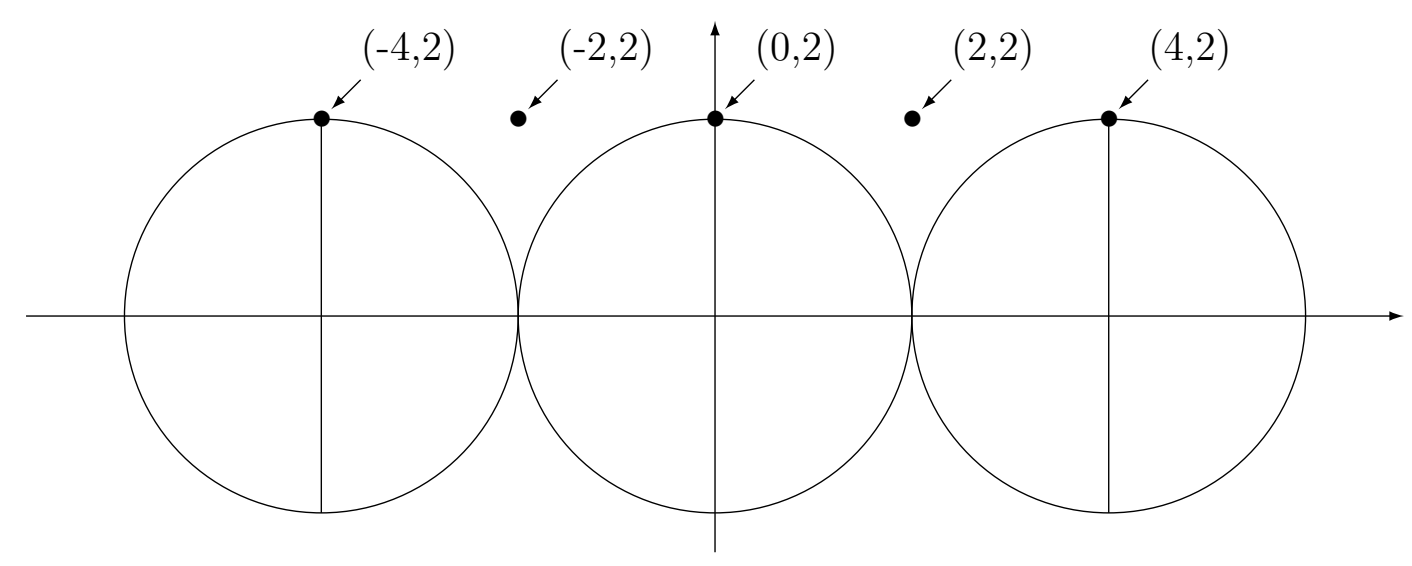

Figure 1.1: A non-DC set that can be defined by a DC inequality.

Proof. Given a set $\Omega=\{x \mid g(x)-h(x) \leq 0\}$ where $g$ and $h$ are convex, let

$$
M=\{(x, t) \mid g(x)-t \leq 0, t-h(x) \leq 0\}
$$

It is obvious that $M$ is a DC set in $\mathbb{R}^{n+1}$ and that $\Omega$ is the projection of $M$.

Lemma 1.2.2 implies that any closed set can be seen as the projection of a DC set in $\mathbb{R}^{n+1}$. Then the problem is how to express closed sets by DC inequalities. Although Lemma 1.2.1 provides a way to express any closed set by a DC inequality, it is difficult to compute the function (1.3). Furthermore, $d(x, S)=0$ for all $x \in S$, so the representation $S=\left\{x \mid d^{2}(x, S)=0\right\}$ doesn't make difference between interior and boundary of $S$, in fact, int $S \neq\left\{x \mid d^{2}(x, S)<0\right\}$. Moreover, $d(x, S)$ is difficult to construct. Therefore, we aim to find the type of convenient DC functions that define any closed set.

Definition 1.2.3 Let $h: \mathbb{R}^{n} \rightarrow \mathbb{R}$ be a strictly convex function, $S$ a nonempty closed set in $\mathbb{R}^{n}$. Define

$$
d^{2}(x)=\inf _{y \in S, p \in \partial h(x)}[h(y)-h(x)-p(y-x)]
$$

where $\partial h\left(x^{0}\right)$ denotes the sub-differential of a function at point $x^{0}$, namely the set

$$
\partial h\left(x^{0}\right)=\left\{p \in \mathbb{R}^{n} \mid h(x)-h\left(x^{0}\right) \geq p\left(x-x^{0}\right), \forall x \in \mathbb{R}^{n}\right\} .
$$


This function has the following property.

Lemma 1.2.3 [113, Lemma 3] $(i) d(x)=0, \forall x \in S$,

(ii) $d(x)>0, \quad \forall x \notin S$,

(iii) if $x^{k} \rightarrow x, d\left(x^{k}\right) \rightarrow 0(k \rightarrow \infty)$, then $x \in S$.

The equation (1.4) is better than (1.3) in the sense that (1.4) can make difference between the boundary and interior of any closed set, and it leads to the following proposition.

Definition 1.2.4 Let $\theta$ be any positive number and $r: \mathbb{R}^{n} \rightarrow \mathbb{R}_{+}$any function such that

$$
\begin{gathered}
r(x)=0, \quad \forall x \in S, \\
0<r(y) \leq \min \{\theta, d(y)\}, \quad \forall y \notin S, \\
g_{S}(x)=\sup _{y \notin S, p \in \partial h(y)}\left\{h(y)+p(x-y)+r^{2}(y)\right\} .
\end{gathered}
$$

With the function $r(x), g_{S}$ is proved to be an good definition for any closed set.

Proposition 1.2.1 [113, Proposition 5] Let $h: \mathbb{R}^{n} \rightarrow \mathbb{R}$ be a strictly convex function. For every closed set $S \subset \mathbb{R}^{n}$, the function $g_{S}(x)$ is closed convex and finite everywhere, and satisfies

$$
S=\left\{x \in \mathbb{R}^{n} \mid g_{S}(x)-h(x) \leq 0\right\} .
$$

A function satisfying (1.5) and (1.6) is called a separator. Proposition 1.2.1 shows that any closed set $S$ can be defined by the DC inequality (1.8). In particular, given any closed set $S \subseteq \mathbb{R}^{n}$ and a separator $r$ for $S$, we can describe $S$ as the solution set of the DC inequality $g_{S}(x)-\|x\|^{2} \leq 0$ by taking $h(x)$ as $\|x\|^{2}$. By this inequality, a DC set $M \subseteq \mathbb{R}^{n+1}$ can be obtained such that $S$ is the projection on $\mathbb{R}^{n}$ of $M$.

\subsection{Optimization Problems}

The linear space of DC functions on a convex set $D$ is dense in the space of continuous functions on $D$, the DC structure widely exists in many global optimization problems. In general, a DC optimization problem consists of a $\mathrm{DC}$ objective function and a finite number of DC constraints: 
Definition 1.3.1 A global optimization problem is called a DC programming problem or a DC program if it has the form

$$
\begin{gathered}
\min f_{0}(x) \\
\text { s.t. } \quad f_{i}(x) \leq 0 \quad i=1, \ldots, m, \\
x \in D,
\end{gathered}
$$

where $D$ is a closed convex subset of $R^{n}$ and all functions $f_{i}$ are DC.

How to recognize an optimal solution is important in studying optimization problems. In global optimization problems, it is very difficult to recognize an optimal solution since optimality criterion must be based on the information of the global behavior. There are many forms of DC optimization problems, this section will provide an optimality condition for the following form:

$$
\min g(x)-h(x) \text { s.t } x \in \mathbb{R}^{n} \text {, }
$$

where $g$ is an arbitrary function and $h$ is a lower semi-continuous function. This problem is very general since $g$ and $h$ needn't be convex. In order to study the global optimality condition of problem (1.10), we introduce the concept $\varepsilon$ subdifferential and polar set. The $\varepsilon$-subdifferential of a function $f(x)$ at a point $a$ is the set:

$$
\partial_{\varepsilon} f(a)=\left\{p \in \mathbb{R}^{n} \mid f(x)-f(a) \geq p(x-a)-\varepsilon, \forall x \in \mathbb{R}^{n}\right\},
$$

and the polar of a set $C$ :

$$
C^{*}=\left\{w \in \mathbb{R}^{n} \mid w x \leq 1, \forall x \in C\right\} .
$$

$\partial_{\varepsilon} f$ is related to the local property of function $f$, but it is used to obtain a global optimality condition.

By using the properties of polar sets, we get the following proposition.

Proposition 1.3.1 [113, Proposition 11], [36, Theorem 4.4]

Let $g, h$ be two functions such that $h$ is convex proper lower semi-continuous. Let $\tilde{x}$ be a point where $g$ and $h$ are finite. In order that $\tilde{x}$ be a global minimizer of $g-h$ over $\mathbb{R}^{n}$ it is necessary and sufficient that

$$
\partial_{\varepsilon} h(\tilde{x}) \subseteq \partial_{\varepsilon} g(\tilde{x}), \forall \varepsilon>0
$$


Proof. Denote $\tilde{g}(x)=g(x)-g(\tilde{x})$ and $\tilde{h}(x)=h(x)-h(\tilde{x})$. Then $\tilde{x}$ is the global minimizer if and only if $\tilde{g}(x) \geq \tilde{h}(x)$ for all $x \in \mathbb{R}^{n}$. We can further assume that $\tilde{x}=0$. Let $G, H$ be the epigraph of $\tilde{g}$ and $\tilde{h}$, We get that $G \subseteq H$ is equivalent to $H^{*} \subseteq G^{*}$ since $H$ is closed convex and $0 \in H$.

Take any sub-gradient $p$ from $\partial_{\varepsilon} h(0)$, we have $p \in \partial_{\varepsilon} \tilde{h}(0)$, which is equivalent to $p x-\varepsilon \leq \tilde{h}(x)-\tilde{h}(0)$ or $p x-\tilde{h}(x) \leq \varepsilon$. Pick any $(x, t)$ from $H$, i.e. $t \geq \tilde{h}(x)$, we get that $p \in \partial_{\varepsilon} \tilde{h}(0)$ is equivalent to $p x-t \leq \varepsilon$ and $(p,-1) / \varepsilon \in H^{*}$.

Therefore, $\partial_{\varepsilon} h(\tilde{x}) \subseteq \partial_{\varepsilon} g(\tilde{x})$ for all $\varepsilon>0$ if and only if $H^{*} \subseteq G^{*}$, which is equivalent to the condition $G \subseteq H$, i.e, $\tilde{x}$ is the minimum point of $g-h$.

Proposition 1.3.1 provides a global optimization condition (1.13) for problem (1.10). Although sub-differential is of local property, (1.13) is not a local optimality criterion, because it involves all $\varepsilon$-subdifferentials. The drawback of this condition is that it is hard to check.

Note that when checking whether $\tilde{x}$ is the global minimizer of $g(x)-h(x)$ or not, it is necessary and sufficient that all $x$ satisfying $f(x)=f(\tilde{x})$ are local minimizers [113]. However, as it is shown in Example 1.3.1, $\partial h(x) \subseteq \partial g(x)$ for all $x$ satisfying $f(x)=f(\tilde{x})$ is only a necessary optimality condition, because $\partial h(\tilde{x}) \subseteq \partial g(\tilde{x})$ is not sufficient for $\tilde{x}$ to be a local minimizer.

Example 1.3.1 Let $f=g-h, g(x)=x^{4}+2, h(x)=x^{2}, \tilde{x}=0, S=[-0.5,0.5]$. Then $\partial h(0)=\{0\} \subseteq \partial g(0)=\{0\}$. But 0 is the maximum point of $f$ over $S$.

Example 1.3.1 shows that, in some cases, $\partial h(\tilde{x}) \subseteq \partial g(\tilde{x})$ still holds when $\tilde{x}$ is the local maximizer of $g(x)-h(x)$. It's not easy to use the sub-differential in the optimality condition. Therefore, our algorithms will not use these types of sub-differential used optimality conditions.

\subsubsection{The Canonical Form of DC Problems}

Among all types of DC structures, the following form is called canonical:

$$
(C D C) \quad \min \{d x \mid x \in \Omega \backslash \operatorname{int} C\}
$$

where $\Omega \subseteq \mathbb{R}^{n}$ and $C \subseteq \mathbb{R}^{n}$ are full-dimensional closed convex sets, $d \in \mathbb{R}^{n}$, and $d x$ denotes the scalar product between $d$ and the vector of variables $x \in \mathbb{R}^{n}$. 
Lemma 1.3.1 [113, Corollary 1] Any DC optimization problem can be transformed into form $(C D C)$.

Proof. Consider an arbitrary DC optimization problem

$$
\begin{gathered}
\min f_{0}(x) \\
f_{i}(x) \leq 0 \quad \text { s.t. } \quad i=1, \ldots, m .
\end{gathered}
$$

By introducing an additional variable $t \in \mathbb{R}$ such that $f_{0}(x) \leq t,(1.15)$ is equivalent to the following problem:

$$
\min \quad t \text { s.t. } f(x, t) \leq 0 \text {, }
$$

where

$$
f(x, t)=\max \left\{f_{0}(x)-t, \max \left\{f_{i}(x) \mid i=1,2, \ldots, m\right\}\right\} .
$$

By Proposition 1.1.2, the function $f(x, t)$ is still DC. Select convex functions $g(x, t)$ and $h(x, t)$ such that $f(x, t)=g(x, t)-h(x, t)$. By introducing another variable $\alpha \in \mathbb{R}$, the problem (1.16) can be rewritten as:

$$
\min t \text { s.t. } g(x, t)-\alpha \leq 0, h(x, t)-\alpha \geq 0 \text {. }
$$

Therefore, choose $d=(0, \ldots, 0,1), \Omega=\left\{(x, t, \alpha) \in \mathbb{R}^{n+2} \mid g(x, t)-\alpha \leq 0\right\}$ and $C=\left\{(x, t, \alpha) \in \mathbb{R}^{n+2} \mid h(x, t)-\alpha \leq 0\right\}$, the desired canonical form $(C D C)$ is obtained.

As any DC optimization problems can be transformed into this canonical form, any algorithm solving DC optimization problems of this form provides a unified way to solve all DC optimization problems. Moreover, this canonical form has a simple structure, that is a conventional convex minimization problem plus an additional reverse convex constraint. A deep understanding of this structure provides insight into the relevant properties and suggests efficient solution methods. Moreover, convex techniques have been developed rather maturely in the last decades, hence we can use the convex techniques to study the properties of convex and reverse convex constraints. Finally, its objective function is affine, which has many benefits, for instance its optimality conditions have a simple form. 
We recall a number of assumptions of $(C D C)$. The full-dimensionality assumption on $\Omega$ and $C$ is not restrictive; in fact, if $\Omega$ is not full-dimensional then the problem can be easily reformulated in the (affine) space generated by $\Omega$, while if $C$ is not full-dimensional then we have int $C=\emptyset$ and the problem is actually a convex minimization problem.

In order to avoid that $(C D C)$ could be reduced to a convex minimization problem, we also suppose that the set $C$ provides an essential constraint, i.e.

$$
\min \{d x \mid x \in \Omega\}<\min \{d x \mid x \in \Omega \backslash \operatorname{int} C\}
$$

Relying on an appropriate translation, assumption (1.17) can be equivalently stated thorough the following two conditions

$$
\begin{gathered}
0 \in \Omega \cap \operatorname{int} C, \\
d x>0 \quad \forall x \in \Omega \backslash \operatorname{int} C .
\end{gathered}
$$

Therefore, we assume that (1.18) and (1.19) hold. Notice that these assumptions guarantee that any feasible solution $x \in \Omega \backslash C$ provides a better feasible solution taking the unique intersection between the segment with 0 and $x$ as end points and the boundary of $C$, i.e. $x^{\prime} \in \partial C \cap(0, x)$ satisfies $d x^{\prime}<d x$. As a consequence, all optimal solutions to $(C D C)$ belong to the boundary of $C$. Relying upon an appropriate translation, we can suppose also that $0 \in$ int $\Omega$ without violating condition (1.19).

Definition 1.3.2 We say that the problem (CDC) is regular if

$$
\min \{d x \mid x \in \Omega \backslash \operatorname{int} C\}=\inf \{d x \mid x \in \Omega \backslash C\} .
$$

Furthermore,

$$
\gamma^{*}=\min \{d x \mid x \in \Omega \backslash \operatorname{int} C\}
$$

denotes the optimal value and

$$
D(\gamma)=\{x \in \Omega \mid d x \leq \gamma\}
$$

denotes the level set of $d x$ on $\Omega$. A value $\gamma$ is called feasible if there exists a feasible point $x \in \Omega \backslash \operatorname{int} C$ such that

$$
\gamma \geq d x \geq \gamma^{*}
$$


Proposition 1.3.2 If problem $(C D C)$ is regular, then a feasible value $\gamma$ is optimal if and only the following statements hold:

$$
\begin{gathered}
D(\gamma) \subseteq C, \\
C^{*} \subseteq[D(\gamma)]^{*} .
\end{gathered}
$$

Proof. $D(\gamma)$ and $C$ are both closed convex sets containing 0 , then conditions (1.22) and (1.23) are equivalent [113, Proposition 9]. When $\gamma$ is optimal, any $x^{1} \in D(\gamma) \backslash C$ will bring a feasible solution $x^{2} \in\left(0, x^{1}\right) \cap \partial C$ such that $d x^{2}<\gamma$. Therefore, $\gamma$ is not optimal, a contradiction.

Vice versa, suppose (1.22) or (1.23) holds. Regularity condition (1.20) guarantees the existence of $\left\{x^{k}\right\} \subseteq \Omega \backslash C$ such that $d x^{k} \downarrow \gamma^{*}$. Clearly, we have $D\left(d x^{k}\right) \backslash C \neq \emptyset$ which implies that $d x^{k} \geq \gamma$ for all $k$ and thus $\gamma^{*} \geq \gamma$. As $\gamma$ is also feasible, we get that $\gamma=\gamma^{*}$ and therefore $\gamma$ is optimal.

It should be noted that regularity condition (1.20) is only used to prove the sufficiency of conditions (1.22) and (1.23). Without (1.20), (1.22) and (1.23) are still necessary for the optimal value $\gamma$, but some feasible values that are not optimal may also satisfy them, as shown by the following example.

Example 1.3.2 Consider the (CDC) instance with $d=(0,1)$,

$$
\begin{gathered}
\Omega=\left\{\left(x_{1}, x_{2}\right) \mid-1 \leq x_{1} \leq 4,-2 \leq x_{2} \leq 4, x_{2} \geq-2-3 x_{1}\right\}, \\
C=\left\{\left(x_{1}, x_{2}\right) \mid-1 \leq x_{1} \leq 5,-2.5 \leq x_{2} \leq 6, x_{2} \leq x_{1}+3\right\} .
\end{gathered}
$$

Then $x^{*}=(-1,1)$ is the optimal point, but the point $x^{1}=(-1,2)$ also satisfies the optimality condition (1.22) and (1.23). In fact, the regularity condition (1.20) doesn't hold.

Example 1.3.2 shows that a DC optimization problem which does not satisfy the regularity condition (1.20) can not be solved by algorithms that check the optimality condition (1.22) or (1.23). By using these two optimality conditions, the following proposition provides a way to get the optimal objective value.

Proposition 1.3.3 If problem $(C D C)$ is regular, then solving $(C D C)$ amounts to searching for the value $\hat{\gamma}$ :

$$
\hat{\gamma}=\inf \{\gamma \mid D(\gamma) \backslash C \neq \emptyset\}=\sup \left\{\gamma \mid C^{*} \subseteq[D(\gamma)]^{*}\right\}
$$




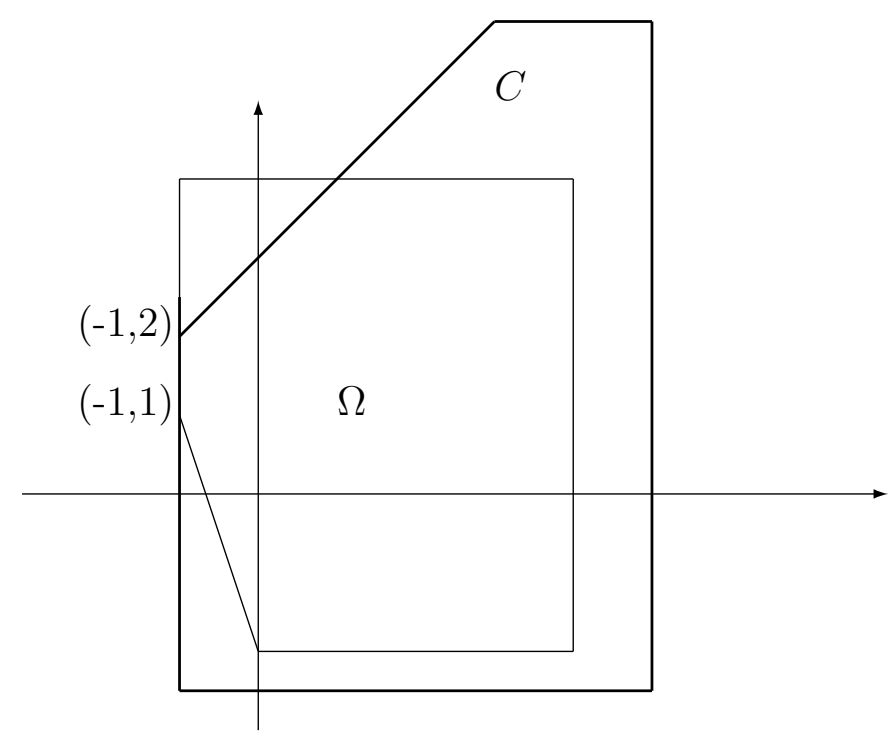

Figure 1.2: A not regular (CDC) instance in Example 1.3.2

Proof. It is trivial to prove the second inequality. Take any $\gamma$ such that $D(\gamma) \nsubseteq$ $C: D\left(\gamma^{*}\right) \subseteq C$ implies $\gamma^{*} \leq \gamma$ and thus $\gamma^{*} \leq \hat{\gamma}$. On the other side, regularity condition (1.20) guarantees a sequence $x^{k} \in \Omega \backslash C$ such that $d x^{k} \downarrow \gamma^{*}$. We also have $D\left(d x^{k}\right) \backslash C \neq \emptyset$ which implies $\hat{\gamma} \leq d x^{k}$ for all $k$ and therefore $\hat{\gamma} \leq \gamma^{*}$.

In order to guarantee the existence of optimal solutions, we may assume the boundedness of the level sets $D(\gamma)$ for the feasible values $\gamma$. Actually, such an assumption on the level sets is strictly related to the compactness of the reverse constraining set $C$ as the following result shows.

Lemma 1.3.2 Let $\gamma$ be a feasible value.

(i) If $C$ is compact, then so is $D(\gamma)$.

(ii) If $D(\gamma)$ is compact, then

$$
\gamma^{*}=\min \{d x \quad \mid x \in \Omega \backslash \operatorname{int} \hat{C}\}
$$

where $\hat{C}=C \cap B$ for any given compact set $B$ such that $D(\gamma) \subseteq \operatorname{int} B$. 
Proof. (i) Ab absurdo, suppose there exists a sequence $\left\{x^{k}\right\} \subseteq D(\gamma)$ such that $\left\|x^{k}\right\| \rightarrow+\infty$. Possibly taking a suitable subsequence, let $u=\lim _{k \rightarrow \infty} x^{k}\left\|x^{k}\right\|^{-1}$ : clearly $d u \leq 0$ and $u$ belongs to the recession cone of $\Omega$ [80, Theorem 8.2]. Since $0 \in \Omega$ and $C$ is bounded, there exists $\lambda>0$ such that $x^{0}=0+\lambda u \in \Omega \backslash C$. As $d x^{0} \leq 0$, assumption (1.19) is contradicted.

(ii) Let $\bar{\gamma}:=\min \{d x \mid x \in \Omega \backslash \operatorname{int} \hat{C}\}$. Since $\hat{C} \subseteq C$, then $\gamma^{*} \geq \bar{\gamma}$. Since $\gamma \geq \gamma^{*}$ the compactness of $D(\gamma)$ guarantees the existence of $\bar{x} \in \Omega \backslash$ int $\hat{C}$ such that $\bar{\gamma}=d \bar{x}$. As int $\hat{C}=\operatorname{int} C \cap \operatorname{int} B$ and $\bar{x} \in D(\gamma)$, then $\bar{x} \notin \operatorname{int} C: \bar{x}$ is feasible to $(C D C)$ and therefore $\gamma^{*} \leq \bar{\gamma}$.

Therefore, we assume that $C$ is compact throughout all the thesis. This ensures existence of an optimal solution $x^{*}$ of $(C D C)$, and therefore that $\gamma^{*}=$ $d x^{*}>0$ due to (1.19), a property that will turn out to be very useful.

The regularity condition (1.20) is strongly related to the existence of optimal solutions to $(C D C)$ with additional properties (see the Lemma below). Furthermore, regularity will be exploited to prove that stopping criteria with finite tolerance yield approximate optimal solutions.

Lemma 1.3.3 The regularity condition (1.20) holds if and only if $(C D C)$ has an optimal solution $x^{*} \in \partial(\Omega \backslash C)$.

Proof. Given any optimal solution $x^{*} \in \partial(\Omega \backslash C)$, there exists a sequence $\left\{x^{k}\right\}$ such that $x^{k} \in \Omega \backslash C$ and $x^{k} \rightarrow x^{*}$; hence

$$
\inf \{d x \mid x \in \Omega \backslash C\} \leq \lim _{k \rightarrow \infty} d x^{k}=d x^{*}=\min \{d x \mid x \in \Omega \backslash \operatorname{int} C\} .
$$

As the reverse inequality always holds, the regularity condition (1.20) follows.

Vice versa, suppose the regularity condition (1.20) holds. Therefore, there exists a sequence $\left\{x^{k}\right\} \subseteq \Omega \backslash C$ such that $d x^{k} \downarrow \gamma^{*}$. By Lemma 1.3.2 the compactness of $C$ guarantees that $D(\gamma)$ is compact for $\gamma=d x^{1}$. Therefore, the sequence $\left\{x^{k}\right\}$ admits at least one cluster point $x^{*} \in \operatorname{cl}(\Omega \backslash C)$. Since $\Omega$ is closed and $x^{k} \notin C$ for all $k$, we have $x^{*} \in \Omega$ and $x^{*} \notin \operatorname{int} C$. This implies that $x^{*}$ is feasible and hence optimal as $d x^{*}=\gamma^{*}$. Since all optimal solutions belong to the boundary of $C$, then $x^{*} \notin \Omega \backslash C$ and therefore $x^{*} \in \partial(\Omega \backslash C)$. 
The constraint $x \notin$ int $C$ is the source of nonconvexity in problem $(C D C)$ and it is given just as a set relation. However, relying on the polarity between convex sets, we can express this nonconvex constraint in a different fashion. Exploiting bipolarity relations (see, for instance, [80]), it is easy to check that the assumption $0 \in$ int $C$ ensures that $x \notin$ int $C$ if and only if $w x \geq 1$ for some $w \in C^{*}$. Therefore, problem $(C D C)$ can be equivalently formulated as

$$
\min \left\{d x \mid x \in \Omega, w \in C^{*}, w x \geq 1\right\}
$$

where polar variables $w$ have been introduced and the nonconvexity is given by the inequality constraint, which asks for some sort of reverse polar condition. Moreover, $x^{*} \in \Omega$ is an optimal solution of (CDC) if and only if there exists $w^{*} \in C^{*}$ s.t. $\left(x^{*}, w^{*}\right)$ is an optimal solution of (1.25). Also, the assumption $0 \in \operatorname{int} C$ ensures the compactness of $C^{*}$. The exploitation of polar variables will be an important tool to devise novel algorithms for $(C D C)$ through its reformulation (1.25).

Relying on bipolarity relations, the optimality condition (1.22) can be equivalently stated in a polar fashion as

$$
D(\gamma) \times C^{*} \subseteq\left\{(x, w) \in \mathbb{R}^{n} \times \mathbb{R}^{n} \mid w x \leq 1\right\}
$$

while the regularity condition (1.20) reads

$$
\min \left\{d x \mid x \in \Omega, w \in C^{*}, w x \geq 1\right\}=\inf \left\{d x \mid x \in \Omega, w \in C^{*}, w x>1\right\} .
$$

As an immediate consequence of (1.26), any optimal solution $\left(x^{*}, w^{*}\right)$ to $(1.25)$ satisfies both $x^{*} \in \partial C$ and $w^{*} x^{*}=1$.

In order to develop efficient solution methods for problem (CDC) and (1.25), it is important and necessary to have a deep understanding on the properties of the set of optimal solutions. At the beginning of this section, we have shown all optimal solution $\left(x^{*}, w^{*}\right)$ must satisfy $x^{*} \in \partial C$. Here we will introduce some other useful properties of $\left(x^{*}, w^{*}\right)$.

Definition 1.3.3 $\delta(x \mid C)$ is called the indicator function of $C \in \mathbb{R}^{n}$ where $\delta(x)=0$ if $x \in C$ and $\delta(x)=+\infty$ if $x \notin C$. 
Lemma 1.3.4 Given two closed convex sets $\Omega$, D. Let $d \in \mathbb{R}^{n}$ and $\theta(x)=$ $d x+\delta(x \mid \Omega)$. If $x^{*} \in$ int $\Omega$ is the minimum point of $\theta(x)$ on the set $D$, then $d x^{*} \leq d x$ for all $x \in D$, i.e. $-d$ is normal to the set $D$ at $x^{*}$.

Furthermore, if $D=\left\{x \in \mathbb{R}^{n} \mid w^{*} x \geq 1\right\}$ and $w^{*} x^{*}=1$, then $w^{*}=d / d x^{*}$.

Proof. Suppose ab absurdo that there exists $\hat{x} \in D$ such that $d \hat{x}<d x^{*}$, then the convexity of $D$ implies $\left[x^{*}, \hat{x}\right] \subseteq D$. It follows from $x^{*} \in$ int $\Omega$ that there exists a point $\tilde{x} \in\left(x^{*}, \hat{x}\right) \cap \Omega$ such that $d \tilde{x}<d x^{*}$. Therefore, we have both $\theta(\tilde{x})<\theta\left(x^{*}\right)$ and $\tilde{x} \in D$, in contradiction with the minimality of $x^{*}$.

Then, $-d\left(x-x^{*}\right) \leq 0$ for all $x \in\left\{x \in \mathbb{R}^{n} \mid w^{*}\left(x-x^{*}\right) \geq 0\right\}$. Let $y=x-x^{*}$, we have $-d y \leq 0$ for all $y$ s.t. $\left(-w^{*}\right) y \leq 0$. Then, there exists a $\lambda$ s.t. $\lambda w^{*}=d$ and $\lambda \geq 0$ by Farkas's Lemma. Therefore, there exists $\lambda^{\prime} \geq 0$ s.t. $d=\lambda^{\prime} w^{*}$. By $w^{*} x^{*}=1$ we have $\lambda^{\prime}=d x^{*}$ and thus $w^{*}=d / d x^{*}$.

Proposition 1.3.4 If $d \neq 0$ and there exists no optimal solution $\left(x^{*}, w^{*}\right)$ of (1.25) satisfying $x^{*} \in \partial \Omega$, then there exist $\gamma^{1}, \gamma^{2} \in \mathbb{R} \cup\{-\infty\} \cup\{+\infty\}$ s.t. $\gamma^{2} \geq \gamma^{1}$ and

$$
\Omega=\left\{x \in \mathbb{R}^{n} \mid \gamma^{1} \leq d x \leq \gamma^{2}\right\}
$$

Proof. Take any optimal solution $\left(x^{*}, w^{*}\right)$ and set $D=\left\{x \in \mathbb{R}^{n} \mid w^{*} x \geq 1\right\}$, then $\theta\left(x^{*}\right)=\min \{\theta(x) \mid x \in D\}$ where $\theta(x)=d x+\delta(x \mid \Omega)$. Since optimality implies $w^{*} x^{*}=1$, then Lemma 1.3.4 guarantees that $w^{*}=d / d x^{*}$.

If $\left\{x \in \mathbb{R}^{n} \mid d x=d x^{*}\right\} \cap \partial \Omega \neq \emptyset$, take any point $\hat{x} \in\left\{x \in \mathbb{R}^{n} \mid d x=d x^{*}\right\} \cap \partial \Omega$, we have $w^{*} \hat{x}=d \hat{x} / d x^{*}=1$ and hence $\left(\hat{x}, w^{*}\right)$ is an optimal solution, contradicting with the assumption. Therefore, $\left\{x \in \mathbb{R}^{n} \mid d x=d x^{*}\right\} \cap \partial \Omega=\emptyset$ and the hyperplane $\left\{x \in \mathbb{R}^{n} \mid d x=0\right\}$ is included in the recession cone of $\Omega$. Therefore, take $\gamma^{2}$ and $\gamma^{1}$ as the maximum and minimum value of $d x$ on $\Omega$, respectively, we get the conclusion.

These two propositions lead to the following property that has been given in Tuy's paper [113]. However, our proof is new since it uses the bipolarity properties from the above propositions.

Proposition 1.3.5 If $\partial \Omega \cap \partial C \neq \emptyset$, then there exists an optimal solution $\left(x^{*}, w^{*}\right)$ of (1.25) such that $x^{*} \in \partial \Omega \cap \partial C$. 
Proof. Consider the case that $x^{*} \in$ int $\Omega$. Let $\theta(x)=d x+\delta(x \mid \Omega)$ and $D=\left\{x \in \mathbb{R}^{n} \mid w^{*} x \geq 1\right\}$, we get that $x^{*}$ is a minimum point of $\theta(x)$ on $D$. Lemma 1.3.4 guarantees that $w^{*}=d / d x^{*}$.

$w^{*} \in C^{*}$ implies $d x / d x^{*} \leq 1$ for all $x \in C$. Take any point $\hat{x} \in \partial \Omega \cap \partial C$, then it must have $d \hat{x} \leq d x^{*}$. Moreover, $\hat{x} \in \partial C$ and boundedness of $C^{*}$ imply that there exists $\hat{w} \in C^{*}$ such that $\hat{w} \hat{x}=1$. Otherwise, we have $\max \left\{w \hat{x} \mid w \in C^{*}\right\}<1$, let $\rho(x \mid C)$ be the gauge function of $C$, then $\rho(\hat{x} \mid C)<1$ and thus $\hat{x} \notin \partial C[80$, Theorem 14.5]. Therefore, $(\hat{x}, \hat{w})$ is feasible and hence optimal.

\subsection{Literature Review}

The purpose of this section is to review the essential approaches and representative algorithms of concave minimization, reverse convex, concave minimization with an additional reverse convex constraint, general DC and canonical DC problems. All these problems have a close relation with the canonical DC and reverse polar problems.

All these problems display some special mathematical properties. The special mathematical properties and their applications have motivated the construction of numerous approaches for their solution. The scope of the presentation of the algorithmic methods is limited to those that use only deterministic methods; the readers interested in stochastic methods may look at Pardalos, Romeijn and Tuy [73] or Kan, Timmer [57, 58].

\subsubsection{Concave Minimization Problems}

Concave minimization problems are one of the most fundamental and intensively studied problems in the field of global optimization. This type of problems has a concave objective function and a closed convex feasible region. Sometimes it is also called convex maximization problem or multiextremal global optimization problem.

As it has been remarked in [9], Concave minimization problems have attracted such considerable attention and interest because they have the following characteristics: 
1. They display some interesting and attractive mathematical properties that make them more tractable than general global optimization problems.

2. They have a very broad range of direct applications.

3. Many global optimization problems can be transformed into concave minimization problems or are solvable via concave minimization.

4. Many algorithmic techniques used to solve concave minimization problems also play important roles in solving other types of global optimization problems.

Three fundamental classes of solution approaches can be distinguished. They are the enumerative, successive approximation, and successive partitioning approaches. It is sometimes possible to apply concave minimization methods directly in quasi-concave minimization problems. In the following paragraphs, we will discuss these three fundamental approaches for solving concave minimization problems and their representative algorithms. The algorithms for each approach are given in a historical order to make their evolution apparent.

Enumerative Methods Murty's algorithm [69] is the first enumerative approach which applies an extreme point ranking method. This algorithm devises a 'brute-force' procedure to find newly ranked extreme point. Murty's algorithm has many variants, such as Taha [88], Mckeown [64], Rubin [82], etc.

Another type of enumerative method is based on cutting planes, which was suggested by Horst and Tuy [46], and Cabot [17]. Their algorithms find a global optimal solution after a finite number of steps when certain conditions hold. Although the Tuy's cut or $\gamma$-valid cut is easy to construct, these cuts become shallower and shallower.

Then Majthay and Whinston suggested facial cuts, the advantage of this type of cuts is that facial cuts don't depend on the concavity of the objective function. Many variants of this algorithm have been proposed, such as Horst and Tuy [46], Fulop [27], etc.

The first cone covering algorithm for solving concave minimization problems was proposed by Tuy [99], which considered a special case where the constraint is polyhedral convex. His algorithm uses the Tuy's cuts to exclude parts of the feasible region, and applies a cone splitting procedure. However, his cone splitting algorithm is non-convergent, a counter example was shown by Zwart [129]. This algorithm was shown to cycle in some cases, some algorithms were given later for 
detecting the cycles, see [65]. Many variants of Tuy [99] have been proposed by Bali [4], Jacobsen [54], Horst and Tuy [46], Zwart [130]. This type of algorithms performs quite well even though they are not very theoretically satisfactory [9].

Successive Approximation Methods. The successive approximation approach for concave minimization problem constructs a sequence of improving approximations of the origin problem. These approximate problems are generally simpler to solve than the original problem. The approximate problems are usually solved sequentially until an exact or approximate global optimal solution to the original problem is found. The major successive approximation approaches are based on general outer approximation, outer approximation via collapsing polytopes and polyhedral annexation.

One of the more popular approaches to solving concave minimization problem by successive approximations is called outer approximation. The roots of the concept of using outer approximation via inequality cuts to globally solve concave minimization problem can be traced to early integer programming and convex programming methods developed by Gomory [30, 31], Cheney and Goldstein [18], Kelley [59], Veinott [124] and others. However, specific adaptations of this concept to concave minimization problem did not appear until the early 1980's.

Near the end of 1980's, Horst, Thoai and Tuy [50, 51] provided a general scheme and convergence theory for this type of algorithms. This general approach covers many algorithms provided by Thieu [94], Hoffman [53], Tuy [100], Horst and Tuy [46], etc.

Another approximation approach is to use collapsing polytopes to implement the outer approximation approach, a representative algorithm using collapsing polytopes was proposed by Falk and Hoffman [24]. This algorithm is finite, but it may enumerate all extreme points of the feasible region.

The polyhedral annexation approach constructs a sequence of inner approximations of the feasible set. This technique was firstly proposed by Tuy [99] and was improved by Tuy [106]. In each step, this approach finds a better extreme point or show no such point exists. Polyhedral Annexation is more efficient for solving problems with polyhedral feasible sets than outer approximation (Horst, Thoai and Tuy [51]). This is in part due to the number of extreme points in polyhedral annexation can be expected much smaller than those in outer ap- 
proximation.

More recently, concavity cut idea has been improved by exploiting representation cuts, which cuts a larger feasible portion than concavity cuts [77]. Then Porembski [79] proposed a new algorithm using representation and representation cuts, which resembles the conical partition algorithms. This algorithm finds a $\varepsilon$-global optimal solution in a finite number of steps. Besides, Porembski [78] proposed a cone adaptation idea that deepens concavity cuts, and then gave a finite exact cutting plane algorithm.

Successive Underestimation Another type of approach is called Successive Underestimation, which solves a special case where the feasible region is a bounded polyhedron. These approaches seek out a local solution which is used to eliminate this local solution from further consideration. The main disadvantage of this approach is that the linear subproblems typically grow in size at each iteration (Falk and Hoffman [23]). Falk and Hoffman [23] and Rosen [81]

proposed important algorithms of this type. In this approach, it is crucial to specify the under-estimators of the objective function.

One of the most popular approach for solving the concave minimization problems is the branch and bound method or successive partitioning method. Falk and Soland [25] proposed a branch and bound approach, which uses the convex envelopes and solves a sequence of subproblems where objective function is linear or convex. There are several partitioning rules, among which bisection is rather simple but it tends to slow convergence and fails to find the optimal solution in a finite number of steps [41]. Among the other rules, radial subdivision is relatively simple and performs better than the pure bisection. Ban's algorithm [5] subdivides each simplex into two subsimplices and is finite.

There are also many algorithms combining the above approaches. The interested readers may read [9].

\subsubsection{Concave Minimization Problems with a Reverse Con- vex Constraint}

This types of problems differs from the concave minimization problems by the presence of an additional reverse convex constraint. Thus they are more difficult 
to solve than the concave minimization problems. A common approach is transform this problem to a concave minimization problem, or solve it by solving a sequence of concave minimization subproblems.

Tuy [105] extended a property in Hillestad and Jacobsen [33] by proving that, the closure of the convex hull of the feasible set is a polyhedral set. Therefore, this type of global minimization problems can be theoretically reduced to a concave minimization problem. Then an extended version of the method by Ban [5] was given for solving this type of problems in Tuy [105].

Fulop [29] studied the lagrangian duality of the concave minimization problem with linear and reverse convex constraints and provided a finite method for solving this problem. This method is based on solving a sequence concave minimization problems.

\subsubsection{Reverse Convex Optimization Problems}

Reverse convex optimization plays an important role in global optimization. Generally, a reverse convex optimization problem has an convex objective function and the feasible set is the intersection of a convex set and a reverse convex set. Many methods have been published for solving this type of problems.

First, let's recall two properties on a special case where the feasible set is the intersection of a polyhedron and a reverse convex set. An important property of this problem is that it is sufficient to search for an optimal solution only on the 1-dimensional faces of the polyhedron. Fulop [28] used this property to propose a cutting plane method for solving the reverse convex optimization problem. Another important property of this problem is that the convex hull of bounded feasible region is a polytope and the solution lies at a vertex of this polytope, which has been exploited to propose an algorithm by Hillestad and Jacobsen [33]. Furthermore, Sen and Sherali [84] pointed out that, for certain special reverse convex sets, they construct a type of finite linear disjunction whose closed convex hull coincides with that of the special reverse convex set, which provides the capability of generating any facet cut.

One other approach it to solve reverse convex problems by solving a sequence of concave subproblems. One typical algorithm has been proposed by Tuy [114], where the outer approximation technique has been applied. Thach [91] presented 
a different approach, he converted the problem into the problem of finding the lexicographic minimum of the function vector over a compact convex set. This algorithm can be viewed as an improved version of the one in H. Tuy [114]. Horst, Phong and Thoai [45] showed that the reverse convex problems can be solved by a sequence of linear problems and line searches. Jacobsen and Khosrow [55] presented computational experience of an edge search algorithm for reverse convex problems. The algorithm is a depth first type algorithm and a variety of test problems is generated. Tuy and Nghia [120] proposed a representation method. This algorithm assumes that any feasible solution is the limit of a sequence of interior feasible points; Even when this condition doesn't hold, the algorithm can still find a $\varepsilon$-optimal solution in a finite number of steps.

Strekalovsky and Tsevendori [87] proposed an algorithm for solving the general reverse convex problem, which can be reduced to a $(C D C)$ problem. This algorithm use an equivalent optimality condition. The procedure of the Algorithm in [87] is similar to the Tuy's algorithm [102, 101]. In the global phase, the algorithm checks the optimality condition. If it doesn't hold, then choose a point that doesn't satisfy the optimality condition. Then finds a feasible point in the local phase and iterate. However, Tuan [98] shows that its implementation doesn't guarantee a correct solution and the optimality condition in this paper is not easier than the one in the other papers. Tseveendorj [97] presentes global optimality conditions for reverse convex problems. He also proves the convergence of an algorithm when the feasible domain is a polyhedron with an additional quadratic reverse convex constraint.

Moshirvaziri and Amouzegar [67] proposed a subdivision strategy on a cone containing the polyhedral space, which finds an upper bound and a lower bound and improves them at each iteration. When are the subdivisions are fathomed, the algorithm terminates and the optimal solution is found. Then they present a cutting plane method [68] within the framework of a branch-and-bound scheme. In this algorithm, the polytope associated with the linear constraints is partitioned. The algorithm systematically fathoms these portions and terminates when all the subdivisions have been fathomed.

Yamada, Tanino and Inuiguchi [128] presented an inner approximation method. The algorithm build a sequence of inner approximations and every accumulation point of the sequence of optimal solutions of the relaxed problem is an optimal 
solution of the original problem. An improvement of [128] was given in [127].

There are also some algorithms that are specialized for developing the local search methods of existing algorithms. Strekalovsky [86] proposed two versions of local search method for some existing algorithms, and convergence of his method with Tuy's algorithm is proved.

\subsubsection{General Constrained DC Optimization Problems}

In this subsection we review algorithms for constrained DC optimization problems, whose feasible region is a convex set and the objective function is a difference of two convex functions. This type of DC problems is called "general DC" in this thesis. DC optimization problems have a wide application in economics, engineering and other fields, see for instance the papers by Tuy [112] Konno [60], Blanquero and Carrizosa [13]. This problem covers the standard convex minimization problems and concave minimization problems, i.e. their objective function can be viewed as a difference of 0 and a convex function. In fact, any continuous global optimization problem on a compact set can be reformulated as a DC optimization problem [113]. DC optimization also has a close relationship with combinatorial optimization. Thus DC optimization technique can help us to handle a wide class of difficult global optimization problems. The deterministic DC optimization has a history of several decades and many algorithms were proposed for different types of DC problems. This section focuses only on the deterministic optimization algorithms.

Generally, in DC optimization problems, the regularity conditions is usually assumed, which requires that at least one optimal solution is a cluster point of interior feasible points. However, not all algorithms assume that regularity conditions holds, for instance Jeyakumar and Glover [56] established dual conditions characterizing global optimality of DC problems; as the optimality conditions are given in terms of $\varepsilon$-subdifferentials, the regularity condition is not assumed.

One approach is to transform the DC optimization problems to a concave minimization problem. In this way, many existing algorithms for solving concave minimization problems can be utilized. A typical algorithm is Tuy [102] under the assumption that the feasible region is a convex polyhedral set. This method converts the general DC problem to a concave minimization problem and applied 
the outer approximation method to the concave minimization problem. Liu and Papavassilopoulos [62] also transformed DC optimization problem into an equivalent concave minimization problem. They gave three parallel approaches on DC problems, then these approaches are applied and numerical tests are given.

Another approach is to explore the DC duality and local optimality conditions. In this type of algorithms, two sequences of candidate to primal and dual solutions are produced, which converge to the optimal solutions. P.D. Tao [89] proposed a sub-gradient algorithm for solving DC problems in 1986, which was improved and updated by Hoai An and Tao [39], [38] and [40]. Although these algorithms cannot guarantee the globality of computed solutions for general DC programs, they converge quite often to a global solution with a suitable starting point.

Branch and bound method is never missing. Horst, Phong, Thoai and Vries [52] proposed an algorithm for solving the bounded case of this problem. This algorithm combines the branch and bound technique, and the outer approximation technique. Its main advantage is that only linear subprograms and no univariate convex minimization problems have to be solved.

Covering algorithms have been also applied to DC optimization problems. Blanquero and Carrizosa [12] proposed an algorithm that is an generalization of Breiman and Cutler [16] proposed for solving DC optimization problems. These two algorithms split the feasible domain into polytopes and find local optimal points in the vertex points. The algorithm [12] yields not only more flexibility but also faster convergence.

\subsubsection{Canonical DC Optimization Problems}

Canonical DC optimization problems has a linear objective function, and its feasible region is an intersection of a convex set and a reverse convex set. All DC optimization problems can be reduced to the canonical DC form, and canonical DC form have a simple mathematical structure. Thus algorithms for solving canonical DC problems provide an unified treatment of DC problems. Many algorithms are given for solving this type of problems and they are either of branch and bound or outer approximation types.

Traditionally, these deterministic DC algorithms are classified into two classes: 
primal and dual.

The first cutting plane algorithm for canonical DC problems was proposed in [102] and [101]. In [101] Tuy introduced the canonical DC problem and showed how any DC problem can be reduced to this canonical form. His algorithm considers the bounded case and consists of two phases: a local phase and a global phase. At the end, Tuy proves that the algorithm terminates at an $\alpha$-optimal solution.

Since this algorithm requires a feasible solution at the beginning and it may not be trivial to find a feasible point, Tuy gave a modified algorithm in [112]. The framework of the new algorithm is same to the ones in [102] and [101], but there are also some differences, in particular, the local phase disappears. The start feasible value can be $+\infty$ when there is no available feasible point.

Thach [92] develops an outer approximation algorithm for finding feasible solutions to a system of nonlinear equations and inequalities. Then Phong, Tao and Hoai An presented an adaptation of Thach's algorithm for solving the Canonical DC problems. This method obtains satisfactory numerical results on the fuel mixture problem. A general approach was proposed by Tuy in [101], whose method covers many existing algorithms.

One other approach is to solve the canonical DC problems by solving a sequence of concave minimization subproblems. Nghia and Hieu [72] solved the canonical DC problems in this way. In the computational procedure, the mean value of the upper bound and lower bound is always checked and outer approximation technique is used.

One of the first Cut and Split algorithms was devised by Hillestad and Jacobsen [33] to solve reverse convex programs, i.e. canonical DC problems where $\Omega$ is a polyhedron. While this algorithm has solved a number of instances, its convergence property had not been proven until [107], where it is shown that if the algorithm performs the bisection step infinitely, then at least one cluster point of the algorithm is globally optimal.

Horst, Thoai and Benson [49] proposed the combined method firstly for concave minimization. An similar approach has been proposed in 1990, Horst, Phong and Thoai [48] showed that canonical DC optimization problems can be solved by a sequence of linear programs and univariate convex minimization problems. Although the procedure in this algorithm is similar to conical partition and outer 
approximation approach for the concave minimization problem, this algorithm is considerably different from those algorithms.

Not all existing algorithms can guarantee the convergence property. An attempt to solve canonical DC problems problem is given by Thoai [95]. The algorithm in [95] is a modified form of the ones in [102] and [101]. However, this algorithm as well as its modified form [48] are not guaranteed to converge [112]. Ben Saad and Jacobsen have also proposed cutting plane algorithms [6, 7], and their counter example was given later in [8].

In the class of dual methods, the polyhedral annexation algorithm and the dual CS/OA algorithm are the most famous. The former was proposed by Tuy [102] for concave minimization problems. Later it was extended to more general non-convex optimization problems [109] [111]. This algorithm uses the outer approximation procedure in the polar space. The Dual CS/OA method was firstly discussed by Tuy, Migdalas, Varbrand [123] for solving an equivalent problem of canonical DC problems. It is in fact a CS/OA algorithm in the polar space. 



\section{Chapter 2}

\section{Algorithms for Canonical DC Problems}

\section{$2.1 \quad$ Introduction}

As it have been stated in Chapter 1, nonconvex problems either have a natural formulation, or can be reformulated, as DC optimization problems. In turn, every DC optimization problem can be reduced, through standard transformations (see for instance [101]), to the canonical DC problem (CDC).

In this chapter, we consider the $(C D C)$ problem through its reformulation (1.25) and define a unified algorithmic framework for outer approximation type algorithms, which are based on an "oracle" for checking the global optimal conditions, and we study different sets of conditions which guarantee its convergence to (an approximated) optimal solution. As the oracle is the most computationally demanding part of the approach, we allow working with an approximated oracle which solves the related (nonconvex) optimization problem only approximately. Because of this, we provide an extensive analysis of approximate optimality conditions, which allow us to derive bounds on the quality of the obtained global solution. Our analysis identifies two main classes of approaches, which give rise to six different implementable algorithms, one of which is improved Tuy's algorithm [102], another one follows the same general procedure but takes an improved stopping criteria, and the other four are new and can't be reduced to the original cutting plane algorithm in [102] by Tuy. 
In Section 2.2 we propose a notion of approximate oracle, and define corresponding approximate optimality conditions, investigating the relationships between the exact optimal value and the approximate optimal values. In Section 2.3 a through convergence analysis is carried out for the "abstract" unified algorithmic framework, and then six different implementable algorithms are proposed which fit within the framework. Finally, in the last section the connections of these results with the existing algorithms in the literature are outlined.

\subsection{Approximate Optimality Conditions}

Given a feasible value $\gamma$, the optimality condition (1.22) or (1.26) should be checked in order to recognize whether or not $\gamma$ is the optimal value. Unfortunately, there is no known efficient way to check the inclusion between two sets. Yet, any exact algorithm for $(C D C)$ or (1.25) must eventually cope with this problem.

\subsubsection{Optimality Conditions and (Approximate) Oracles}

In order to make (1.22) or equivalently (1.26) more readily approachable, we consider the following "optimization version" of the optimality conditions:

$$
\max \left\{v z-1 \mid z \in D(\gamma), v \in C^{*}\right\}
$$

Obviously, (1.26) holds if and only if the optimal value $v\left(O C_{\gamma}\right)$ of $(2.1)$ is less or equal to 0 . Thus, the above problem provides a way for checking optimality of a given value $\gamma$. Since the objective function of (2.1) is not concave, there are no known efficient approaches for this problem as well. However, checking (1.26) through the optimization problem (2.1) has the advantage of making it easy to define a proper notion of approximate optimality conditions.

A first way of approximating problem $(2.1)$ is to replace $D(\gamma)$ and $C^{*}$ with two convex sets $S$ and $Q$, respectively, satisfying

$$
\begin{gathered}
C^{*} \subseteq Q \\
D(\gamma) \subseteq S .
\end{gathered}
$$


This is a standard step in cutting plane (outer approximation) approaches, where $S$ and $Q$ are chosen to be "easier" than the original sets (e.g. polyhedra with possibly few vertices or facets) and iteratively refined to become better and better approximations of $D(\gamma)$ and $C^{*}$ as needed. Hence, one considers the following relaxation of $(2.1)$

$$
\max \{v z-1 \mid z \in S, v \in Q\}
$$

whose optimal value $v\left(\overline{O C}_{\gamma}\right)$ provides an upper bound on $v\left(O C_{\gamma}\right)$; thus,

$$
v\left(\overline{O C}_{\gamma}\right) \leq 0
$$

is a convenient sufficient optimality condition for (1.25). If (2.5) does not hold, then either $\gamma$ is not the optimal value, or $S$ and $Q$ are not "good" approximations of $D(\gamma)$ and $C^{*}$, respectively. All the cutting plane algorithms presented in this work follow the same basic scheme: (2.4) is solved and its solution is used to improve $S$, or $Q$, or $\gamma$, in such a way to guarantee convergence of $\gamma$ to the optimal value. The focus of the research is on devising a number of different ways to achieve a convergent algorithm for (1.25) out of an "oracle" for (2.4). However, it is likely that in any such approach the solution of (2.4) is going to be the computational bottleneck; therefore, it makes sense to consider solving (2.4) only approximately.

Solving (2.4) approximately may actually mean two different things:

1. computing a "large enough" lower bound on $v\left(\overline{O C}_{\gamma}\right)$, i.e. finding a feasible solution $(\bar{z}, \bar{v})$ of $(2.4)$ "sufficiently close" to the optimal solution;

2. computing a "small enough" upper bound $l \geq v\left(\overline{O C}_{\gamma}\right)$.

Algorithmically, the two notions correspond to two entirely different classes of approaches: lower bounds are produced by heuristics computing feasible solutions, while upper bounds are produced by solving suitable relaxations of $\left(\overline{O C}_{\gamma}\right)$, e.g. replacing the non-concave objective function $v z$ with a suitable concave upper approximation. Exact algorithms combining the two can then be used to push the lower bound and the upper bound arbitrarily close together. However, for the sake of our approaches only one of the two bounds is needed at any given time. In fact, $v\left(\overline{O C}_{\gamma}\right)$ is either positive or non-positive. To establish that the 
first case holds amounts to finding a feasible solution $(\bar{z}, \bar{v})$ to $(2.4)$ such that $\bar{z} \bar{v}-1>0$, while for the second case one needs an upper bound $l \leq 0$.

This is the rationale behind our definition of an approximate oracle for (2.4). In our development we will assume availability of a procedure $\Theta$ which, given $S$, $Q, \gamma$, and two positive tolerances $\varepsilon$ and $\varepsilon^{\prime}$

- either produces an upper bound

$$
\varepsilon v\left(\overline{O C}_{\gamma}\right) \leq l \quad \text { such that } \quad l \leq \varepsilon^{\prime}
$$

- or produces a pair

$$
(\bar{z}, \bar{v}) \text { feasible for }(2.4) \quad \text { such that } \quad \bar{z} \bar{v}-1 \geq \varepsilon v\left(\overline{O C}_{\gamma}\right)>\varepsilon^{\prime}
$$

Clearly, (2.7) corresponds to a pretty weak requirement about the way in which (2.4) is solved: a solution, which is optimal only with fixed but arbitrary relative tolerance $\varepsilon>0$ and absolute tolerance $\varepsilon^{\prime}$, is required. Condition (2.6) allows the lower bound to be "small enough" but positive, rather than non-negative; this is taken as the stopping condition of the approach, and we will show that the positive tolerance allows for finite termination of the algorithms even when $\gamma$ is optimal. The drawback is that a feasible value $\gamma$ needn't be optimal when (2.6) holds: the next subsection is devoted to the study of the relationships between the "quality" of $\gamma$ and the tolerances $\varepsilon$ and $\varepsilon^{\prime}$.

\subsubsection{Approximate Optimality Conditions}

The stopping criterion (2.6) implies $v\left(O C_{\gamma}\right) \leq \varepsilon^{\prime} / \varepsilon$ : the tolerances provide the upper bound $\delta=\varepsilon^{\prime} / \varepsilon$ for the optimal value of (2.1). The values $\gamma$ for which this upper bound holds are strictly related to the following approximated problem

$$
\min \left\{d x \mid x \in \Omega, w \in C^{*}, w x \geq 1+\delta\right\}
$$

which is obtained by perturbing the right-hand side of the nonconvex constraint in (1.25). Our analysis does not require any regularity assumption on (2.8) and it is based on the following quantity

$$
\phi(\delta):=\inf \left\{d x \mid x \in \Omega, w \in C^{*}, w x>1+\delta\right\} .
$$


Obviously, $\phi(\delta)$ may be greater than the optimal value of (2.8). Anyway, the value function $\phi$ provides the right tool to disclose the connections between $\gamma$, (2.6) and (2.8).

Proposition 2.2.1 Let $\delta \geq 0$. Then, the following statements are equivalent:

(i) $v\left(O C_{\gamma}\right) \leq \delta$

(ii) $D(\gamma) \times C^{*} \subseteq\left\{(x, w) \in \mathbb{R}^{n} \times \mathbb{R}^{n} \mid w x \leq 1+\delta\right\} ;$

(iii) $\gamma \leq \phi(\delta)$

Proof. The equivalence between $(i)$ and $(i i)$ follows immediately from the definition of $v\left(O C_{\gamma}\right)$. Analogously, (ii) implies $(i i i)$ by the definition of $\phi(\delta)$.

Suppose (ii) does not hold: there exist $x \in D(\gamma)$ and $w \in C^{*}$ such that $w x>1+\delta$. Take any $t \in(0,1)$ large enough to have $w(t x)>1+\delta$. Since $0 \in \Omega$, the convexity of $\Omega$ implies $t x \in \Omega$; obviously $d(t x)<d x \leq \gamma$. Therefore, $(t x, w)$ guarantees $\phi(\delta)<\gamma$ contradicting (iii).

Considering the optimal value of (2.8) as $\gamma$ in Proposition 2.2.1, we get that (ii) is a necessary optimality condition for (2.8). Furthermore, if the problem is regular (i.e. $\phi(\delta)$ is actually the optimal value), it is also sufficient. Choosing $\delta=0$, the known optimality conditions for (1.25) follow too. Therefore, inclusion (ii) can be considered as an approximate optimality condition for (1.25). It is easy to check that $(i i)$ is equivalent to the inclusion $D(\gamma) \subseteq(1+\delta) C$ : perturbing the right-hand side of the nonconvex constraint in (1.25) corresponds to perturbing the reverse constraining set $C$ in $(C D C)$. As an immediate consequence of the proposition, we also have

$$
\phi(\delta)=\sup \left\{\gamma \mid D(\gamma) \times C^{*} \subseteq\left\{(x, w) \in \mathbb{R}^{n} \times \mathbb{R}^{n} \mid w x \leq 1+\delta\right\}\right\} .
$$

The stopping criterion $(i)$ guarantees approximate optimality and condition (iii) provides the adequate tool to evaluate the quality of the approximation. In fact, supposing (1.25) to be regular, i.e., $\gamma^{*}=\phi(0)$, we have that

$$
0 \leq \gamma-\gamma^{*} \leq \phi(\delta)-\gamma^{*}=\phi(\delta)-\phi(0)
$$

holds for any feasible value $\gamma$ which satisfies $(i)$. The following result guarantees that the approximation approaches the optimal value as $\delta$ goes to 0 . 
Proposition 2.2.2 The value function $\phi$ is right-continuous at 0, i.e.

$$
\lim _{\delta \downarrow 0} \phi(\delta)=\phi(0)
$$

Proof. Clearly $\phi$ is nonincreasing, that is $\phi\left(\delta^{1}\right) \geq \phi\left(\delta^{2}\right)$ whenever $\delta^{1} \geq \delta^{2} \geq 0$. As it is also bounded below by $\phi(0)$, there exist $\bar{\gamma}=\lim _{\delta \downarrow 0} \phi(\delta)$ and $\bar{\gamma} \geq \phi(0)$. Since $\bar{\gamma} \leq \phi(\delta)$ for any $\delta>0$, Proposition 2.2.1 implies $v\left(O C_{\bar{\gamma}}\right) \leq \delta$ for any $\delta>0$. Since $v\left(O C_{\bar{\gamma}}\right)$ does not depend upon $\delta$, we get $v\left(O C_{\bar{\gamma}}\right) \leq 0$. Therefore, Proposition 2.2.1 guarantees $\bar{\gamma} \leq \phi(0)$.

Although the approximation always converges to the optimal value, the rate of convergence may be less than linear as the following example shows.

Example 2.2.1 Consider (2.8) with $n=2, d=(-1,2), \Omega=[-2,0] \times[0,+\infty)$ and $C=\left\{x \in \mathbb{R}^{2} \mid x_{1}^{2}+x_{2}^{2} \leq 4\right\}$. It is easy to check that (2.8) is regular for any $\delta \geq 0$ and that $\left(x^{*}, w^{*}\right)=\left(\left(-2,2 \sqrt{(1+\delta)^{2}-1}\right),(-1 / 2,0)\right)$ is an optimal solution to $(2.8)$ for $\delta \leq 1 / 4$. Therefore, we have $\phi(\delta)=4 \sqrt{(1+\delta)^{2}-1}+2$ and

$$
\lim _{\delta \downarrow 0}[\phi(\delta)-\phi(0)] / \delta=\lim _{\delta \downarrow 0} 4 \sqrt{1+2 / \delta}=+\infty .
$$

Thus regularity is not enough to achieve a linear rate of convergence. Additional assumptions on the problem are needed: the existence of an optimal solution with some particular properties guarantees the Lipschitz behavior of $\phi$.

Proposition 2.2.3 If there exists an optimal solution $\left(x^{*}, w^{*}\right)$ to (1.25) such that

$$
\left\{x^{*}+\lambda u \mid \lambda>0\right\} \cap \Omega \neq \emptyset \text { and } w^{*} u>0
$$

for some direction $u \in \mathbb{R}^{n}$, then the value function $\phi$ is locally Lipschitz at 0, i.e. there exist $L>0$ and $\bar{\delta}>0$ such that

$$
\phi(\delta)-\phi(0) \leq L \delta \quad \forall \delta \in[0, \bar{\delta}]
$$

Proof. Let $\bar{\lambda}>0$ be such that $x^{*}+\bar{\lambda} u \in \Omega$; the convexity of $\Omega$ implies $x(\lambda):=$ $x^{*}+\lambda u \in \Omega$ for any $\lambda \in[0, \bar{\lambda}]$; furthermore, $w^{*}\left(x^{*}+\lambda u\right)=1+\lambda w^{*} u>1$ if $\lambda>0$. Thus, the sequence $\left(x(\lambda), w^{*}\right)$ shows that the regularity condition (1.27) holds. Therefore, we have $\phi(0)=d x^{*}$. 
Chosen $\bar{\delta}:=\left(w^{*} u / 2\right) \bar{\lambda}$, let us consider $y(\delta):=x\left(2 \delta / w^{*} u\right)$ for any $\delta \in(0, \bar{\delta}]$ : we have $y(\delta) \in \Omega$ and

$$
w^{*} y(\delta)=w^{*} x^{*}+\left(2 \delta / w^{*} u\right) w^{*} u=1+2 \delta>1+\delta,
$$

where the last equality holds since optimality implies $w^{*} x^{*}=1$. Therefore, $\left(y(\delta), w^{*}\right)$ provides an upper bound for $\phi(\delta)$, i.e. $\phi(\delta) \leq d y(\delta)$. Finally, we get

$$
\phi(\delta)-\phi(0) \leq d y(\delta)-d x^{*}=\left(2 d u / w^{*} u\right) \delta
$$

Though regularity has not been explicitly required for (1.25), the assumption on the optimal solution implies it. A geometric view of this assumption can be achieved relying on the (Bouligand) tangent cone of $C$ at $x^{*}$, namely the set

$$
T(C, x):=\left\{u \in \mathbb{R}^{n} \mid \exists t_{n} \downarrow 0, u_{n} \rightarrow u \text { s.t. } x+t_{n} u_{n} \in C\right\},
$$

and its following characterization.

Lemma 2.2.1 Let $x^{*} \in \partial C$. Then, the following statements are equivalent:

(i) $u \in T\left(C, x^{*}\right)$;

(ii) $w u \leq 0$ for all $w \in C^{*}$ such that $w x^{*}=1$.

Proof. Take any $u \in T\left(C, x^{*}\right)$ : there exist $t_{n} \downarrow 0$ and $u_{n} \rightarrow u$ such that $x^{*}+t_{n} u_{n} \in C$. Therefore, we have $w\left(x^{*}+t_{n} u_{n}\right) \leq 1$ for any $w \in C^{*}$. If $w x^{*}=1$, we get $w u_{n} \leq 0$ and taking the limit $w u \leq 0$.

Vice versa, suppose $u$ satisfies $(i i)$ but $u \notin T\left(C, x^{*}\right)$. Since the tangent cone is a closed set, there exists $\varepsilon>0$ such that $\hat{u}=u-\varepsilon x^{*} \notin T\left(C, x^{*}\right)$. Consider any $t_{n} \downarrow 0$ and $u_{n} \rightarrow \hat{u}$ such that $x^{*}+t_{n} u_{n} \notin C$. Therefore, there exist $w_{n} \in C^{*}$ such that $w_{n}\left(x^{*}+t_{n} u_{n}\right)>1$. Assumption (1.18) implies that $C^{*}$ is compact (see, for instance, [80, Corollary 14.5.1]). Thus, we can suppose $w_{n} \rightarrow \bar{w}$ for some $\bar{w} \in C^{*}$. Taking the limit in the above inequality, we get $\bar{w} x^{*} \geq 1$ and therefore $\bar{w} x^{*}=1$. Since $t_{n} w_{n} u_{n}>1-w_{n} x^{*} \geq 0$, we also get $\bar{w} \hat{u} \geq 0$. The assumption on $u$ guarantees also $\bar{w} u \leq 0$. Therefore, we get the contradiction $0 \leq \bar{w} \hat{u}=\bar{w}\left(u-\varepsilon x^{*}\right) \leq-\varepsilon$.

The following characterization allows to formulate the assumption of Proposition 2.2.3 in a geometric fashion. 
Proposition 2.2.4 Let $x^{*} \in \partial C$. Then, the following statements are equivalent:

(i) there exist $w^{*} \in C^{*}$ and $u \in \mathbb{R}^{n}$ such that $w^{*} x^{*}=1$ and (2.10) holds;

(ii) $T\left(\Omega, x^{*}\right) \nsubseteq T\left(C, x^{*}\right)$.

Proof. Suppose $(i i)$ does not hold and take any $w^{*} \in C^{*}$ and $u \in \mathbb{R}^{n}$ such that $w^{*} x^{*}=1$ and $x^{*}+\bar{\lambda} u \in \Omega$ for some $\bar{\lambda}>0$. The convexity of $\Omega$ implies $\Omega \subseteq x^{*}+T\left(\Omega, x^{*}\right)$ and therefore $\bar{\lambda} u \in T\left(\Omega, x^{*}\right) \subseteq T\left(C, x^{*}\right)$. By Lemma 2.2.1 we get $w^{*} u \leq 0$ : hence $(i)$ does not hold.

Vice versa, take any $u \in T\left(\Omega, x^{*}\right) \backslash T\left(C, x^{*}\right)$. Lemma 2.2.1 implies that there exists $w^{*} \in C^{*}$ such that $w^{*} x^{*}=1$ and $w^{*} u>0$. As $u \in T\left(\Omega, x^{*}\right)$, there exist $t_{n} \downarrow 0$ and $u_{n} \rightarrow u$ such that $x^{*}+t_{n} u_{n} \in \Omega$; if $n$ is large enough, we also have $w^{*} u_{n}>0$. Thus, $w^{*}$ and $u_{n}$ satisfy $(2.10)$.

It is worth to note that $(i i)$ depends upon $x^{*}$ only. Indeed, the original formulation of the Canonical DC problem does not have polar variables. Anyway, $x^{*}$ is an optimal solution to $(C D C)$ if and only if $\left(x^{*}, w^{*}\right)$ is an optimal solution to (1.25) for any $w^{*} \in C^{*}$ such that $w^{*} x^{*}=1$. As a consequence, Propositions 2.2.3 and 2.2.4 lead to the main result of the section.

Theorem 2.2.1 If there exists an optimal solution $\left(x^{*}, w^{*}\right)$ to (1.25) such that $T\left(\Omega, x^{*}\right) \nsubseteq T\left(C, x^{*}\right)$, then $\phi$ is locally Lipschitz at 0 .

The assumption on the tangent cones can be considered as a strong regularity condition. In fact, it implies regularity but they are not equivalent, as the problem of Example 2.2.1 shows for $\delta=0$. Anyway, when $C$ is a polyhedron, strong regularity collapses to regularity.

Theorem 2.2.2 Suppose $C$ is a polyhedron. Then, (1.25) is regular if and only if there exists an optimal solution $\left(x^{*}, w^{*}\right)$ to (1.25) such that $T\left(\Omega, x^{*}\right) \nsubseteq T\left(C, x^{*}\right)$.

Proof. Suppose (1.25) is regular: Lemma 1.3.3 implies the existence of an optimal solution $\left(x^{*}, w^{*}\right)$ to $(1.25)$ such that $x^{*} \in \partial(\Omega \backslash C)$. Suppose $T\left(\Omega, x^{*}\right) \subseteq$ $T\left(C, x^{*}\right)$. Since $C$ is a polyhedron, there exists $\varepsilon>0$ such that

$$
\left[x^{*}+T\left(C, x^{*}\right)\right] \cap B\left(x^{*}, \varepsilon\right)=C \cap B\left(x^{*}, \varepsilon\right) .
$$


Since the convexity of $\Omega$ implies $\Omega \subseteq x^{*}+T\left(\Omega, x^{*}\right)$, we have

$$
\Omega \cap B\left(x^{*}, \varepsilon\right) \subseteq C \cap B\left(x^{*}, \varepsilon\right)
$$

in contradiction with $x^{*} \in \partial(\Omega \backslash C)$. The if part follows from Proposition 2.2.4 and the proof of Proposition 2.2.3.

Corollary 2.2.1 Suppose $C$ is a polyhedron. If (1.25) is regular, then $\phi$ is locally Lipschitz at 0 .

\subsection{Conditions and Algorithms}

In this section we present several algorithms which approximately solve the problem $(C D C)$ through its polar reformulation (1.25) if an approximated oracle $\Theta$ is available. We first establish a hierarchy of abstract conditions ensuring convergence; then, for each set of conditions we propose actual implementatable procedures which realize it.

\subsubsection{General Convergence Conditions}

All the algorithms will follow the generic cutting plane scheme sketched in the previous section. More in details, a non increasing sequence of feasible values $\left\{\gamma^{k}\right\}$ is produced, and the oracle $\Theta$ is called for each $\gamma^{k}$, thereby producing either a value $l^{k}$ such that condition (2.6) holds, or points $z^{k}$ and $v^{k}$ such that (2.7) are satisfied. By repeatedly calling the oracle, we can construct a procedure which either proves that $\gamma^{k}$ satisfies condition (2.6) or produces a better feasible value $\gamma^{k+1}<\gamma^{k}$. In the latter case, $\gamma^{k+1}$ is associated to (produced by) points $x^{k}$ and $w^{k}$ such that

$$
x^{k} \in C, \quad w^{k} \in C^{*}, \quad w^{k} x^{k}=1
$$

which implies also $\left(x^{k}, w^{k}\right) \in \partial C \times \partial C^{*}$ (see [80, Proposition 13.1]). The rationale for (2.11) is that any optimal solution must satisfy these conditions.

It must be stressed that the above conditions do not require $x \in \Omega$; and therefore $\left(x^{k}, w^{k}\right)$ may be unfeasible for the polar reformulation (1.25). Anyway, 
(1.25) can be equivalently stated as

$$
\min \left\{\zeta(w) \mid w \in C^{*}\right\}
$$

where

$$
\zeta(w)=\min \{\theta(x) \mid w x \geq 1\} .
$$

and

$$
\theta(x)= \begin{cases}d x & \text { if } x \in \Omega \\ +\infty & \text { otherwise }\end{cases}
$$

Therefore, the polar variable $w^{k}$ is always feasible for (2.12), though it may be $\theta\left(x^{k}\right)=+\infty$. Since $\zeta(w) \leq \theta(x)$ for all pairs $(x, w)$ satisfying $(2.11)$, we can choose $\gamma^{k+1}=\zeta\left(w^{k}\right)$ whenever $x^{k} \notin \Omega$. As $\zeta\left(w^{k}\right)$ is the optimal value of a convex problem, it can be assumed to be efficiently computable. Moreover, if $\gamma^{k+1}$ turns out to be optimal, then $w^{k}$ is the "polar part" of an optimal solution; in fact any

$$
\bar{x} \in \operatorname{argmin}\left\{d x \mid x \in \Omega, w^{k} x \geq 1\right\}
$$

provides the complementary $x$ part of the optimal solution.

Thus, a given pair $\left(x^{k}, w^{k}\right)$ can provide two (potentially) different feasible values: $\theta\left(x^{k}\right)$ which is essentially costless to compute but may be infinite, and $\zeta\left(w^{k}\right)$ whose computation requires the solution of a convex program. In general one may want to avoid the computation of $\zeta\left(w^{k}\right)$ unless it is strictly necessary; to allow a general treatment we will in the following indicate with $\gamma(x, w)$ a function taking a pair $(x, w)$ satisfying $(2.11)$ and producing a feasible value. Which of the two possible implementations $(\theta$ and $\zeta$ ) is required will be discussed in the context of each implementable algorithm.

With the above notation, we can introduce the prototype of our algorithms.

\section{Algorithm 1 Prototype Algorithm}

0. $\gamma^{1}=+\infty ; k=1$;

1. If optimality condition (1.22) holds, then $\gamma^{k}$ is the optimal value: stop;

2. Select $\left(x^{k}, w^{k}\right)$ satisfying (2.11) such that $\gamma^{k+1}=\gamma\left(x^{k}, w^{k}\right)<\gamma^{k}$;

set $k=k+1$; goto 1 .

Clearly, if at Step 0 (initialization) some feasible pair $\left(x^{0}, w^{0}\right)$ is known, one can alternatively set $\gamma^{1}=\gamma\left(x^{0}, w^{0}\right)$. An important feature of Algorithm 1 is that 
$\left\{\gamma^{k}\right\}$ is a decreasing sequence bounded below:

$$
0 \leq \lim _{k \rightarrow \infty} \gamma^{k}=\gamma^{\infty}<\cdots<\gamma^{k+1}<\gamma^{k}<\cdots<\gamma^{1}
$$

Therefore, $\left\{D\left(\gamma^{k}\right)\right\}$ is a "non-increasing" sequence, i.e.

$$
D\left(\gamma^{\infty}\right) \subseteq \cdots \subseteq D\left(\gamma^{k+1}\right) \subseteq D\left(\gamma^{k}\right) \subseteq \cdots \subseteq D\left(\gamma^{1}\right)
$$

Obviously, Algorithm 1 is too general to deduce any meaningful property; some more has to be said:

1. how exactly optimality condition (1.22) is checked,

2. how $\left(x^{k}, w^{k}\right)$ such that $\gamma\left(x^{k}, w^{k}\right)<\gamma^{k}$ is selected once one knows that (1.22) is not fulfilled.

The two points are strictly interwoven: finding $\left(x^{k}, w^{k}\right)$ such that $\gamma\left(x^{k}, w^{k}\right)<\gamma^{k}$ immediately proves that $\gamma^{k}$ is not optimal. Vice versa, assume that we have any constructive procedure that produces a point $z^{k} \in D\left(\gamma^{k}\right) \backslash C$ when $\gamma^{k}$ is not optimal: there exists $w^{k} \in C^{*}$ such that $w^{k} z^{k}>1$ and $x^{k}=\left(w^{k} z^{k}\right)^{-1} z^{k}$ satisfying both $x^{k} \in D\left(\gamma^{k}\right)$ and $\gamma\left(x^{k}, w^{k}\right) \leq d x^{k}<d z^{k} \leq \gamma^{k}$.

Then, a first question is if such a method provides a convergent algorithm; not surprisingly, without further qualification the answer is negative.

Example 2.3.1 Consider (1.25) with $n=2, d=(0,1)$, and the sets

$$
\Omega=\left\{x \in \mathbb{R}^{2} \quad \mid-1.8 \leq x_{1} \leq 1.96, x_{2} \geq 0\right\}, \quad C=\left\{x \in \mathbb{R}^{2} \quad \mid x_{1}^{2}+x_{2}^{2} \leq 4\right\}
$$

therefore, we have

$$
C^{*}=\left\{\left(x_{1}, x_{2}\right) \in \mathbb{R}^{2} \mid x_{1}^{2}+x_{2}^{2} \leq 1 / 4\right\}
$$

Starting from any value $\gamma^{1}>0.87$ and applying the above procedure, we can find the sequences

$$
z^{k}=\left(-1.8, \gamma^{k-1}\right), \quad x^{k}=\frac{2 z^{k}}{\sqrt{1.8^{2}+\left(\gamma^{k-1}\right)^{2}}}, \quad w^{k}=\frac{z^{k}}{2 \sqrt{1.8^{2}+\left(\gamma^{k-1}\right)^{2}}}
$$

which lead to a non-optimal solution $x^{\infty}=(-1.8,0.87)$ and $w^{\infty}=(1 / 4) x^{\infty}$, whereas the optimal solution is $\left(x^{*}, w^{*}\right)=((1.96,0.4),(1.96,0.4) / 4)$. 
Thus, some care is needed in choosing the sequence $w^{k}$ in Algorithm 1, as well as the accompanying sequences $z^{k}$ and $x^{k}$ if the mechanism illustrated above is to be used. Actually, our "more implementable" approximate optimality condition based on (2.1) indicates that a fourth sequence $v^{k}$, which "is to $w^{k}$ what $z^{k}$ is to $x^{k}$, should be taken into account as well. In fact, we propose the following general assumptions under which convergence can be proved:

$$
\begin{gathered}
v^{k} z^{k}-1 \geq \varepsilon \max \left\{v z-1 \mid(z, v) \in D\left(\gamma^{k}\right) \times C^{*}\right\} \\
\liminf _{k \rightarrow \infty} v^{k} z^{k} \leq 1
\end{gathered}
$$

where $\varepsilon \in(0,1)$. Condition (2.16) basically says that $v^{k}$ and $z^{k}$ must be produced by some process attempting to solve non-convex problem (2.1) for $\gamma=\gamma^{k}$, i.e. the problem $\left(O C_{\gamma^{k}}\right)$, although the process may be "terminated early" due to the optimality tolerance $\varepsilon$. Condition (2.17) rather requires the two sequences to be asymptotically jointly feasible, and, as we will see, there are several different implementable ways for ensuring that this holds. Anyway, as far as abstract conditions go, (2.16) and (2.17) are sufficient to guarantee convergence to the optimal value.

Proposition 2.3.1 If conditions (2.16) and (2.17) hold, then the sequence of feasible values $\left\{\gamma^{k}\right\}$ in Algorithm 1 converges to the optimal value $\gamma^{*}$.

Proof. Since each $\gamma^{k}$ is a feasible value, we have $\gamma^{*} \leq \gamma^{\infty}$, i.e. $\gamma^{\infty}$ is a feasible value, too. Hence, (2.16) implies that

$$
v^{k} z^{k}-1 \geq \varepsilon \max \left\{v z-1 \mid(z, v) \in D\left(\gamma^{\infty}\right) \times C^{*}\right\}
$$

for all $k$. It follows from (2.17) that

$$
\max \left\{v z-1 \mid(z, v) \in D\left(\gamma^{\infty}\right) \times C^{*}\right\} \leq 0
$$

i.e., $\gamma^{\infty} \leq \gamma^{*}$ and thus $\gamma^{\infty}=\gamma^{*}$.

When developing a "concrete" algorithm for (CDC), the abstract condition (2.17) should not be directly imposed on the sequences $\left\{z^{k}\right\}$ and $\left\{v^{k}\right\}$. In fact, these are the results of a "complex" optimization process, i.e. approximately solving (2.1), upon which we want to impose as few conditions as possible, in 
order to leave as much freedom as possible to different implementations of this critical task. Therefore, we seek alternative ways for obtaining condition (2.17). One possibility is to rely on sequences of points $x^{k}$ and $w^{k}$, which satisfy one of these pairs of conditions:

$$
\begin{aligned}
& \left\{\begin{array}{l}
v^{k} z^{k} \leq v^{k} x^{k}+\sigma_{1}^{k} \\
v^{k} x^{k} \leq 1+\sigma_{2}^{k}
\end{array}\right. \\
& \left\{\begin{array}{l}
v^{k} z^{k} \leq w^{k} z^{k}+\sigma_{3}^{k} \\
w^{k} z^{k} \leq 1+\sigma_{4}^{k}
\end{array}\right.
\end{aligned}
$$

$\left\{\sigma_{1}^{k}\right\},\left\{\sigma_{2}^{k}\right\},\left\{\sigma_{3}^{k}\right\}$ and $\left\{\sigma_{4}^{k}\right\}$ are four positive sequences converging to zero. Both pairs of conditions clearly imply (2.17).

Lemma 2.3.1 If either (2.18) or (2.19) hold, then (2.17) holds.

Proof. Joining (2.18a) and (2.18b) we get $v^{k} z^{k} \leq 1+\sigma_{1}^{k}+\sigma_{2}^{k}$, and therefore (2.17) follows. The proof of (2.19) is analogous.

Therefore, we can define the two sets of conditions which, separately, guarantee convergence of Algorithm 1:

$$
B_{1} \equiv(2.16) \text { and }(2.18) \quad B_{2} \equiv(2.16) \text { and }(2.19)
$$

Though they look highly symmetric to each other but, we will show that $B_{1}$ and $B_{2}$ are by no means equivalent. In fact, we will propose several different sets of conditions (in particular, four for $B_{1}$ and two for $B_{2}$ ) which imply one of them, and develop implementable sub-procedures that attain these conditions, thereby defining six different implementable algorithms.

\subsubsection{The Outer Approximation Machinery}

As addressed before, one important way to make (2.1) more tractable is to replace $D(\gamma)$ and $C^{*}$ with two "simpler" convex sets $Q$ and $S$ such that $C^{*} \subseteq Q$ and $D(\gamma) \subseteq S$. Of course, this requires some appropriate machinery to update $S$ and $Q$ in order to make them "good enough" approximations of $D(\gamma)$ and $C^{*}$. Convexity of both sets allows us to rely on cutting procedures based on standard 
separation tools. In fact, the result below follows readily from the general Basic Outer Approximation Theorem [46, Theorem II.1].

Theorem 2.3.1 Let $r$ be a convex function such that $R=\left\{x \in \mathbb{R}^{n} \mid r(x) \leq 0\right\}$ satisfies $0 \in \operatorname{int} R$. Let $\left\{R^{k}\right\}$ be a sequence of convex sets and $\left\{x^{k}\right\}$ a sequence of points which satisfy the following conditions

1. $x^{k} \in R^{k} \backslash R$;

2. $R^{k+1}=R^{k} \cap\left\{x \in \mathbb{R}^{n} \mid p^{k}\left(x-y^{k}\right)+r\left(y^{k}\right) \leq 0\right\}$, where $p^{k} \in \partial r\left(y^{k}\right)$ for some $y^{k} \in\left[0, x^{k}\right) \backslash \operatorname{int} R$.

Then, any cluster point $\bar{x}$ of the sequence $\left\{x^{k}\right\}$ belongs to $\partial R$.

Theorem 2.3.1 suggests the standard cutting-plane procedure described in Sub-procedure 2: it takes a "simple" representation $S$, typically a polyhedron, of the convex set $R$ and a point $x$ which proves the two are different; it "improves" $S$ to a representation of $R$ which does not contain $x$, and still is a polyhedron if $S$ is, by intersecting $S$ with a separating hyperplane which cuts off $x$ but no point in $R$. Due to Theorem 2.3.1, iterating this process leads, in the limit, to a point in $R$; in other words, $S$ becomes an "arbitrarily close" representation of $R$ near a cluster point.

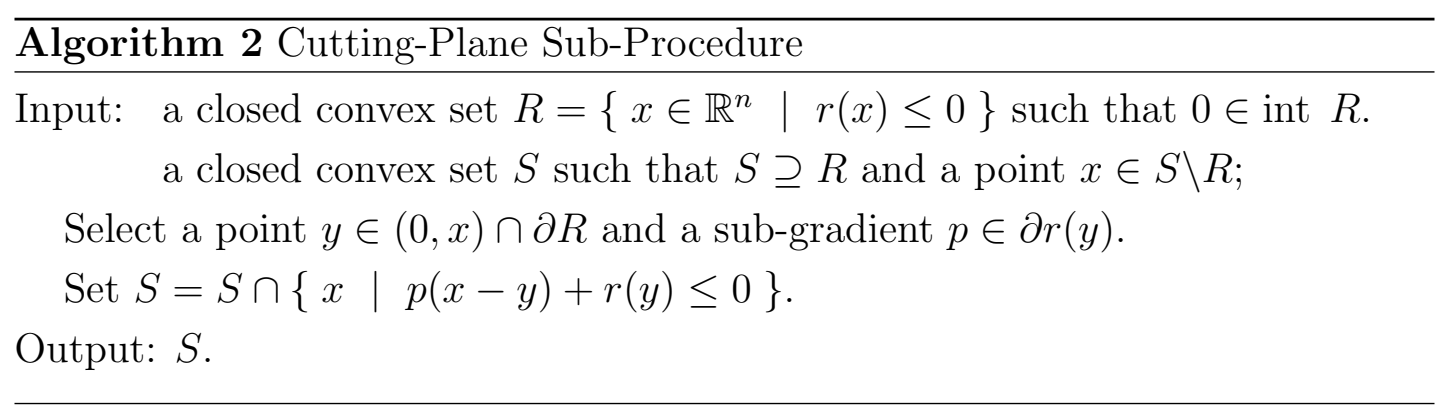

It is worth remarking that condition $0 \in \operatorname{int} R$ is required to ensure that $y \neq x$, and therefore that the hyperplane actually separates $R$ and $x$ strictly; a simple counterexample like $R=\left\{(u, v) \mid(u+1)^{2}+v^{2} \leq 1\right\} \subseteq \mathbb{R}^{2}, S=[-1,1] \times[-1,1]$ and $x=(1,0)$ shows that, failing the hypothesis, the only possible separating hyperplane $u=0$ fails to remove $x$ from $S$. In our setting, the condition is satisfied for $D(\gamma)$ : this is due to (1.18) and to the fact that $\gamma \geq \gamma^{*}>0$, itself 
a consequence of the boundedness of $C$ as discussed before. Boundedness of $C$ is also equivalent to $0 \in \operatorname{int} C^{*}$; therefore, the condition is a fortiori true for $S$ and $Q$, the sets Sub-procedure 2 will be called upon, due to (2.3) and (2.2), respectively.

\subsubsection{A Generic Outer Approximation Sub-Procedure}

We can now define a generic outer approximation procedure which, only provided with an approximate oracle $\Theta$, allows implementations of Algorithm 1 which attain the sufficient convergence conditions introduced in Paragraph 2.3.1. We call this a "generic" outer approximation procedure because it depends on two parameters: a selection rule $\Psi$ for the $x$ and $w$ variables, and a stopping criterion $\Upsilon$. In this paragraph we will describe the properties of the sub-procedure which are independent of the choices of $\Psi$ and $\Upsilon$; later on we will show several different possible choices for these, leading to different implementable algorithms.

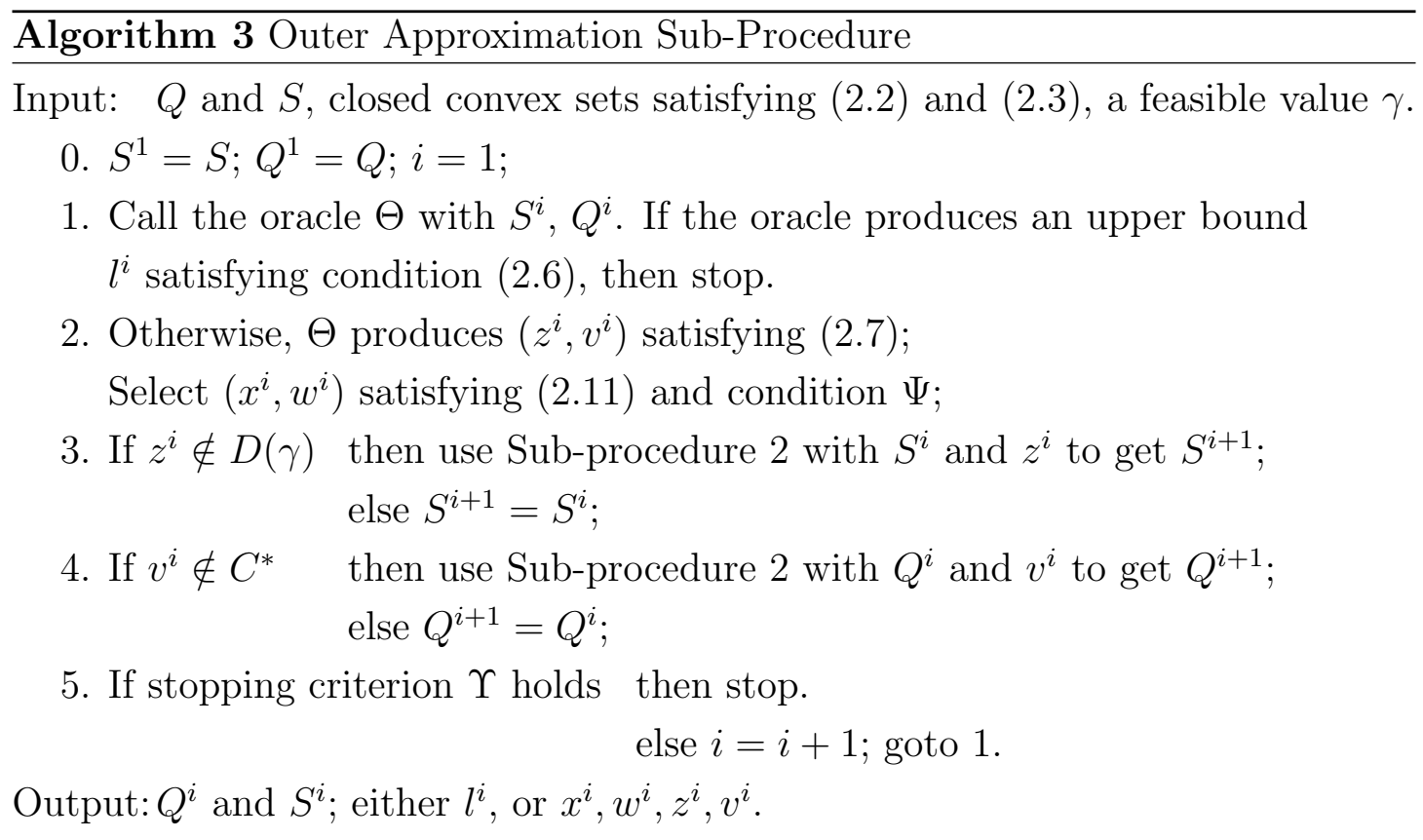

Some remarks on Sub-procedure 3 are in order:

- In Step 4 , since $w^{i} \in \partial C^{*}$ the set $Q^{i}$ can be updated as

$$
Q^{i+1}=Q^{i} \cap\left\{v \mid v x^{i} \leq 1\right\}
$$


In fact, let $\tilde{g}$ be the gauge function of $C^{*}$; it is well-known [80, Theorem 14.5] that $\tilde{g}$ is also the support function of $C^{* *}=C$, that is

$$
C^{*}=\left\{v \in \mathbb{R}^{n} \mid \tilde{g}(v)-1 \leq 0\right\}
$$

and $\tilde{g}(v)=\max \{v x \quad \mid x \in C\}$. Since $x^{i} \in \partial C$ and $w^{i} x^{i}=1$, we have $\tilde{g}\left(w^{i}\right)=w^{i} x^{i}$; therefore, $x^{i} \in \partial\left(\tilde{g}\left(w^{i}\right)-1\right)$ and (2.20) turns out to be a special case of Sub-procedure 2.

- Conditions (2.3) and (2.2) imply that $D(\gamma)$ and $C^{*}$ are included in $S^{i}$ and $Q^{i}$, respectively, for $i=1$. The cutting-plane Sub-procedure 2 ensures this is still true for any $i$, and we get "non-increasing" sequences $\left\{S^{i}\right\}$ and $\left\{Q^{i}\right\}$

$$
\begin{gathered}
D(\gamma) \subseteq \cdots \subseteq S^{i+1} \subseteq S^{i} \subseteq \cdots \subseteq S^{1} \\
C^{*} \subseteq \cdots \subseteq Q^{i+1} \subseteq Q^{i} \subseteq \cdots \subseteq Q^{1}
\end{gathered}
$$

We can now prove the basic properties of Sub-procedure 3 which are independent of the choice of $\Psi$ and $\Upsilon$.

Lemma 2.3.2 If Sub-procedure 3 never ends, then all the cluster points of $\left\{z^{i}\right\}$ and $\left\{v^{i}\right\}$ belongs to $D(\gamma)$ and $C^{*}$, respectively.

Proof. Sub-procedure 3 generates two sequences of points $\left\{z^{i}\right\}$ and $\left\{v^{i}\right\}$ such that $z^{i} \in S^{i}, v^{i} \in Q^{i}$, and the assumptions of Theorem 2.3.1 are satisfied; hence, all the cluster points of $\left\{z^{i}\right\}$ and $\left\{v^{i}\right\}$ belong to $D(\gamma)$ and $C^{*}$, respectively.

It will be crucial to ensure that the sequences $\left\{z^{i}\right\}$ and $\left\{v^{i}\right\}$ do indeed have cluster points. As both $D(\gamma)$ and $C^{*}$ are assumed to be compact, the former a consequence of the boundedness of $C$, the latter coming directly from (1.18). It is natural to suppose also that

$$
\left\{z^{i}\right\} \text { and }\left\{v^{i}\right\} \text { are bounded }
$$

In fact, this condition holds, for instance, if $S^{1}$ and $Q^{1}$ are compact, which is not a restrictive assumption as $D(\gamma)$ and $C^{*}$ are compact too. Therefore, from now onwards we suppose that (2.23) always holds. Note that the sequences $\left\{x^{k}\right\}$ and $\left\{w^{k}\right\}$ are always bounded as due to (2.11) they belong to bounded sets $C$ and $C^{*}$, respectively. 
Corollary 2.3.1 If $\varepsilon^{\prime}>0$, and Sub-procedure 3 never ends, then no cluster point of $\left\{z^{i}\right\}$ belongs to $C$.

Proof. By Lemma 2.3.2, all cluster points of $\left\{v^{i}\right\}$ and (2.23) guarantees that at least one exists. If there were a cluster point of $\left\{z^{i}\right\}$ in $C$, one would have that $\liminf _{i \rightarrow \infty} v^{i} z^{i} \leq 1$, in contradiction with $v^{i} z^{i}-1>\varepsilon^{\prime}$, which is guaranteed by the oracle for any i(cf. (2.7)).

Proposition 2.3.2 If $\varepsilon^{\prime}>0$ and $D(\gamma) \subseteq C$, then Sub-procedure 3 stops after a finite number of iterations.

Proof. Suppose Sub-procedure 3 never ends; due to (2.23), the sequence $\left\{\left(z^{i}, v^{i}\right)\right\}$ has at least a cluster point which belongs to $D(\gamma) \times C^{*}$ by Lemma 2.3.2. Since $D(\gamma) \subseteq C$, then all the cluster points actually belong to $C \times C^{*}$; therefore, we have $\liminf _{i \rightarrow \infty} v^{i} z^{i} \leq 1$ which yields a contradiction as in Corollary 2.3.1.

Remark 2.3.1 The above proofs show the need of requiring $\varepsilon^{\prime}>0$, since for $\varepsilon^{\prime}=0$ the Sub-procedure may never stop. In other words, Sub-procedure 3 can not finitely prove that the optimal value is optimal; this is why it is relevant to clarify the relationship between approximated optimal values and the optimal value.

Finally, it is useful to remark that while condition (2.17) is characteristic of optimizing sequences, it holds for every fixed $\gamma$ by substituting $x^{i}$ to $z^{i}$, even if $\varepsilon^{\prime}=0$.

Lemma 2.3.3 If Sub-procedure 3 never ends, then $\lim \sup _{i \rightarrow \infty} v^{i} x^{i} \leq 1$.

Proof. Lemma 2.3.2 guarantees that all the cluster points of $\left\{v^{i}\right\}$ belong to $C^{*}$. Since $x^{i} \in C$ for all $i$, the thesis follows immediately.

The sub-procedure can then be used to define implementable versions of the Prototype Algorithm 1.

Some remarks on Algorithm 4 are in order: 


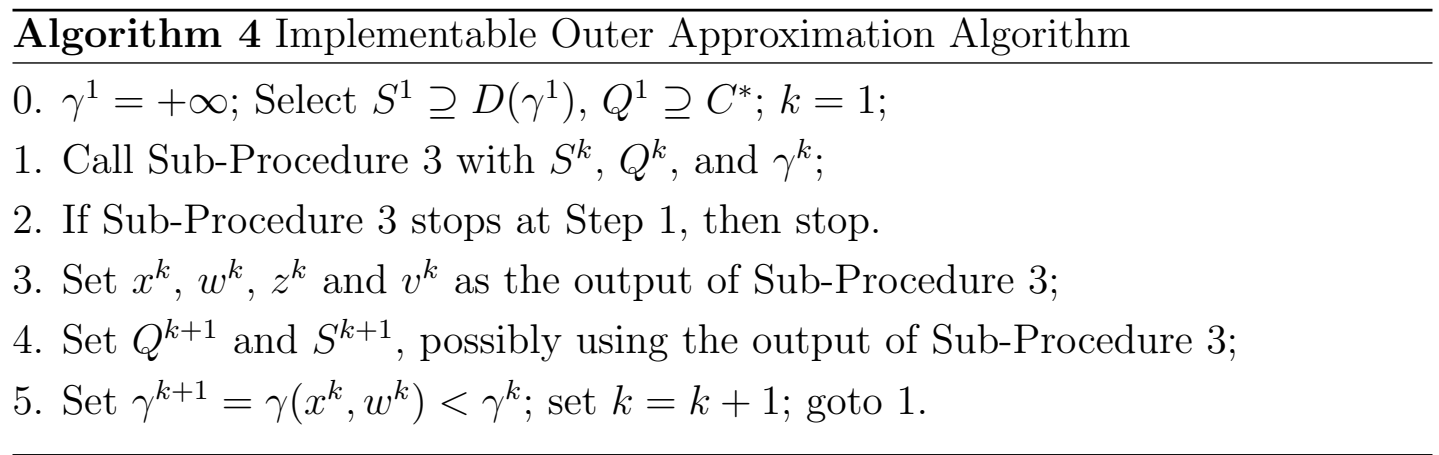

- clearly, due to (2.7), (2.21) and (2.22), condition (2.16) is always satisfied by all possible variants of the algorithm, i.e. irrespective of the concrete choices for $\Psi$ and $\Upsilon$;

- at Step 4, the obvious possibility for $Q^{k+1}$ and $S^{k+1}$ is to set them as the $Q^{i}$ and $S^{i}$ produced by Sub-Procedure 3; since this leads to accumulation in $Q^{k}$ and $S^{k}$ of all cutting planes generated along the iterates, and therefore possibly to "large" descriptions of $Q^{k}$ and $S^{k}$;

- which implementation of $\gamma\left(x^{k}, w^{k}\right)$ has to be chosen depends on the properties of the points $x^{k}$ and $w^{k}$ (see Table 2.1), and therefore ultimately on $\Psi$.

The following sections are devoted to the study of which conditions $\Psi$ and $\Upsilon$ result in a globally convergent Algorithm 4.

\subsubsection{Algorithms Exploiting the Set of Conditions $B_{1}$}

While the oracle in Subprocedure 3 guarantees (2.16), condition (2.18) has to be achieved through additional properties. The algorithms of this subsection will require $(2.18 \mathrm{~b})$ more or less directly and will obtain $(2.18 \mathrm{a})$ by imposing $(2.19 \mathrm{~b})$ and one extra condition, which simply requires $x^{k}$ and $z^{k}$ to be collinear:

$$
z^{k}=\mu_{1}^{k} x^{k} \quad \text { for some } \mu_{1}^{k}>0 .
$$

Lemma 2.3.4 If (2.24) holds for all $k$, then (2.19b) implies (2.18a).

Proof. Due to $(2.24)$ and $w^{k} x^{k}=1,(2.19 \mathrm{~b})$ reads $\limsup _{k \rightarrow \infty} \mu_{1}^{k} \leq 1$, thus we have

$$
\limsup _{k \rightarrow \infty} v^{k}\left(z^{k}-x^{k}\right)=\limsup _{k \rightarrow \infty}\left(\mu_{1}^{k}-1\right) v^{k} x^{k} \leq 0
$$


where the inequality is due to boundedness of the sequences $\left\{v^{k}\right\}$ and $\left\{x^{k}\right\}$.

All algorithms in this paragraph will exploit condition (2.24). Together with (2.11), this forces to choose $x^{k} \in\left\{\alpha z^{k} \mid \alpha \geq 0\right\} \cap \partial C$, thereby basically making the choice of $x^{k}$ automatic once $z^{k}$ is known. Note that the intersection is nonempty due to boundedness of $C$, and therefore $x^{k}$ is always well defined.

The easiest way to guarantee that the sequences generated by Algorithm 4 satisfy (2.24) is to impose that $z^{i}$ and $x^{i}$ are always collinear in Subprocedure 3. Furthermore, this allows to prove that Subprocedure 3 either attains a decrease of the objective function or detects approximate optimality in a finite number of steps, provided that $d z^{i} \leq \gamma$.

Lemma 2.3.5 Suppose $S^{1} \subseteq\left\{z \in \mathbb{R}^{n} \mid d z \leq \gamma\right\}$ and set

$$
\Psi \equiv\left[z^{i}=\mu_{1}^{i} x^{i} \quad \text { with } \mu_{1}^{i}>0\right]
$$

If $\varepsilon^{\prime}>0$ and Subprocedure 3 never ends, then it produces iterates satisfying $x^{i} \in\left(0, z^{i}\right) \cap \Omega, z^{i} \notin C$ and $\gamma\left(x^{i}, w^{i}\right)<\gamma$ for sufficiently large $i$.

Proof. Lemma 2.3.2 guarantees that all the cluster points of $\left\{z^{i}\right\}$ and $\left\{v^{i}\right\}$ belong to $D(\gamma)$ and $C^{*}$, and Corollary 2.3.1 guarantees that each cluster point $\bar{z}$ of $\left\{z^{i}\right\}$ does not belong to $C$, therefore $\bar{z} \in \Omega \backslash C$. Thus, there exists $\bar{x} \in(0, \bar{z})$ such that $\bar{x}$ is a cluster point of $\left\{x^{i}\right\}$. By eventually taking the appropriate subsequences, suppose $z^{i} \rightarrow \bar{z}$ and $x^{i} \rightarrow \bar{x}$. All the above implies that $x^{i} \in\left(0, z^{i}\right)$ and $z^{i} \notin C$ for all sufficiently large $i$. Since $0 \in \operatorname{int} \Omega$ and $\bar{z} \in \Omega$, we have also $\bar{x} \in \operatorname{int} \Omega$ and therefore, $x^{i} \in \Omega$ for all sufficiently large $i$. Hence, we have $\gamma\left(x^{i}, w^{i}\right) \leq d x^{i}<d z^{i} \leq \gamma$ as $z^{i} \in S^{i} \subseteq S^{1}$.

The assumption on $S^{1}$ is actually a mild assumption on how $S^{k}$ is updated in Algorithm 4: it is enough to keep the "objective cut" $d z \leq \gamma^{k}$ among the inequalities which define $S^{k}$ and update it at each iteration to the current value of $\gamma^{k}$. Furthermore, this assumption implies that the membership test in Step 3 of Subprocedure 3 can be reduced to $z^{i} \notin \Omega$.

Some of the properties guaranteed by the above Lemma can be exploited in the stopping criterion $\Upsilon$. Anyway, in order to guarantee that the decrease guaranteed by Subprocedure 3 under (2.24) is "sufficient", one has to prove also 
that the set of conditions $B_{1}$ is satisfied: this requires (2.18), which will be achieved through $(2.18 \mathrm{~b})$ and $(2.19 \mathrm{~b})$. In the next subsections we develop four different ways in which this can be done.

\section{Algorithm $C_{1}$}

The first possibility, directly inspired by the algorithms already proposed in the literature (see, for instance, [113]), is to resort to the following conditions:

$$
\begin{gathered}
d z^{k} \leq \gamma^{k}, \\
x^{k} \in\left(0, z^{k}\right) \cap \Omega \cap \partial C .
\end{gathered}
$$

Condition (2.26) implies (2.24) with $\mu_{1}^{k}>1$. Actually, the two conditions are equivalent if $z^{k} \notin C$ and $x^{k} \in \Omega$ (since we always have $x^{k} \in \partial C$ ), anyway we don't ask for these two conditions. As (2.26) guarantees that the sequence of points $\left\{x^{k}\right\}$ is feasible, we can set $\gamma\left(x^{k}, w^{k}\right)=d x^{k}$.

Lemma 2.3.6 If $\gamma^{*}>0$ and (2.25), (2.26) hold, then (2.19b) holds.

Proof. Since $x^{h}$ is feasible, we have

$$
d x^{0}-\sum_{k=1}^{h}\left(d x^{k-1}-d x^{k}\right)=d x^{h} \geq \gamma^{*}
$$

and therefore

$$
d x^{0}-\gamma^{*} \geq \sum_{k=1}^{h}\left(d x^{k-1}-d x^{k}\right) \geq \sum_{k=1}^{h}\left(d z^{k}-d x^{k}\right)
$$

where the last inequality holds since (2.25) reads $d z^{k} \leq \gamma^{k}=d x^{k-1}$. Taking the limit, we get

$$
\lim _{h \rightarrow+\infty} \sum_{k=1}^{h}\left(d z^{k}-d x^{k}\right) \leq d x^{0}-\gamma^{*}<+\infty .
$$

Since $\mu_{1}^{k}>1,(2.24)$ implies $d z^{k}-d x^{k}>0$ and therefore we get $d z^{k}-d x^{k}=$ $\left(\mu_{1}^{k}-1\right) d x^{k} \rightarrow 0$, which implies that $\lim _{k \rightarrow \infty} \mu_{1}^{k}=1$ since the feasibility of $x^{k}$ gives $d x^{k} \geq \gamma^{*}>0$. Therefore, we have

$$
\limsup _{k \rightarrow \infty} w^{k} z^{k}=\limsup _{k \rightarrow \infty} \mu_{1}^{k} w^{k} x^{k}=\limsup _{k \rightarrow \infty} \mu_{1}^{k} \leq 1
$$


since (2.11) guarantees $w^{k} x^{k}=1$.

Therefore, we can define the following set of conditions

$$
C_{1} \equiv(2.16) \wedge(2.18 b) \wedge(2.25) \wedge(2.26)
$$

which implies $B_{1}$ and thus guarantees convergence for Algorithm 4. The proper choice of $\Psi$ and $\Upsilon$ ensures that these conditions are finitely attained within Subprocedure 3 except $(2.18 \mathrm{~b})$, which requires the knowledge of the entire sequences generated by Algorithm 4. Therefore, we consider a positive sequence $\sigma^{k} \rightarrow 0$ and ask for the subprocedure to provide points $v^{i}$ and $x^{i}$ such that

$$
v^{i} x^{i} \leq 1+\sigma^{k}
$$

This condition can be considered an appropriate formulation of (2.18b) within Subprocedure 3 as in this way Algorithm 4 will surely satisfy (2.18b).

Proposition 2.3.3 Suppose $S^{1} \subseteq\left\{z \in \mathbb{R}^{n} \mid d z \leq \gamma\right\}$ and set

$$
\Psi \equiv\left[z^{i}=\mu_{1}^{i} x^{i} \quad \text { with } \mu_{1}^{i}>0\right], \quad \Upsilon \equiv\left[x^{i} \in \Omega\right] \wedge\left[v^{i} x^{i} \leq 1+\sigma^{k}\right] .
$$

If $\varepsilon^{\prime}>\sigma^{k}>0$, then Subprocedure 3 ends in a finite number of steps; if it stops at Step 5, it reports points $x^{i}, w^{i}, z^{i}$ and $v^{i}$ satisfying the set of conditions $C_{1}$.

Proof. Lemma 2.3.5 and Lemma 2.3.3 guarantee that the stopping criterion $\Upsilon$ will be satisfied for $i$ large enough, independently from the choice of $\sigma^{k}$. Therefore, Subprocedure 3 ends in a finite number of steps. Suppose it ends at Step 5. The stopping criterion $\Upsilon$ directly guarantees (2.18b); (2.16) holds as all iterates satisfy (2.7); (2.25) follows immediately from the assumption on $S^{1}$ as $S^{i} \subseteq S^{1}$. Finally, the stopping criterion $\Upsilon$ and (2.7) allow to get

$$
0<v^{i} x^{i} \leq 1+\sigma^{k}<1+\varepsilon^{\prime} \leq v^{i} z^{i}=\mu_{1}^{i} v^{i} x^{i}
$$

which implies $\mu_{1}^{i}>1$ and thus we have $z^{i} \notin C$. Therefore, $x^{i} \in\left(0, z^{i}\right) \cap \partial C$ and hence (2.26) holds since the stopping criterion $\Upsilon$ provides $x^{i} \in \Omega$.

For this algorithm to work, the sequence $\left\{\sigma^{k}\right\}$ has to be defined explicitly, either a-priori or dynamically as it is used to stop Subprocedure 3. Unlike most algorithms in the literature, it is not needed to require $\mu_{1}^{i}>1$ at every iteration within the subprocedure, thus leaving a wider freedom of choice. 


\section{Algorithm $C_{2}$}

An alternative way to obtain $(2.18 \mathrm{~b})$ is to require

$$
v^{k} x^{h} \leq 1 \quad \text { for all } h<k .
$$

Lemma 2.3.7 If (2.27) holds, then (2.18b) holds.

Proof. Ab absurdo, suppose $v^{k} x^{k}>1+\delta$ for infinitely many $k$ and a given $\delta>0$. Since $\left\{v^{k}\right\}$ and $\left\{x^{k}\right\}$ are bounded, we can suppose $v^{k} \rightarrow \bar{v}$ and $x^{k} \rightarrow \bar{x}$ (eventually taking the appropriate subsequences). Condition (2.11) implies that $\bar{v} x^{h} \leq 1$ for all $h$ and therefore $\bar{v} \bar{x} \leq 1$, a contradiction.

Therefore, we can define the set of conditions

$$
C_{2} \equiv(2.16) \wedge(2.25) \wedge(2.26) \wedge(2.27)
$$

which implies $C_{1}$ and therefore $B_{1}$, thus ensuring convergence for Algorithm 4 .

Clearly, condition (2.27) is guaranteed if

$$
Q^{k} \subseteq \bigcap_{h<k}\left\{v \in \mathbb{R}^{n} \mid v x^{h} \leq 1\right\} .
$$

This can be easily achieved updating $Q^{k+1}$ in Step 4 of Algorithm 4 as follows:

$$
Q^{k+1}=Q^{i} \cap\left\{v \in \mathbb{R}^{n} \mid v x^{i} \leq 1\right\},
$$

where $Q^{i}$ and $x^{i}$ are those produced at the end Subprocedure 3 .

Lemma 2.3.8 If (2.29) holds, then $C^{*} \subseteq Q^{k+1}$.

Proof. Subprocedure 3 guarantees $C^{*} \subseteq Q^{i}$. If we consider the support function of $C$, namely

$$
\sigma_{C}(v):=\max \{v x \mid x \in C\},
$$

then we have

$$
C^{*}=\left\{v \in \mathbb{R}^{n} \mid \sigma_{C}(v)-1 \leq 0\right\} .
$$

Since (2.11) guarantees $x^{i} \in C$, any $v \in C^{*}$ satisfies $v x^{i} \leq \sigma_{C}(v) \leq 1$. 
In this way all the inequalities produced by the Subprocedure 3 are kept: the "quality" of $Q^{k+1}$ may improve, reducing the number of iterations required to stop the subprocedure, but it is likely to increase the cost of each iteration; the practical impact of this trade-off could be gauged only experimentally. In any case, in (2.29) it is always possible to replace $Q^{i}$ with $Q^{k}$ or any intermediate $Q^{j}$ produced by the subprocedure since they both contain $C^{*}$.

Again, an implementable version of the Algorithm 4 can be obtained by choosing $\Psi$ and $\Upsilon$ properly.

\section{Proposition 2.3.4 Set}

$$
\Psi \equiv\left[z^{i}=\mu_{1}^{i} x^{i} \quad \text { with } \mu_{1}^{i}>0\right], \quad \Upsilon \equiv\left[x^{i} \in \Omega\right] \wedge\left[z^{i} \notin C\right]
$$

If $\varepsilon^{\prime}>0$ and (2.28) holds, then Subprocedure 3 ends in a finite number of steps; if it stops at Step 5, it reports points $x^{i}, w^{i}, z^{i}$ and $v^{i}$ satisfying the set of conditions $\mathrm{C}_{2}$.

Proof. Analogous to that of Proposition 2.3.3, considering that (2.27) follows from (2.28) and that $x^{i} \in \Omega$ and $z^{i} \notin C$ imply (2.26).

\section{Algorithm $C_{3}$}

Lemma 2.3.7 states that condition (2.18b) is implied by condition (2.27) under our boundedness assumptions. Symmetrically, we can prove the following result in the same way.

Lemma 2.3.9 If

$$
z^{k} w^{h} \leq 1 \quad \text { for all } h<k
$$

hold for all $k$, then (2.19b) holds.

Proof. Ab absurdo suppose that $w^{k} z^{k} \geq 1+\varepsilon$ for infinitely many $k$ and a given $\varepsilon>0$. Since $\left\{w^{k}\right\}$ and $\left\{z^{k}\right\}$ are bounded, by taking suitable subsequences we have $w^{k} \rightarrow \bar{w}$ and $z^{k} \rightarrow \bar{z}$. Since $w^{h} z^{k} \leq 1$ for all $h<k$, we have $w^{h} \bar{z} \leq 1$ for all $h$, and thus $\bar{w} \bar{z} \leq 1$, a contradiction. 
Therefore, we can define the set of conditions

$$
C_{3} \equiv(2.16) \wedge(2.18 b) \wedge(2.24) \wedge(2.30)
$$

which implies $B_{1}$ (and thus guarantees convergence for Algorithm 4) as (2.24) and (2.30) imply (2.18a) by combining Lemmas 2.3.9 and 2.3.4.

Clearly, (2.30) is guaranteed if

$$
S^{k} \subseteq \bigcap_{h<k}\left\{z \in \mathbb{R}^{n} \mid w^{h} z \leq 1\right\} .
$$

This is easily obtained, for instance, by implementing Step 4 of Algorithm 4 as

$$
S^{k+1}=S^{i} \cap\left\{z \in \mathbb{R}^{n} \mid w^{i} z \leq 1\right\}
$$

where $S^{i}$ and $w^{i}$ are those produced at the end Subprocedure 3, but it is always possible to replace $S^{i}$ with $S^{k}$ or any intermediate $S^{j}$ produced by the subprocedure. Anyway, the current value has to be updated through $\zeta$ in order to guarantee that $S^{k+1}$ outer approximates $D\left(\gamma^{k+1}\right)$.

Lemma 2.3.10 Suppose $\gamma(x, w)=\zeta(w)$. If (2.32) is used in Algorithm 4, then $D\left(\gamma^{k}\right) \subseteq S^{k}$ for all $k$.

Proof. The proof is by induction on the iterate index $k$. If $k=1$, the thesis is guaranteed by the choice of the input data. Suppose the thesis holds for a given $k$ and there exists $\bar{x} \in D\left(\gamma^{k+1}\right)$ such that $\bar{x} \notin S^{k+1}$ : we have

$$
\bar{x} \in D\left(\gamma^{k+1}\right) \subseteq D\left(\gamma^{k}\right) \subseteq S^{i}
$$

where the last inclusion is guaranteed by the way Subprocedure 3 updates $S^{k}$. Therefore, (2.32) implies $w^{i} \bar{x}>1$. Since $\bar{x} \in \Omega$, then $\hat{x}:=\left(w^{i} \bar{x}\right)^{-1} \bar{x} \in \Omega$ (as $w^{i} \bar{x}>1$ and $0 \in \Omega$ ). Moreover, $w^{i} \hat{x}=1$ and therefore $\gamma^{k+1} \leq d \hat{x}<d \bar{x}$ providing the contradiction $\bar{x} \notin D\left(\gamma^{k+1}\right)$.

Again, an implementable version of Algorithm 4 can be obtained by choosing $\Psi$ and $\Upsilon$ properly. Note that the correctness of this version requires $\gamma(x, w)=$ $\zeta(w)$; besides, there is no guarantee that $x^{k}$ is feasible. 
Proposition 2.3.5 Set

$$
\Psi \equiv\left[z^{i}=\mu_{1}^{i} x^{i} \quad \text { with } \quad \mu_{1}^{i}>0\right], \quad \Upsilon \equiv\left[\zeta\left(w^{i}\right)<\gamma\right] \wedge\left[v^{i} x^{i} \leq 1+\sigma^{k}\right]
$$

If $\varepsilon^{\prime}, \sigma^{k}>0$ and (2.31) holds, then Subprocedure 3 ends in a finite number of steps; if it stops at Step 5, it reports points $x^{i}, w^{i}, z^{i}$ and $v^{i}$ satisfying the set of conditions $C_{3}$.

Proof. Analogous to that of Proposition 2.3.3, considering that (2.24) comes by $\Psi$ and that (2.30) is implied by (2.31).

Like Algorithm $C_{1}$, one has to use a sequence $\sigma^{k}$ converging to zero explicitly; in this case, however, it is not required $\sigma^{k}<\varepsilon^{\prime}$, at least initially.

\section{Algorithm $C_{4}$}

The sets of conditions $C_{2}$ and $C_{3}$ are two independent modifications of $C_{1}$; the specific update (2.29) for $Q^{k+1}$ is exploited for the former, while the "symmetric" update (2.32) for $S^{k+1}$ is exploited for the latter. The two modifications can be combined: the set of conditions

$$
C_{4} \equiv(2.16) \wedge(2.27) \wedge(2.24) \wedge(2.30)
$$

implies $B_{1}$ thanks to Lemmas 2.3.7, 2.3.9 and 2.3.4, thus ensuring convergence for Algorithm 4. The following result provides an implementable version of the algorithm.

\section{Proposition 2.3.6 Set}

$$
\Psi \equiv\left[z^{i}=\mu_{1}^{i} x^{i} \quad \text { with } \mu_{1}^{i}>0\right], \quad \Upsilon \equiv\left[\zeta\left(w^{i}\right)<\gamma\right]
$$

If $\varepsilon^{\prime}>0$, (2.28) and (2.31) hold, then Subprocedure 3 ends in a finite number of steps; if it stops at Step 5, it reports points $x^{i}, w^{i}, z^{i}$ and $v^{i}$ satisfying the set of conditions $C_{4}$. 


\subsubsection{Algorithms Exploiting the Set of Conditions $B_{2}$}

The algorithms of this subsection need (2.19) instead of (2.18). As (2.19b) has been exploited to achieve (2.18a), simmetrically (2.19a) can be obtained through (2.18b), relying on the "polar counterpart" of (2.24), namely

$$
v^{k}=\mu_{2}^{k} w^{k} \quad \text { for some } \mu_{2}^{k}>0 \text {. }
$$

Together with (2.11), this forces to choose $w^{k} \in\left\{\alpha v^{k} \mid \alpha \geq 0\right\} \cap \partial C^{*}$, thereby basically fixing $w^{k}$ once $v^{k}$ is known. Note that this intersection is always nonempty since $C^{*}$ is compact.

Lemma 2.3.11 If (2.33) holds for all $k$, then (2.18b) implies (2.19a).

Proof. Due to $(2.33)$ and $w^{k} x^{k}=1,(2.18 \mathrm{~b})$ reads $\limsup _{k \rightarrow \infty} \mu_{1}^{k} \leq 1$, thus we have

$$
\limsup _{k \rightarrow \infty}\left(v^{k}-w^{k}\right) z^{k}=\limsup _{k \rightarrow \infty}\left(\mu_{2}^{k}-1\right) w^{k} z^{k} \leq 0
$$

where the inequality is due to the boundedness of $\left\{z^{k}\right\}$ and $\left\{w^{k}\right\}$.

The algorithms of this subsection will exploit (2.33). The easiest way to guarantee that the sequences generated by Algorithm 4 satisfy it is to impose that $w^{i}$ and $v^{i}$ are always collinear in Subprocedure 3.

Lemma 2.3.12 Suppose $S^{1} \subseteq\left\{z \in \mathbb{R}^{n} \mid d z \leq \gamma\right\}$ and set

$$
\Psi \equiv\left[v^{i}=\mu_{2}^{i} w^{i} \quad \text { with } \quad \mu_{2}^{i}>0\right]
$$

If $\varepsilon^{\prime}>0$ and Subprocedure 3 never ends, then it produces iterates satisfying $\zeta\left(w^{i}\right)<\gamma$ for sufficiently large $i$.

Proof. Taking the appropriate subsequences, we can suppose $w^{i} \rightarrow \bar{w}, v^{i} \rightarrow \bar{v}$ and $z^{i} \rightarrow \bar{z}$. The collineary assumption $\Psi$ implies that $\bar{v}=\bar{\mu} \bar{w}$ for some $\bar{\mu} \geq 0$ and condition (2.7) guarantees $\bar{\mu} \neq 0$. Lemma 2.3 .2 guarantees $\bar{v} \in C^{*}$; since $w^{i} \in \partial C^{*}$, we have $\bar{w} \in \partial C^{*}$ and thus $\bar{\mu} \in(0,1]$. Therefore, we have

$$
\lim _{i \rightarrow \infty} w^{i} z^{i}=\bar{w} \bar{z}=\bar{\mu}^{-1} \bar{v} \bar{z} \geq \lim _{i \rightarrow \infty} v^{i} z^{i} \geq 1+\varepsilon^{\prime}
$$


where the last inequality is due to (2.7). Therefore, $w^{i} z^{i} \geq 1+\varepsilon^{\prime} / 2$ holds for all sufficiently large $i$. By Theorem 2.3.1 we have $\bar{z} \in \Omega$; since $0 \in$ int $\Omega$, we get $\bar{z}^{i}:=\left(1+\varepsilon^{\prime} / 2\right)^{-1} z^{i} \in \Omega$ for all sufficiently large $i$. Hence, we have $\zeta\left(w^{i}\right) \leq d \bar{z}^{i}<d z^{i} \leq \gamma$ as $w^{i} \bar{z}^{i} \geq 1$ and $z^{i} \in S^{i} \subseteq S^{1}$.

Using the above results, we can develop versions of Algorithm 4, which are "symmetric" to those that rely on the set of conditions $B_{1}$. However, the polar reformulation (1.25) is asymmetric in the sense that only the "original" variables $x$ appear in the objective function. Therefore, only two of those four algorithms can be mirrored in this case. Specifically, we will develop sets of conditions $D_{1}$ and $D_{2}$ corresponding to $C_{3}$ and $C_{4}$, respectively. No algorithms corresponding to $C_{1}$ and $C_{2}$ can be devised since they should exploit the condition

$$
w^{k} \in\left(0, v^{k}\right) \cap C^{*} \cap \partial \Omega^{*},
$$

which is "symmetric" to (2.26). However, it would imply the existence of an optimal solution $\left(x^{*}, w^{*}\right)$ such that $w^{*} \in C^{*} \cap \partial \Omega^{*}$, which is not necessarily true: if you consider (1.25) with $n=1, d=1$ and $\Omega=C^{*}=[-1 / 2,4]$, the unique optimal point is $\left(x^{*}, w^{*}\right)=(1 / 4,4)$ while $C^{*} \cap \partial \Omega^{*}=[-1 / 2,1 / 4]$.

\section{Algorithm $D_{1}$}

We can define the set of conditions

$$
D_{1} \equiv(2.16) \wedge(2.18 b) \wedge(2.30) \wedge(2.33)
$$

in a "symmetric" way with respect to $C_{3}$. Due to Lemmas 2.3.9 and 2.3.11, $D_{1}$ implies $B_{2}$ and therefore it ensures convergence for Algorithm 4. An implementable version can be obtained by choosing $\Psi$ and $\Upsilon$ as follows.

\section{Proposition 2.3.7 Set}

$$
\Psi \equiv\left[v^{i}=\mu_{2}^{i} w^{i} \quad \text { with } \mu_{2}^{i}>0\right], \quad \Upsilon \equiv\left[\zeta\left(w^{i}\right)<\gamma\right] \wedge\left[v^{i} x^{i} \leq 1+\sigma^{k}\right]
$$

If $\varepsilon^{\prime}, \sigma^{k}>0$ and (2.31) holds, then Subprocedure 3 ends in a finite number of steps; if it stops at Step 5, it reports points $x^{i}, w^{i}, z^{i}$ and $v^{i}$ satisfying the set of conditions $D_{1}$. 


\section{Algorithm $D_{2}$}

We can define the set of conditions

$$
D_{2} \equiv(2.16) \wedge(2.27) \wedge(2.30) \wedge(2.33)
$$

in a "symmetric" way with respect to $C_{4}$. Due to Lemmas 2.3.7, 2.3.9 and 2.3.11, $D_{2}$ implies $B_{2}$ and therefore it ensures convergence for Algorithm 4. An implementable version can be obtained by choosing $\Psi$ and $\Upsilon$ as follows.

Proposition 2.3.8 Set

$$
\Psi \equiv\left[v^{i}=\mu_{2}^{i} w^{i} \quad \text { with } \quad \mu_{2}^{i}>0\right], \quad \Upsilon \equiv\left[\zeta\left(w^{i}\right)<\gamma\right]
$$

If $\varepsilon^{\prime}>0$, (2.28) and (2.31) hold, then Subprocedure 3 ends in a finite number of steps; if it stops at Step 5, it reports points $x^{i}, w^{i}, z^{i}$ and $v^{i}$ satisfying the set of conditions $D_{2}$.

\subsubsection{Summary}

We have developed six different implementable versions of Algorithm 4: while they are all based on Subprocedure 3, they differ for the stopping criterion $\Psi$, the condition $\Upsilon$ on the iterates, how the evaluation function $\gamma$ is implemented and the way how $S^{k}$ and $Q^{k}$ are updated. All the considered variants are summerized in Table 2.1.

\begin{tabular}{|c|c|c|c|c|c|}
\hline & $\Psi$ & $\Upsilon$ & $\gamma$ & $Q^{k}$ & $S^{k}$ \\
\hline$C_{1}$ & $z^{i}=\mu_{1}^{i} x^{i}, \quad \mu_{1}^{i}>0$ & $x^{i} \in \Omega \wedge v^{i} x^{i} \leq 1+\sigma^{k}$ & $\theta$ & & \\
\hline$C_{2}$ & $z^{i}=\mu_{1}^{i} x^{i}, \quad \mu_{1}^{i}>0$ & $x^{i} \in \Omega \wedge z^{i} \notin C$ & $\theta$ & $(2.29)$ & \\
\hline$C_{3}$ & $z^{i}=\mu_{1}^{i} x^{i}, \quad \mu_{1}^{i}>0$ & $\zeta\left(w^{i}\right)<\gamma^{k} \wedge v^{i} x^{i} \leq 1+\sigma^{k}$ & $\zeta$ & & $(2.32)$ \\
\hline$C_{4}$ & $z^{i}=\mu_{1}^{i} x^{i}, \quad \mu_{1}^{i}>0$ & $\zeta\left(w^{i}\right)<\gamma^{k}$ & $\zeta$ & $(2.29)$ & $(2.32)$ \\
\hline$D_{1}$ & $v^{i}=\mu_{2}^{i} w^{i}, \quad \mu_{2}^{i}>0$ & $\zeta\left(w^{i}\right)<\gamma^{k} \wedge v^{i} x^{i} \leq 1+\sigma^{k}$ & $\zeta$ & & $(2.32)$ \\
\hline$D_{2}$ & $v^{i}=\mu_{2}^{i} w^{i}, \quad \mu_{2}^{i}>0$ & $\zeta\left(w^{i}\right)<\gamma^{k}$ & $\zeta$ & $(2.29)$ & $(2.32)$ \\
\hline
\end{tabular}

Table 2.1: Summary of implementable versions of Algorithm 4

Now, we want to show that all these algorithms are indeed different, in the sense that they can produce different optimizing sequences even if the same 
instance and the same starting conditions are given. To this aim, we consider problem $(C D C)$ with $n=2, d=(0,1)$ and

$$
\begin{aligned}
& \Omega=\left\{x \in \mathbb{R}^{2} \mid-1 \leq x_{1} \leq 2,-1 \leq x_{2} \leq 5,3 x_{1}-x_{2} \leq 4\right\}, \\
& C=\left\{x \in \mathbb{R}^{2} \mid x_{1}^{2}+x_{2}^{2} \leq 4\right\} .
\end{aligned}
$$

Notice that $\Omega$ is a bounded polyhedron, whose verteces provide the alternative description

$$
\Omega=\operatorname{conv}(\{(1,-1),(-1,-1),(-1,5),(2,5),(2,2)\}) .
$$

It is easy to check that the unique optimal solution is the intersection between the segment $[(1,-1),(2,2)]$ (the boundary of the constraint $3 x_{1}-x_{2} \leq 4$ ) and the boundary of $C$, namely the point $x^{*}=(6+\sqrt{6}, 3 \sqrt{6}-2) / 5 \in \Omega \backslash \operatorname{int} C$. Therefore, the optimal value is $\gamma^{*}=(3 \sqrt{6}-2) / 5 \approx 1.0697$. Note that all standard assumptions are satisfied: (1.18) and (1.19) hold, $C$ is compact while regularity follows from Lemma 1.3.3. Furthermore, the value function $\phi$ is locally Lipschitz at 0 , as $(\delta, 0) \in T\left(\Omega, x^{*}\right)$ and $(\delta, 0) \notin T\left(C, x^{*}\right)$ for any $\delta>0$ (see Theorem 2.2.1).

Considering the polar reformulation (1.25), we have

$$
C^{*}=\left\{w \in \mathbb{R}^{2} \mid 4\left(w_{1}^{2}+w_{2}^{2}\right) \leq 1\right\} .
$$

Since any optimal solution of (1.25) must satisfy $w^{*} x^{*}=1$ and $w^{*} \in \partial C^{*}$, we have that $w^{*}=(6+\sqrt{6}, 3 \sqrt{6}-2) / 20$ provides the only possibility for the polar part of the optimal solution.

In the following, we assume the oracle $\Theta$ to always choose the same $(z, v)$ when $S, Q$ and $\gamma$ are the same; furthermore, we set $\varepsilon=1$ so that the pairs $(z, v)$ satisfying (2.7) must actually be optimal for (2.4). In this way, we eliminate the nondeterminism due to the fact that the oracle may return different $\varepsilon$-optimal solutions of (2.4), which may be "many" especially if $\varepsilon<<1$; nonetheless, the six algorithms all construct different optimizing sequences for this instance.

Consider the following starting situation:

$$
\begin{aligned}
& \sigma^{1}=0.1, \quad \gamma^{1}=+\infty, Q^{1}=[-1 / 2,1 / 2] \times[-1 / 2,1 / 2], \\
S^{1}= & \left\{x \in \mathbb{R}^{2} \mid-1 \leq x_{1} \leq 2,-1 \leq x_{2} \leq 10,3 x_{1}-x_{2} \leq 4\right\} \\
= & \operatorname{conv}(\{(1,-1),(-1,-1),(-1,10),(2,10),(2,2)\}) .
\end{aligned}
$$


All algorithms start call Subprocedure 3 with $S^{1}, Q^{1}$ and $\gamma^{1}$ as input data. The oracle provides an optimal solution of the certificate problem

$$
\max \left\{z v-1 \mid(z, v) \in S^{1} \times Q^{1}\right\}
$$

whcih can be easily obtained comparing the value $\bar{v} \bar{z}$ for all pairs where $\bar{z}$ is an extreme point of $S^{1}$ and $\bar{v}$ is an extreme point of $Q^{1}$. In this case, the unique optimal solution turns out to be $\left(z^{1}, v^{1}\right)=((2,10),(1 / 2,1 / 2))$ with optimal value $v\left(\overline{O C}_{\gamma^{1}}\right)=5$; thus, according to our assumptions, this is the pair the oracle $\Theta$ returns for all algorithms.

Algorithms implementing the set of conditions $B_{1}$. The four algorithms $C_{1}, C_{2}, C_{3}$, and $C_{4}$ ask for $x^{i}$ and $z^{i}$ to be collinear. Due to (2.11) the only possible choice is $x^{1}=(2,10) / \sqrt{26}$; since we have both $z^{1} \notin C$ and $x^{1} \in \Omega$, then the point satisfies also the more restrictive condition (2.26). Due to (2.11) the only choice for the corresponding polar point is $w^{1}=(1,5) / \sqrt{104}$.

The subprocedure stops at this first iteration for algorithms $C_{2}$ and $C_{4}$, since we have $x^{1} \in \Omega, z^{1} \notin C$ and $\zeta\left(w^{1}\right) \leq d x^{1}<\gamma^{1}$. On the contrary, it does not stop for algorithms $C_{1}$ and $C_{3}$ since

$$
v^{1} x^{1}=6 / \sqrt{26} \approx 1.1767>1+\sigma^{1} .
$$

In algorithm $C_{2}$ the subprocedure provides the new current value $\gamma^{2}=\theta\left(x^{1}\right)=$ $d x^{1}=10 / \sqrt{26} \approx 1.9612$ while in algorithm $C_{4}$ it provides $\gamma^{2}$ as

$$
\zeta\left(w^{1}\right)=\min \left\{d x \mid x \in \Omega, x_{1}+5 x_{2} \geq \sqrt{104}\right\}
$$

The optimal solution of the above linear program is $\bar{x}^{1}=(10+\sqrt{26}, 3 \sqrt{26}-2) / 8$ and therefore the current value will be updated to

$$
\gamma^{2}=\zeta\left(w^{1}\right)=d \bar{x}^{1}=(3 \sqrt{26}-2) / 8 \approx 1.6621<10 / \sqrt{26}
$$

As for algorithms $C_{1}$ and $C_{3}$, the subprocedure performs one more iteration after the sets $S^{1}$ and $Q^{1}$ have been updated through subprocedure 2 (since $z^{1} \notin \Omega$ and $\left.v^{1} \notin C\right)$ :

$$
\begin{gathered}
S^{2}=S^{1} \cap\left\{\left(x \in \mathbb{R}^{2} \mid x_{2} \leq 5\right\}=\Omega,\right. \\
Q^{2}=Q^{1} \cap\left\{w \in \mathbb{R}^{2} \mid \sqrt{2}\left(w_{1}+w_{2}\right) \leq 1\right\} .
\end{gathered}
$$


At the second iteration of the subprocedure the oracle returns the (unique) optimal solution of the certificate problem

$$
\max \left\{z v-1 \mid(z, v) \in S^{2} \times Q^{2}\right\}
$$

which is $\left(z^{2}, v^{2}\right)=((2,5),(\sqrt{2}-1,1) / 2)$. Therefore, the collinearity condition $\Psi$ and $(2.11)$ imply $x^{2}=(4,10) / \sqrt{29}$ and $w^{2}=(2,5) / 2 \sqrt{29}$. Since $x^{2} \in \Omega$, $\zeta\left(w^{2}\right) \leq d x^{2}<\gamma^{1}$ and

$$
v^{2} x^{2}=(3+2 \sqrt{2}) / \sqrt{29} \approx 1.0823 \leq 1+\sigma^{1}
$$

the subprocedure stops: algorithm $C_{1}$ selects $\gamma^{2}=\theta\left(x^{2}\right)=d x^{2}=10 / \sqrt{29} \approx$ 1.6569 while algorithm $C_{3}$ solves the linear program

$$
\zeta\left(w^{2}\right)=\min \left\{d x \quad \mid x \in \Omega, 2 x_{1}+5 x_{2} \geq 2 \sqrt{29}\right\}
$$

in order to get the point $\left.\bar{x}^{2}=(20+2 \sqrt{29}, 6 \sqrt{29}-8) / 17\right)$ and set $\gamma^{2}=\zeta\left(w^{2}\right)=$ $d \bar{x}^{2}=(6 \sqrt{29}-8) / 17 \approx 1.4301$.

The four algorithms have all provided different values for $\gamma^{2}$ and therefore they are different from each other.

Algorithms implementing the set of conditions $B_{2}$. The algorithms $D_{1}$ and $D_{2}$ require $w^{i}$ and $v^{i}$ to be collinear. Due to $(2.11)$ the only possible choice is $w^{1}=(1,1) / 2 \sqrt{2}$ and the corresponding point in the original space can be only $x^{1}=(\sqrt{2}, \sqrt{2})$. The subprocedure stops at this first iteration for algorithm $D_{2}$, since we have $x^{1} \in \Omega$ and theerfore $\zeta\left(w^{1}\right) \leq d x^{1}<\gamma^{1}$. On the contrary, it does not stop for algorithm $D_{1}$ since

$$
v^{1} x^{1}=\sqrt{2} \approx 1.4142>1+\sigma^{1} .
$$

In algorithm $D_{2}$ the subprocedure provides the new current value $\gamma^{2}$ as

$$
\zeta\left(w^{1}\right)=\min \left\{d x \quad \mid x \in \Omega, x_{1}+x_{2} \geq 2 \sqrt{2}\right\}=(3-\sqrt{2}) / \sqrt{2} \approx 1.1213 .
$$

and the corresponding optimal solution $\bar{x}^{1}=(1+\sqrt{2}, 3-\sqrt{2}) / \sqrt{2}$ is the best achieved point. Since this value for $\gamma^{2}$ is different from all those seen so far, $D_{2}$ is yet another different algorithm.

In algorithm $D_{1}$ the subprocedure performs a second iteration after the sets $S^{1}$ and $Q^{1}$ have been updated exactly in the same way as in algorithms $C_{1}$ and 
$C_{3}$ (since $z^{1}$ and $v^{1}$ are indeed the same). Therefore, the oracle provides the same $z^{2}=(2,5)$ and $v^{2}=(\sqrt{2}-1,1) / 2$. Due to the collinearity condition $\Psi$ and $(2.11)$, we get $w^{2}=(\sqrt{2}-1,1) / 2 \sqrt{4-2 \sqrt{2}}$ and $x^{2}=\sqrt{2-\sqrt{2}}(1,1+\sqrt{2})$. Since

$$
v^{2} x^{2}=\sqrt{4-2 \sqrt{2}} \approx 1.0824 \leq 1+\sigma^{1}
$$

the subprocedure ends. The value it returns as $\gamma^{2}$ is

$$
\zeta\left(w^{2}\right)=\min \left\{d x \quad \mid x \in \Omega,(\sqrt{2}-1) x_{1}+x_{2} \geq 2 \sqrt{4-2 \sqrt{2}}\right\} \approx 1.4169 .
$$

and the corresponding optimal solution

$$
\bar{x}^{2}=\left(\frac{4+2 \sqrt{4-2 \sqrt{2}}}{2+\sqrt{2}}, \frac{4+6 \sqrt{4-2 \sqrt{2}}-4 \sqrt{2}}{2+\sqrt{2}}\right)
$$

is the best achieved point. Once again, this value for $\gamma^{2}$ is different from all previous ones: all the six algorithms are different.

\subsection{Comparisons and Conclusions}

The algorithms proposed in this thesis are inspired by the seminal works of Tuy $[102,101]$, in which the canonical DC problem has been introduced, it has been shown how any $D C$ problem can be reduced to it, and the first cutting plane algorithm has been proposed. The initial algorithm had less refined convergence properties; by cutting off points such that $d x>\gamma^{k}-\alpha$, for a feasible tolerance $\alpha \geq 0$, the algorithm may terminate with only an $\alpha$-optimal solution. More refined versions of the algorithms, more akin to those presented in this thesis, were presented later. The polyhedral annexation method, proposed in $[110,119]$ for the special case of (CDC) where $\Omega$ is a polyhedron, is the first where the exact form

$$
v^{k} z^{k} \geq \max \left\{v z \mid(z, v) \in D\left(\gamma^{k}\right) \times C^{*}\right\}
$$

of the approximate optimality conditions (2.6) (see also Proposition 2.2.1) has been introduced; afterwards, $[121,113]$ showed that this algorithm can be extended to any (CDC) problem. In [112], the non-slackened "objective cut" (2.25) was introduced, and $\gamma^{1}=+\infty$ was first allowed. A further variant was developed 
in [104] for the "more general" case where $d x$ is replaced by a convex finite-valued function $f(x)$ although this can also be recast as a canonical DC program.

Several attempts at generalizing the results in the above papers were not entirely successful. A variant of [104] has been proposed in [72], where a binary search on the value of $\gamma$ is proposed; this, however, is unnecessary. The algorithm proposed in [95], a modified form of the ones in [102, 101], as well as its modified form in [45], were later shown not to guarantee convergence [112]. Similarly, a counter example disproving convergence was developed in [8] for the cutting plane algorithms of $[6,7]$. Finally, the analogous algorithm of [87], based on a slightly modified form of the classical optimality condition (1.22), was also shown not to be always convergent [98]; besides, the modified optimality condition is not easier to check than (1.22).

All the converging algorithms in the above papers satisfy the set of conditions $C_{1}$ or $C_{2}$, and are special cases of those presented in this thesis. Furthermore, it is basically given for granted that the "oracle" for checking the optimality conditions is realized through enumeration of vertices. The contributions of the present thesis are the following:

- The introduction of "approximate oracle" conditions (2.6)-(2.7), which are designed to allow for more sophisticated and efficient solution procedures, with respect to pure vertex enumeration, to tackle the problem of checking the optimality condition, arguably the computational bottleneck in this type of approaches.

- A thorough study of the impact of approximations in the optimality conditions onto the quality of the approximately optimal solutions satisfying them.

- Full exploitation of the "primal-polar" formulation of the optimality conditions based on (2.1) in order to derive a very general hierarchy of conditions ensuring convergence.

- A general algorithmic scheme based on the developed hierarchy which gives rise to six different implementable algorithms, four of which $\left(C_{3}, C_{4}, D_{1}\right.$ and $D_{2}$ ) do not seem to have previously been considered in the literature; 
each of these algorithms can generate an approximate optimal value in a finite number of steps, where the error can be managed and controlled.

It may be worth remarking that the "new" algorithms $C_{3}, C_{4}, D_{1}$ and $D_{2}$ all use $\gamma(x, w)=\zeta(w)$. This has been inspired by the reformulation of (CDC) as the quasi-concave minimization problem (2.12) already proposed in [123]. However, in that paper a "cut and split" method was used, that is entirely different from the outer approximation algorithms proposed in this thesis. Indeed, that method belongs to the main other family of algorithms for canonical DC problems, that of branch and bound methods (see, for instance, [105, 107, 108]). So, this research has shown how concepts developed for one family of approaches can be useful even for an entirely unrelated one.

While this thesis seems to offer a quite comprehensive convergence theory for "oracle-based" outer approximation algorithms for canonical DC programs, much still needs to be done before these algorithms become widely used and accepted as those based on the branch and bound paradigm. In particular, more work is needed to identify pratically efficient ways to implement the oracle, at least on special types of canonical DC programs in which the sets $\Omega$ and $C$ have some form of exploitable structure; this will be the focus of further research. 


\section{Chapter 3}

\section{Algorithms for Single Reverse Polar Problems}

\subsection{Introduction}

As it has been discussed in Chapter 2, in the last decades, optimization techniques have been widely applied in engineering, economics and other fields. A large number of nonconvex optimization problems can be reduced to DC optimization problems. Furthermore, all DC optimization problems can be transformed to the canonical form (CDC). In turn, (CDC) can be reformulated as (1.25), that is a program whose non-convexity is due to a single constraint which asks for a reverse polar inequality to hold. Under mild assumption, the necessary and sufficient optimality conditions for (CDC) are

$$
\left\{z \in \mathbb{R}^{n} \mid z \in \Omega, d z \leq \gamma\right\} \subseteq \Gamma^{*}
$$

which can be equivalently reformulated as

$$
\max \{v z-1 \mid z \in \Omega, v \in \Gamma, d z \leq \gamma\} \leq 0
$$

providing an "optimization form" of the above optimality conditions. In Chapter 2 , we have developed a family of outer approximation approaches for (1.25) which are based on an approximated oracle for the solution of (3.1). The latter problem has a convex feasible set and a non-concave objective function: by allowing an approximate solution we relax the computational requirements of standard outer 
approximation algorithms, hopefully paving the way for more effective solution approaches to (CDC) in practice.

In this chapter, we propose a novel generalization of the canonical DC optimization problem considering the more general form

$$
(S R P) \quad \min \{d x+e w \mid x \in \Omega, w \in \Gamma, w x \geq 1\}
$$

where $d \in \mathbb{R}^{n}, e \in \mathbb{R}^{n}, \Omega$ and $\Gamma$ are closed convex sets in $\mathbb{R}^{n}$. Unlike the canonical DC case, we don't suppose $0 \in \Omega$ and $0 \in$ int $\Gamma^{*}$. Furthermore, this problem, which we call the Single Reverse Polar problem, differs from (1.25) because of the presence of the term "ew" in the objective function. The rationale behind this definition is that, under proper assumptions, an "optimization form" of the optimality conditions of (3.2) requires the solution of the problem

$$
\max \{v z-1 \mid z \in \Omega, v \in \Gamma, d z+e v \leq \gamma\}
$$

which is a minimal modification of that in (3.1). In particular, the two problems share the same non-concave functions, while the convex feasible set is very similar; only, in (3.3) the single constraint $d z+e v \leq \gamma$ renders the feasible set nonseparable in $z$ and $v$, while in the (1.25) case separability is retained. However, it is likely that this difference does not substantially impact the practical cost of outer approximation approaches. Still, we will show that (SRP) is "substantially different" from (1.25), in the sense that several properties enjoyed be the latter are lost in the former. Since (1.25) is the special case of (SRP) with $e=0$ and $\Gamma=C^{*}$, it is not surprising that the outer approximation approaches for the former need be substantially modified in order to cope with the latter. Our analysis of outer approximation algorithms for (SRP) also sheds some light on the algorithms for the original (CDC).

The chapter is organized as follows. In Section 3.2 we describe analyze the main properties of Problem (SRP) and contrast them with those of its special case (1.25). Then, in Section 3.3 we extend our approximate optimality conditions for (1.25) to the (SRP) case. In Section 3.4, we develop a hierarchy of conditions that guarantee the convergence of cutting plane algorithms; relying on these conditions, we build four cutting plane algorithms for solving (SRP), which seem to be new and cannot be reduced to each other. 
Algorithms for Singe Reverse Polar Problems

\subsection{The Single Reverse Polar Problem}

In order to avoid that (SRP) could be reduced a convex minimization problem, we also suppose that the set $\left\{(x, w) \in \mathbb{R}^{n} \times \mathbb{R}^{n} \mid w x \geq 1\right\}$ provides an essential constraint, i.e.

$$
\min \{d x+e w \mid x \in \Omega, w \in \Gamma\}<\min \{d x+e w \mid x \in \Omega, w \in \Gamma, w x \geq 1\}
$$

Relying on an appropriate translation, assumption (3.4) can be equivalently stated through the following two conditions

$$
\begin{gathered}
(\bar{x}, \bar{w}) \in \Omega \times \Gamma \\
d x+e w>d \bar{x}+e \bar{w}=\bar{\gamma} \quad \forall(x, w) \in(\Omega \times \Gamma) \cap\left\{(x, w) \in \mathbb{R}^{n} \times \mathbb{R}^{n} \mid w x \geq 1\right\} .
\end{gathered}
$$

Here $(\bar{x}, \bar{w})$ can be the optimal solution of problem $\min \{d x+e w \mid x \in \Omega, w \in \Gamma\}$. Notice that the interiors of $\Omega$ and $\Gamma$ are not empty, it is easy to deduce that there exists $(\tilde{x}, \tilde{w}) \in \operatorname{int} \Omega \times \operatorname{int} \Gamma$ satisfying (3.6). Therefore, throughout the thesis we assume that $(\bar{x}, \bar{w}) \in$ int $\Omega \times$ int $\Gamma$. As a consequence, any feasible solution $(x, w) \in \Omega \times \Gamma \cap\{(x, w) \mid w x>1\}$ provides a better feasible solution taking the unique intersection between the segment with $(\bar{x}, \bar{w})$ and $(x, w)$ as end points and the boundary of $\{(x, w) \mid w x=1\}$, i.e., $\left(x^{\prime}, w^{\prime}\right) \in(\bar{x}, \bar{w}) \times(x, w) \cap\{(x, w) \mid$ $w x=1\}$ satisfies $d x^{\prime}+e w^{\prime}<d x+e w$. As a consequence, all optimal solutions to (SRP) belongs to $\{(x, w) \mid w x=1\}$.

In order to guarantee the existence of optimal solutions, we assume the boundedness of $\Omega$ and $\Gamma$, which further implies the boundedness of the level sets

$$
R(\gamma):=\{(x, w) \in \Omega \times \Gamma \mid d x+e w \leq \gamma\}
$$

for the feasible value $\gamma$, i.e., those values $\gamma=d x+e w \geq \gamma^{*}$ for some $(x, w) \in$ $\Omega \times \Gamma \cap\{(x, w) \mid w x \geq 1\}$, where

$$
\gamma^{*}:=\min \{d x+e w \mid x \in \Omega, w \in \Gamma, w x \geq 1\}
$$

is the optimal value of the problem. This ensures existence of an optimal solution $\left(x^{*}, w^{*}\right)$ of $(\mathrm{SRP})$, and therefore $\gamma^{*}>\bar{\gamma}$ due to (3.6), a property turn out to be very useful. A simple counterexample like $\Omega=[0,+\infty), \Gamma=[0, \infty), d=1$ and $e=0$; shows that the problem (SRP) may have no optimal solution. 
The level set introduced above is also helpful to check whether a feasible value is optimal or not. In fact, it is straightforward that $\gamma=\gamma^{*}$ implies the following inclusion:

$$
R(\gamma) \subseteq\{(x, w) \mid w x \leq 1\}
$$

The proof of the optimality condition will be given later with a more general form. Furthermore, this necessary optimality condition (3.7) is also sufficient when problem (SRP) is regular, i.e.

$$
\min \{d x+e w \mid(x, w) \in \Omega \times \Gamma, w x \geq 1\}=\inf \{d x+e w \mid(x, w) \in \Omega \times \Gamma, w x>1\}
$$

\subsection{Approximate Optimality Conditions}

Given a feasible value $\gamma$, the optimality condition (3.7) should be checked in order to recognize whether or not $\gamma$ is the optimal value. Unfortunately, there is no known efficient way to check the inclusion between two sets. Yet, any exact algorithm for (SRP) must eventually cope with this problem.

\subsubsection{Optimality Conditions and (Approximate) Oracles}

In order to make (3.7) more readily approachable, we consider the "optimization version" (3.3) of the optimality. Obviously, (3.7) holds if and only if the optimal value $v\left(R O C_{\gamma}\right)$ of $(3.3)$ is less or equal to 0 . Thus the above problem provides a way for checking optimality of a given value $\gamma$. Since the objective function

of (3.3) is not concave, there are no known efficient approaches for this problem as well. However, checking (3.7) through the optimization problem (3.3) has the advantage of making it easy to define a proper notion of approximate optimality conditions.

A first way of approximating problem (3.3) is to replace $\Omega$ and $\Gamma$ by two convex sets $S$ and $Q$, respectively, satisfying

$$
\begin{aligned}
& \Omega \subseteq S . \\
& \Gamma \subseteq Q
\end{aligned}
$$


This is a standard step in cutting plane (outer approximation) approaches, where $S$ and $Q$ are chosen to be "easier" than the original sets (e.g., polyhedra with possibly few vertices or facets) and iteratively refined to become better and better approximations of $\Omega$ and $\Gamma$ as needed. Hence, one considers the relaxation of (3.3)

$$
\left(\overline{R O C}_{\gamma}\right) \quad \max \{v z-1 \mid z \in S, v \in Q, d z+e v \leq \gamma\}
$$

whose optimal value provides an upper bound on $v\left(R O C_{\gamma}\right)$; thus,

$$
v\left(\overline{R O C}_{\gamma}\right) \leq 0
$$

is a convenient sufficient optimality condition for (SRP). If (3.12) does not hold, then either $\gamma$ is not the optimal value, or $S$ and $Q$ are not "good" approximations of $\Omega$ and $\Gamma$, respectively. All the cutting plane algorithms presented in this chapter follow the same basic scheme: (3.11) is solved, and its solution is used to improve $S$ or $Q$ or $\gamma$, in such a way to guarantee convergence of $\gamma$ to the optimal value. The focus of the research is on devising a number of different ways to achieve a convergent algorithm for (SRP) out of an "oracle" for (3.11). However, it is likely that in any such approach the solution of (3.11) is going to be the computational bottleneck; it thus makes sense to consider solving (3.11) only approximately.

Solving (3.11) approximately may actually mean two different things:

1. computing a "large enough" lower bound on $v\left(\overline{R O C}_{\gamma}\right)$, i.e., finding a feasible solution $(\bar{z}, \bar{v})$ "sufficiently close" to the optimal solution;

2. computing a "small enough" upper bound $l \geq v\left(\overline{R O C}_{\gamma}\right)$.

Algorithmically, the two notions correspond to two entirely different classes of approaches: lower bounds are produced by heuristics computing feasible solutions, while upper bounds are produced by solving suitable relaxations of $\left(\overline{R O C}_{\gamma}\right)$, e.g. replacing the non-concave objective function $v z-1$ with a suitable concave upper approximation. Exact algorithms combining the two can then be used to push the lower bound and the upper bound arbitrarily close together. However, for the sake of our approaches only one of the two bounds is needed at any given time. In fact, $v\left(\overline{R O C}_{\gamma}\right)$ is either positive or non-positive. To establish that the first case holds amounts to finding a solution $(\bar{z}, \bar{v})$ to $(3.11)$ such that $\bar{v} \bar{z}-1>0$, while for the second case one needs an upper bound $l \leq 0$. 
This is the rationale behind our definition of an approximate oracle for (3.11). In our development, we will assume availability of a procedure $\Theta$ which, given $S$, $Q, \gamma$, and two positive tolerances $\varepsilon$ and $\varepsilon^{\prime}$

- either produces an upper bound

$$
\varepsilon v\left(\overline{R O C}_{\gamma}\right) \leq l \quad \text { such that } \quad l \leq \varepsilon^{\prime}
$$

- or produces a pair

$$
(\bar{z}, \bar{v}) \text { feasible for }(3.11) \quad \text { such that } \quad \bar{v} \bar{z}-1 \geq \varepsilon v\left(\overline{R O C}_{\gamma}\right)>\varepsilon^{\prime}
$$

Clearly, (3.14) corresponds to a pretty weak requirement about the way in which (3.11) is solved: a solution, which is optimal only with fixed but arbitrary relative tolerance $\varepsilon>0$ and absolute tolerance $\varepsilon^{\prime}$, is required. Condition (3.13) allows the lower bound to be "small enough" but positive, rather than non-negative; this is taken as the stopping condition of the approach, and we will show that the positive tolerance allows for finite termination of the algorithms even when $\gamma$ is optimal. The drawback is that a feasible value $\gamma$ needn't be optimal when (3.13) holds: the next subsection is devoted to the study of the relationships between the "quality" of $\gamma$ and the tolerances $\varepsilon$ and $\varepsilon^{\prime}$.

\subsubsection{Properties of Optimal Solutions}

As problem (CDC) can be viewed as a special case of problem (SRP), thus we may wonder whether the properties of optimal solutions of (CDC) still hold for (SRP) or not. Notice that in problem (CDC), i.e. (SRP) with $0 \in \Omega \cap$ int $\Gamma$ and $e=0$, all optimal solutions $\left(x^{*}, w^{*}\right)$ satisfies $w^{*} x^{*}=1$, or equivalently $\left(x^{*}, w^{*}\right) \in \partial \Gamma^{*} \times \partial \Gamma$, and there exists at least one optimal solution satisfying $x^{*}$ in $\partial \Omega \cap \partial \Gamma^{*}$ when $\partial \Omega \cap \partial \Gamma^{*} \neq \emptyset$.

Theorem 3.3.1 When the dimensions of $\Omega$ and $\Gamma$ are not less than 2, problem $(S R P)$ has at least one optimal solution $\left(x^{*}, w^{*}\right)$ in $\partial \Omega \times \partial \Gamma$.

Proof. Take any optimal solution $\left(x^{1}, w^{1}\right)$, if $\left(x^{1}, w^{1}\right) \in \partial \Omega \times \partial \Gamma$, then the lemma is proved. Otherwise, assume that $x^{1} \in$ int $\Omega$; then, for any $u^{1}$ such that $w^{1} u^{1}=0$ and a sufficiently large number $\lambda$ we have $x^{1} \pm \lambda u^{1} \notin \Omega$. Then there exist 
positive numbers $\lambda_{1}$ and $\lambda_{2}$ such that $x^{2}=x^{1}+\lambda_{1} u^{1}$ and $x^{3}=x^{1}-\lambda_{2} u^{1}$ belong to the boundary of $\Omega$. It is obvious that $\min \left\{d x^{2}, d x^{3}\right\} \leq d x^{1}$ and $w^{1} x^{2}=1$, $w^{1} x^{3}=1$, so either $\left(x^{2}, w^{1}\right)$ or $\left(x^{3}, w^{1}\right)$ is optimal. In the same way, we can find another $w^{2}$ to replace $w^{1}$ if $w^{1} \in$ int $\Gamma$.

When the dimension of $\Omega$ or $\Gamma$ is 1 , then all optimal solution of (SRP) may belong to int $\Omega \times$ int $\Gamma$ : a counter-example is the instance where $\Omega=\Gamma=[0,2]$, $d=e=1$. The optimal solution is $\left(x^{*}, w^{*}\right)=(1,1) \in \operatorname{int} \Omega \times \operatorname{int} \Gamma$.

However, some properties of optimal solutions in (CDC) are lost in problem (SRP). An important observation is, unlike problem (CDC), $x^{*}$ may no longer be in $\partial \Gamma^{*}$ in problem (SRP):

Example 3.3.1 Consider the instance where

$$
\begin{gathered}
\Omega=\left\{\left(x_{1}, x_{2}\right) \mid x_{1}^{2}+\left(x_{2}-1\right)^{2} \leq 4\right\}, \\
\Gamma=\left\{\frac{\left(w_{1}, w_{2}\right)}{(1+\delta)} \mid w_{1}+w_{2} \geq-0.5,2 w_{1}+w_{2} \leq 1,-2 w_{1}+w_{2} \leq 1,-w_{1}+w_{2} \geq-0.5\right\}
\end{gathered}
$$

where $\delta$ is a small enough positive value and $(\bar{x}, \bar{w})=(0,0), d=(0,1), e=(1,0)$.

Take any point $(x, w)$ s.t. $x \in \partial \Gamma^{*}$, we have $x_{2}=1+\delta$ and $w_{1} \geq 0: w x \geq 1$ and $-2 w_{1}+w_{2} \leq 1 / 1+\delta$ implies

$$
1 \leq w_{1} x_{1}+w_{2} x_{2} \leq w_{1} x_{1}+\left(\frac{1}{1+\delta}+2 w_{1}\right)(1+\delta)=1+w_{1}\left(x_{1}+2+2 \delta\right)
$$

which implies that $w_{1}\left(x_{1}+2+2 \delta\right) \geq 0$. As $x_{1}+2+2 \delta \geq 0$, then $w_{1} \geq 0$. Therefore,

$$
d x+e w=x_{2}+w_{1} \geq 1+\delta
$$

However, if we choose $(x, w) \in \Omega \times \Gamma$ s.t. $x=\left(-2 \sqrt{1-\delta^{2}}, 1+2 \delta\right), w=$ $\left(-\delta, 2 \delta+2-2 \sqrt{1-\delta^{2}}\right) /\left[\left(4 \delta+2-2 \sqrt{1-\delta^{2}}\right)(1+\delta)\right]$, We have $w x=1$,

$$
\begin{aligned}
d x & +e w=w_{1}+x_{2}=1+2 \delta-\frac{\delta}{\left[\left(4 \delta+2-2 \sqrt{1-\delta^{2}}\right)(1+\delta)\right.} \\
& =1+\delta+\delta\left(1-\frac{1}{\left(4 \delta+2-2 \sqrt{1-\delta^{2}}\right)(1+\delta)}\right)<1+\delta .
\end{aligned}
$$

when $\delta$ is small enough. Therefore, any optimal solution $\left(x^{*}, w^{*}\right)$ must satisfy $x^{*} \notin \Gamma^{*}$. 


\subsubsection{Approximate Optimality Conditions}

The stopping criterion (3.13) implies $v\left(R O C_{\gamma}\right) \leq \varepsilon^{\prime} / \varepsilon$ : the tolerances provide the upper bound $\delta=\varepsilon^{\prime} / \varepsilon$ for the optimal value of (3.3). The values $\gamma$ for which this upper bound holds are strictly related to the following approximated problem

$$
\left(S R P_{\delta}\right) \quad \min \{d x+e w \mid x \in \Omega, w \in \Gamma, w x \geq 1+\delta\}
$$

which is obtained from (3.2) by perturbing the right-hand side of the nonconvex constraint. Our analysis does not require any regularity assumption on (3.15) and it is based on the following quantity

$$
\phi(\delta):=\inf \{d x+e w \mid x \in \Omega, w \in \Gamma, w x>1+\delta\} .
$$

Obviously, $\phi(\delta)$ may be greater than the optimal value of (3.15). Anyway, $\phi$ provides the right tool to disclose the connections between $\gamma,(3.13)$ and (3.15).

Proposition 3.3.1 Let $\delta \geq 0$. Then, the following statements are equivalent:

(i) $v\left(R O C_{\gamma}\right) \leq \delta$;

(ii) $R(\gamma) \subseteq\left\{(x, w) \in \mathbb{R}^{n} \times \mathbb{R}^{n} \mid w x \leq 1+\delta\right\}$;

(iii) $\gamma \leq \phi(\delta)$.

Proof. The equivalence between $(i)$ and (ii) follows immediately from the definition of $v\left(R O C_{\gamma}\right)$. Analogously, (ii) implies (iii) by the definition of $\phi(\gamma)$.

Suppose (ii) does not hold: there exist $(x, w) \in R(\gamma)$ such that $w x>1+\delta$. Take any $t \in(0,1)$ large enough to have $((1-t) \bar{x}+t x)((1-t) \bar{w}+t w)>1+\delta$. Since $(\bar{x}, \bar{w}) \in \Omega \times \Gamma$, the convexity of $\Omega \times \Gamma$ implies $((1-t) \bar{x}+t x,(1-t) \bar{w}+t w) \in \Omega \times \Gamma$; obviously $d((1-t) \bar{x}+t x)+e((1-t) \bar{w}+t w)<d x+e w \leq \gamma$. Therefore, $((1-t) \bar{x}+t x,(1-t) \bar{w}+t w)$ guarantees $\phi(\delta)<\gamma$ contradicting $(i i i)$.

Considering the optimal value of (3.15) as $\gamma$ in Proposition 3.3.1, we get that (ii) is a necessary optimality condition for (3.15). Furthermore, if the problem is regular (i.e. $\phi(\delta)$ is actually the optimal value), it is also sufficient. Choosing $\delta=0$, the known optimality conditions for (3.2) follows too. Therefore, inclusion 
(ii) can be considered as an approximate optimality condition for (3.2). As an immediate consequence of the proposition, we also have

$$
\phi(\delta)=\sup \left\{\gamma \mid R(\gamma) \subseteq\left\{(x, w) \in \mathbb{R}^{n} \times \mathbb{R}^{n} \mid w x \leq 1+\delta\right\}\right\}
$$

The stopping criterion $(i)$ guarantees approximate optimality and condition (iii) provides the adequate tool to evaluate the quality of the approximation. In fact, supposing (3.2) to be regular, i.e., $\gamma^{*}=\phi(0)$, we have that

$$
0 \leq \gamma-\gamma^{*} \leq \phi(\delta)-\gamma^{*}=\phi(\delta)-\phi(0)
$$

holds for any feasible value $\gamma$ which satisfies $(i)$. The following result guarantees that the approximation approaches the optimal value as $\delta$ goes to 0 .

Proposition 3.3.2 The value function $\phi$ is right-continuous at 0, i.e.,

$$
\lim _{\delta \downarrow} \phi(\delta)=\phi(0)
$$

Proof. Clearly $\phi$ is nonincreasing, that is $\phi\left(\delta^{1}\right) \geq \phi\left(\delta^{2}\right)$ whenever $\delta^{1} \geq \delta^{2} \geq 0$. As it is also bounded below by $\phi(0)$, there exist $\tilde{\gamma}=\lim _{\delta \downarrow 0} \phi(\delta)$ and $\tilde{\gamma} \geq \phi(0)$. Since $\tilde{\gamma} \leq \phi(\delta)$ for any $\delta>0$, Proposition 3.3.1 implies $v\left(R O C_{\tilde{\gamma}}\right) \leq \delta$ for any $\delta>0$. Since $v\left(R O C_{\tilde{\gamma}}\right)$ does not depend upon $\delta$, we get $v\left(R O C_{\tilde{\gamma}}\right) \leq 0$. Therefore, Proposition 3.3.1 guarantees $\tilde{\gamma} \leq \phi(0)$.

Although the approximation always converges to the optimal value, the rate of convergence may be less than linear as the Example 3.3.2 shows. Thus, regularity is not enough to achieve a linear rate of convergence. Additional assumptions on the problem are needed: the existence of an optimal solution with some particular properties guarantees the Lipschitz behaviour of $\phi$.

Example 3.3.2 Consider the (SRP) instance where

$$
\begin{aligned}
& \qquad \Omega=\left\{\left(x_{1}, x_{2}\right) \mid x_{1}^{2}+\left(x_{2}-1\right)^{2} \leq 4\right\} \\
& \Gamma=\left\{\left(w_{1}, w_{2}\right) \mid w_{1}+w_{2} \geq-0.5,2 w_{1}+w_{2} \leq 1,-2 w_{1}+w_{2} \leq 1,-w_{1}+w_{2} \geq-0.5\right\} \\
& \text { and } \bar{x}=\bar{w}=(0,0), d=(0,1), e=(1,0) \text {. The instance is depicted in Figure } \\
& \text { 3.1. From the figure, the optimal solution is } x^{*}=(-2,1) \text { and } w^{*}=(-1 / 2,0) \text { : }
\end{aligned}
$$



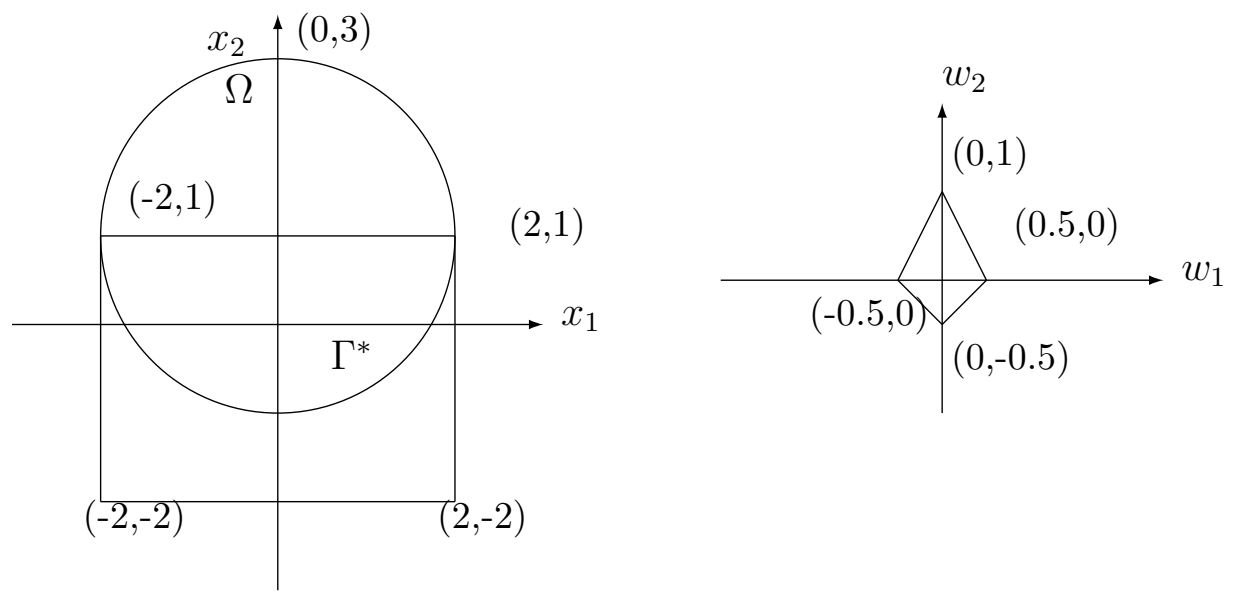

Figure 3.1: Data for the example: $\Omega, \Gamma^{*}$, and $\Gamma$

$\left(x^{*}, w^{*}\right)$ is feasible: $x^{*} w^{*}=1 ;\left(x^{*}, w^{*}\right)$ provides a lower bound of the instance: $d x^{*}=\min \left\{d x \mid x \in \Omega \backslash \operatorname{int} \Gamma^{*}\right\}, e w^{*}=\min \{e w \mid w \in \Gamma\}$ and $\min \{d x \mid x \in$ $\left.\Omega \backslash \operatorname{int} \Gamma^{*}\right\}+\min \{e w \mid w \in \Gamma\} \leq \min \{d x+e w \mid(x, w) \in \Omega \times \Gamma, w x \geq 1\}$. Therefore, the optimal value is $\gamma^{*}=1 / 2 ; T\left(\Omega, x^{*}\right) \nsubseteq T\left(\Gamma^{*}, x^{*}\right)$. Notice that this instance satisfies all standard assumptions: (3.5) and (3.6).

In order to prove the regularity condition of the instance, we construct a sequence of points $\{(x(\delta), w(\delta))\}$ such that $(x(\delta), w(\delta)) \in\{(x, w) \mid(x, w) \in$ $\Omega \times \Gamma, w x>1\}$ and $d x(\delta)+e w(\delta) \rightarrow \gamma^{*}$. Set

$$
x(\delta)=\left(-\sqrt{4-\delta^{2}}, 1+\delta\right) \in \Omega, w(\delta)=(-1 / 2+\delta, 2 \delta) \in \Gamma, \delta \leq 1 / 2 .
$$

Then $w(\delta) x(\delta)=(1 / 2-\delta) \sqrt{4-\delta^{2}}+2 \delta+2 \delta^{2}=: \chi(\delta)$ and $\chi(0)=1$. Moreover,

$$
\chi^{\prime}(\delta)=\frac{-(1-2 \delta) \delta}{2 \sqrt{4-\delta^{2}}}-\sqrt{4-\delta^{2}}+2+4 \delta=2-\sqrt{4-\delta^{2}}+\delta\left[4-\frac{1-2 \delta}{2 \sqrt{4-\delta^{2}}}\right]
$$

Therefore, $\chi^{\prime}(\delta)>0$ for $\delta>0$ small enough and $\chi^{\prime}(0)=0$. This implies that $\chi(\delta)>\chi(0)=1$ for all $\delta>0$ small enough, that is $w(\delta) x(\delta)>1$. It is easy to check that

$$
\lim _{\delta \rightarrow 0} d x(\delta)+e w(\delta)=\lim _{\delta \rightarrow 0} 1 / 2+2 \delta=1 / 2=\gamma^{*},
$$

this means that the instance is regular.

Then we prove that this instance doesn't satisfy the Lipschitz condition at 0 . Given any $\delta>0$, we have the following observations: any feasible solution $(x, w)$ to problem $\min \{d x+e w \mid(x, w) \in \Omega \times \Gamma, w x \geq 1+\delta\}$ must satisfy $w x \geq 1+\delta$ 
and thus $x \notin \operatorname{int} \Gamma_{\delta}^{*}$, this implies that $x_{2} \geq 1+\delta$ and $\left|x_{1}\right| \leq \sqrt{4-\delta^{2}}$. From Theorem 3.3.1 there always exists an optimal solution in the boundary of $\Omega$ and $\Gamma$; Figure 3.1 shows that the optimal solution $(x, w)$ can be found in $\left\{\left(x_{1}, x_{2}\right) \mid\right.$ $\left.x_{1}^{2}+x_{2}^{2}=4, x_{2} \geq 1, x_{1} \leq 0\right\}$ and $\left\{\left(w_{1}, w_{2}\right) \mid w_{2}=2 w_{1}+1, w_{2} \geq 0, w_{1} \leq 0\right\}$.

When $x_{2}=1+\delta$, we have

$$
x_{1} w_{1}+x_{2} w_{2}=x_{1} w_{1}+(1+\delta)\left(2 w_{1}+1\right) \geq 1+\delta,
$$

Therefore, $\frac{w_{1} x_{1}}{1+\delta}+2 w_{1} \geq 0$. From $\left|x_{1}\right| \leq \sqrt{4-\delta^{2}}$ we know that $2+\frac{x_{1}}{1+\delta}>0$ and thus $w_{1} \geq 0$. Therefore,

$$
d x+e w=x_{2}+w_{1} \geq 1+\delta .
$$

When $x_{2}>1+\delta$, from $(3.17)$ we have

$$
w_{1}=\frac{1+\delta-x_{2}}{x_{1}+2 x_{2}}, w_{2}=\frac{x_{1}+2+2 \delta}{x_{1}+2 x_{2}},
$$

then

Therefore,

$$
\varphi_{x_{1}}\left(x_{2}\right)=d x+e w=x_{2}+\frac{1+\delta-x_{2}}{x_{1}+2 x_{2}}
$$

$$
\varphi_{x_{1}}^{\prime}\left(x_{2}\right)=0 \Rightarrow=1-\frac{1}{x_{1}+2 x_{2}}-\frac{2\left(1+\delta-x_{2}\right)}{\left(x_{1}+2 x_{2}\right)^{2}}=1-\frac{x_{1}+2+2 \delta}{\left(x_{1}+2 x_{2}\right)^{2}}=0,
$$

then $x_{2}^{1}=\frac{-x_{1}-\sqrt{x_{1}+2+2 \delta}}{2}$ and $x_{2}^{2}=\frac{-x_{1}+\sqrt{x_{1}+2+2 \delta}}{2} .1+\delta<x_{2}^{1} \leq 3 \Rightarrow\left|x_{1}\right|>$ $\sqrt{4-\delta^{2}}$, a contradiction; $1+\delta<x_{2}^{2} \leq 3 \Rightarrow x_{1}<-1-2 \delta$. Then

$$
\min _{x_{2} \in(1+\delta, 3]} \varphi_{x_{1}}\left(x_{2}\right)= \begin{cases}\varphi_{x_{1}}\left(x_{2}^{2}\right) & \text { if }-\sqrt{4-\delta^{2}} \leq x_{1}<-1-2 \delta \\ \varphi_{x_{1}}(1+\delta)=1+\delta & \text { if }-1-2 \delta \leq x_{1} \leq \sqrt{4-\delta^{2}}\end{cases}
$$

Then we have

$$
\varphi_{x_{1}}\left(x_{2}^{2}\right)=x_{2}^{1}+\frac{1+\delta-x_{2}}{x_{1}+2 x_{2}}=\sqrt{x_{1}+2+2 \delta}-\frac{1+x_{1}}{2}=\psi\left(x^{1}\right) .
$$

Moreover, $\psi^{\prime}\left(x^{1}\right)=\frac{1}{2 \sqrt{x_{1}+2+2 \delta}}-\frac{1}{2}<0$ since $x_{1}<-1-2 \delta$, this implies that

$$
\inf _{-\sqrt{4-\delta^{2}} \leq x_{1}<-1-2 \delta} \psi\left(x^{1}\right) \geq \min _{-2 \leq x_{1}<-1-2 \delta} \psi\left(x^{1}\right)=\psi(-2)=\frac{1}{2}+\sqrt{2 \delta} .
$$

Therefore, by the regularity condition we have

$$
\lim _{\delta \rightarrow 0} \frac{\phi(\delta)-\phi(0)}{\delta} \geq \lim _{\delta \rightarrow 0} \frac{\min \left\{1+\delta, \frac{1}{2}+\sqrt{2 \delta}\right\}-\gamma^{*}}{\delta}=\lim _{\delta \rightarrow 0} \frac{\sqrt{2 \delta}}{\delta}=+\infty .
$$

Then the instance doesn't satisfy the Lipschitz Condition at 0 . 

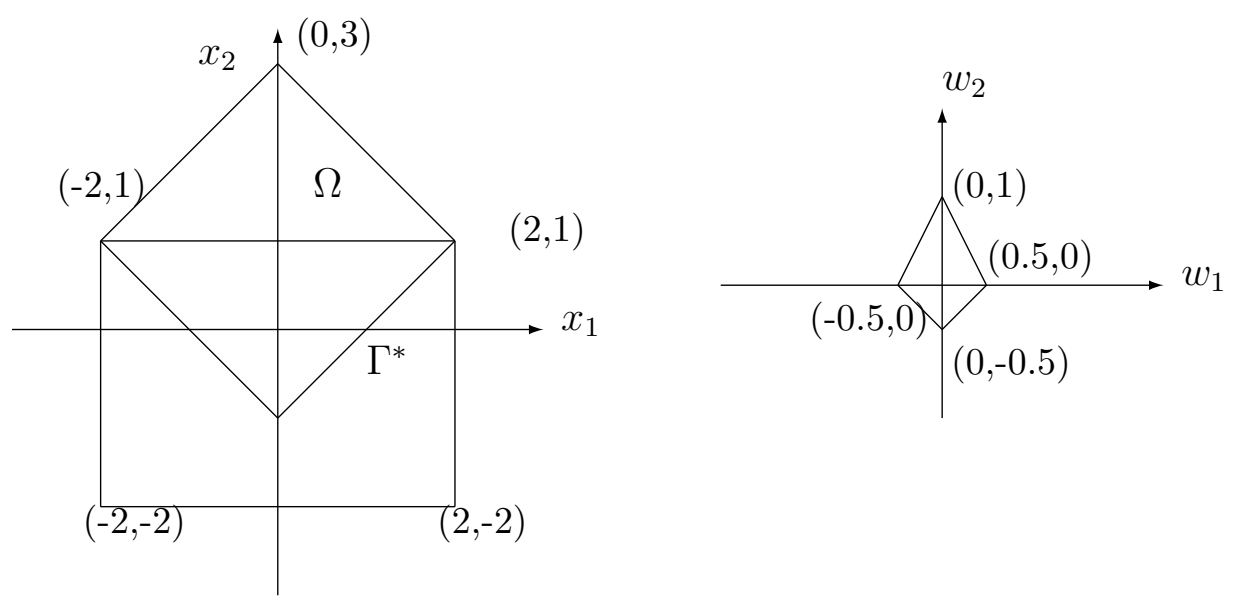

Figure 3.2: Data for the example: $\Omega, \Gamma^{*}$, and $\Gamma$

Example 3.3.2 contradicts Theorem 2.2.1, which means that $T\left(\Omega, x^{*}\right) \nsubseteq$ $T\left(\Gamma^{*}, x^{*}\right)$ can not be used to judge the Lipschitz property of problem (SRP). In fact, $T\left(\Omega, x^{*}\right) \nsubseteq T\left(\Gamma^{*}, x^{*}\right)$ is also not enough to imply regularity of (SRP) even when $\Gamma^{*}$ is a polyhedron, i.e., Theorem 2.2.2 doesn't hold in (SRP).

Example 3.3.3 Consider the (SRP) instance where

$$
\Omega=\left\{\left(x_{1}, x_{2}\right) \mid x_{1}-1 \leq x_{2} \leq x_{1}+3,-x_{1}-1 \leq x_{2} \leq-x_{1}+3\right\}
$$

$\Gamma=\left\{\left(w_{1}, w_{2}\right) \mid w_{1}+w_{2} \geq-0.5,2 w_{1}+w_{2} \leq 1,-2 w_{1}+w_{2} \leq 1,-w_{1}+w_{2} \geq-0.5\right\}$ and $\bar{x}=\bar{w}=(0,0), d=(0,1), e=(1,0)$. The instance is depicted in Figure 3.2. From the Figure, it is clear that the optimal solution is $x^{*}=(-2,1)$ and $w^{*}=(-1 / 2,0):\left(x^{*}, w^{*}\right)$ is feasible: $x^{*} w^{*}=1, d x^{*}+e w^{*}$ provides a lower bound of the optimal value: $d x^{*}=\min \left\{d x \mid x \in \Omega \backslash\right.$ int $\left.\Gamma^{*}\right\}, e w^{*}=\min \{e w \mid w \in \Gamma\}$ and $\min \left\{d x \mid x \in \Omega \backslash \operatorname{int} \Gamma^{*}\right\}+\min \{e w \mid w \in \Gamma\} \leq \min \{d x+e w \mid(x, w) \in$ $\Omega \times \Gamma, w x \geq 1\}$. Therefore, the optimal value is $\gamma^{*}=1 / 2 ; T\left(\Omega, x^{*}\right) \nsubseteq T\left(\Gamma^{*}, x^{*}\right)$. Notice that this instance satisfies all standard assumptions: (3.5) and (3.6). For any problem

$$
\varphi(\delta)=\min \{d x+e w \mid(x, w) \in \Omega \times \Gamma, w x \geq 1+\delta\},
$$

set the optimal solution $x=\left(-2+\lambda_{1}, 1+\lambda_{1}\right)$ and $w=\left(-0.5+\lambda_{2}, 2 \lambda_{2}\right)$; from the figure, it is clear that $\lambda_{2}>0$, for otherwise $w \in \operatorname{int} \Omega_{\delta}^{*}$ for all $\delta>0$. Then 
we get that $w x=\left(-2+\lambda_{1}\right)\left(-0.5+\lambda_{2}\right)+\left(1+\lambda_{1}\right) 2 \lambda_{2}=1+\delta$, which implies that $\lambda_{2}\left(3 \lambda_{1}-0.5\right)=\delta$. Since $\lambda_{2}>0$, we get that $\lambda_{1}>1 / 6$. Therefore,

$$
\phi(\delta) \geq \varphi(\delta)=1 / 2+\lambda_{1}+\lambda_{2}>2 / 3
$$

for all $\delta>0$, that is

$$
\inf _{\delta>0} \phi(\delta)=\inf \{d x+e w \mid(x, w) \in \Omega \times \Gamma, w x>1\}>2 / 3>1 / 2=\gamma^{*} .
$$

Therefore, the instance is not regular.

Proposition 3.3.3 If there exists an optimal solution $\left(x^{*}, w^{*}\right)$ to (3.2) such that (2.10) or

$$
\left\{w^{*}+\lambda u \mid \lambda>0\right\} \cap \Gamma \neq \emptyset \text { and } x^{*} u>0
$$

for some direction $u \in \mathbb{R}^{n}$, then the value function $\phi$ is locally Lipschitz at 0 , i.e., there exist $L>0$ and $\bar{\delta}>0$ such that

$$
\phi(\delta)-\phi(0) \leq L \delta \quad \forall \delta \in[0, \bar{\delta}]
$$

Proof. It is enough to prove condition (2.10), as the other case is symmetric. Let $\bar{\lambda}>0$ be such that $x^{*}+\bar{\lambda} u \in \Omega$; the convexity of $\Omega$ implies $x(\lambda):=x^{*}+\lambda u \in \Omega$ for any $\lambda \in[0, \bar{\lambda}]$; furthermore, $w^{*}\left(x^{*}+\lambda u\right)=1+\lambda w^{*} u>1$ if $\lambda>0$. Thus, the sequence $\left(x(\lambda), w^{*}\right)$ shows that the regularity condition (3.8) holds. Therefore, we have $\phi(0)=d x^{*}+e w^{*}$.

Chosen $\bar{\delta}:=\left(w^{*} u / 2\right) \bar{\lambda}$, let us consider $y(\delta):=x\left(2 \delta / w^{*} x\right)$ for any $\delta \in(0, \bar{\delta}]$ : we have $y(\delta) \in \Omega$ and

$$
w^{*} y(\delta)=w^{*} x^{*}+\left(2 \delta / w^{*} u\right) w^{*} u=1+2 \delta>1+\delta,
$$

where the last equality holds since optimality implies $w^{*} x^{*}=1$. Therefore, $\left(y(\delta), w^{*}\right)$ provides an upper bound for $\phi(\delta)$, i.e. $\phi(\delta) \leq d y(\delta)+e w^{*}$. Finally, we get

$$
\phi(\delta)-\phi(0) \leq d y(\delta)+e w^{*}-d x^{*}-e w^{*}=\left(2 d u / w^{*} u\right) \delta
$$

(2.10) and (3.18) imply $w^{*} \notin \Omega^{*}$ and $w^{*} \notin \Gamma^{*}$, respectively. This leads to the following corollary.

Corollary 3.3.1 If there exists an optimal solution $\left(x^{*}, w^{*}\right)$ of problem (SRP) such that $x^{*} \notin \Gamma^{*}$ or $w^{*} \notin \Omega^{*}$, then the value function $\phi$ is locally Lipschitz at 0 . 


\subsection{Conditions and Algorithms}

In this section, we present algorithms which approximately solve (SRP) problem if an approximated oracle $\Theta$ is available. We first establish a hierarchy of abstract conditions ensuring convergence, then, for each set of conditions we propose actual implementable procedures which realize it.

\subsubsection{General Convergence Conditions}

All the algorithms follow the generic cutting plane scheme sketched in the previous section. More in details, a non increasing sequence of feasible values $\left\{\gamma^{k}\right\}$ is produced, and the oracle $\Theta$ is called for each $\gamma^{k}$, thereby producing either a value $l^{k}$ such that condition (3.13) holds, or points $z^{k}$ and $v^{k}$ satisfying conditions (3.14). By repeatedly calling the oracle, if necessary, we can construct a procedure which either proves that $\gamma^{k}$ satisfies condition (3.13), or produces a better feasible value $\gamma^{k+1}<\gamma^{k}$. In the latter case, $\gamma^{k+1}$ is associated to (produced by) points $x^{k}$ and $w^{k}$ such that

$$
x^{k} \in \Omega, w^{k} \in \Gamma, w^{k} x^{k}=1
$$

and $\gamma^{k+1}=d x^{k}+e w^{k}$. The rationale for (3.19) is that, due to feasibility and $w^{*} x^{*}=1$, any optimal solution must satisfy this condition.

With the above notation, we can introduce the prototype Algorithm 5.

\section{Algorithm 5 Prototype Algorithm}

0. $\gamma^{1}=+\infty ; k=1$;

1. If optimality condition (3.7) holds, then $\gamma^{k}$ is the optimal value and stop;

2. Select $\left(x^{k}, w^{k}\right)$ satisfying (3.19) such that $\gamma^{k+1}=d x^{k}+e w^{k}<\gamma^{k}$;

set $k=k+1$; goto 1 .

Clearly, if at Step 0 (initialization) some feasible pair $\left(x^{0}, w^{0}\right)$ is known, one can alternatively set $\gamma^{1}=d x^{0}+e w^{0}$. An important feature of Algorithm 5 is that $\left\{\gamma^{k}\right\}$ is a decreasing sequence bounded below:

$$
\bar{\gamma}<\gamma^{*} \leq \gamma^{\infty}=\lim _{k \rightarrow \infty} \gamma^{k}<\cdots<\gamma^{k}<\gamma^{k-1}<\cdots<\gamma^{1}
$$


Therefore, $\left\{R\left(\gamma^{k}\right)\right\}$ is a "non-increasing sequence", i.e.,

$$
R\left(\gamma^{\infty}\right) \subseteq \cdots \subseteq R\left(\gamma^{k+1}\right) \subseteq R\left(\gamma^{k}\right) \subseteq \cdots \subseteq R\left(\gamma^{1}\right)
$$

Obviously, Algorithm 5 is too general to deduce any meaningful property; some more has to be said about two things:

1. how exactly optimality condition (3.7) is checked,

2. how $\left(x^{k}, w^{k}\right)$ such that $d x^{k}+e w^{k}<\gamma^{k}$ is selected once one knows that (3.7) is not satisfied.

Clearly, the two points are strictly interwoven, in that finding $\left(x^{k}, w^{k}\right)$ such that $d x^{k}+e w^{k}<\gamma^{k}$ immediately proves that $\gamma^{k}$ is not optimal. Vice versa, assume that we have any constructive procedure that eventually produces a point $\left(z^{k}, v^{k}\right) \in R\left(\gamma^{k}\right)$ such that $v^{k} z^{k}>1$ when $\gamma^{k}$ is not optimal. Pick $\left(x^{k}, w^{k}\right)$ from the intersection between $\{(x, w) \mid w x=1\}$ and the segment with $(\bar{x}, \bar{w})$ and $\left(z^{k}, v^{k}\right)$ as the end points: clearly, $\left(x^{k}, w^{k}\right) \in R\left(\gamma^{k}\right)$ and $d x^{k}+e w^{k}<d z^{k}+e v^{k} \leq$ $\gamma^{k}$. Then, a first question is if such a method provides a convergent algorithm; not surprisingly, without further qualification the answer is negative ([10, Example 4.1]).

Thus some care is needed in choosing the sequences $x^{k}$ and $w^{k}$. Actually, our "more implementable" approximate optimality condition based on (2.1) indicates that other two sequences $z^{k}$ and $v^{k}$, which are "to $x^{k}$ and $w^{k}$ ", should be taken into account as well. In fact, we propose condition (2.17)and the following general assumptions under which convergence can be proved:

$$
v^{k} z^{k}-1 \geq \varepsilon \max \left\{v z-1 \mid(z, v) \in R\left(\gamma^{k}\right)\right\}
$$

where $\varepsilon \in(0,1)$. Condition (3.20) basically says that $v^{k}$ and $z^{k}$ must be produced by some process attempting to solve the non-convex problem $\left(R O C_{\gamma^{k}}\right)(\mathrm{cf} .(3.3))$, although the process may be "terminated early" due to the optimality tolerance $\varepsilon$. Condition (2.17) rather requires the two sequences to be asymptotically jointly feasible, and, as we will see, there are several different implementable ways for ensuring that this holds. Anyway, as far as abstract conditions go, (2.17) and (3.20) are already sufficient to guarantee convergence to the optimal value.

Proposition 3.4.1 If (2.17) and (3.20) hold, then the sequence of feasible values $\left\{\gamma^{k}\right\}$ in Algorithm 5 converges to the optimal value $\gamma^{*}$. 
Proof. Since each $\gamma^{k}$ is a feasible value, we have $\gamma^{*} \leq \gamma^{\infty}$, i.e., $\gamma^{\infty}$ is a feasible value, too. Hence, (3.20) implies that

$$
v^{k} z^{k}-1 \geq \varepsilon \max \left\{v z-1 \mid(z, v) \in R\left(\gamma^{\infty}\right)\right\}
$$

for all $k$. Taking the limit, (2.17) implies

$$
\max \left\{v z-1 \mid(z, v) \in R\left(\gamma^{\infty}\right)\right\} \leq 0,
$$

and therefore $\gamma^{\infty}$ is the optimal value.

When developing a "concrete" algorithm for (SRP), the abstract condition (2.17) can not be directly imposed on the sequences $\left\{z^{k}\right\}$ and $\left\{v^{k}\right\}$. In fact, these are the results of "complex" optimization process, i.e. approximately solving (3.3), upon which we want to impose as few conditions as possible, in order to leave as much freedom as possible to different implementations of this critical task. Therefore, we seek alternative ways for obtaining condition (2.17). However, given $z^{k}$ and $v^{k}$ as produced by the oracle we have full control on how $x^{k}$ and $w^{k}$ are constructed, provided that (3.19) is satisfied; we can use this to enforce (2.17).

Lemma 2.3.1 guarantees that either (2.18) or (2.19) imply (2.17), we can define the two (highly symmetric) sets of conditions which, separately, guarantee convergence of Algorithm 5:

$$
B_{3} \equiv(3.20) \text { and }(2.18) \quad B_{4} \equiv(3.20) \text { and }(2.19)
$$

Though they look highly symmetric to each other, we will show that $B_{3}$ and $B_{4}$ are by no means equivalent. In fact, we will propose several different sets of conditions which imply one of them, and develop implementable sub-procedures that attain these conditions, thereby defining three different implementable algorithms.

\subsubsection{The Outer Approximation Machinery}

As anticipated in $\S 3.3 .1$, one important way to make (3.3) more tractable is to replace $\Omega$ and $\Gamma$ by two "simpler" convex sets $Q$ and $S$ such that $\Gamma \subseteq Q$ and $\Omega \subseteq S$; of course, this requires some appropriate machinery to update $S$ and $Q$ 
in order to make them "good enough" approximations of $\Omega$ and $\Gamma$. Convexity of both sets allows to rely on cutting plane procedures based on standard separation tools, for which powerful results like Theorem 2.3.1 are known. Furthermore, Theorem 2.3.1 justifies for the classical cutting-plane Sub-procedure 2.

\subsubsection{A Generic Outer Approximation Sub-Procedure}

We can now define a generic outer approximation procedure which, only provided with an approximate oracle $\Theta$, allows implementations of Algorithm 5 which attain the sufficient convergence conditions introduced in $\S 3.4 .1$. We call Subprocedure 6 a "generic" outer approximation procedure because it depends on two parameters: $\Psi$ and $\Upsilon$. In this paragraph we will describe the properties of the Sub-Procedure independent on the specific choice of $\Psi$ and $\Upsilon$; later on we will show several different possible choices for $\Psi$ and $\Upsilon$, leading to different implementable algorithms for (SRP). In the Sub-procedure, given a direction $y \in \mathbb{R}^{n}$ we will often use the normalized direction $N(y)=y /\|y\|$ if $y \neq 0$, $N(y)=0$ if $y=0$.

Some remarks on Sub-procedure 6 are in order:

- The condition

$$
x^{i} \in\left[\bar{x}, z^{i}\right] \quad \text { and } \quad w^{i} \in\left[\bar{w}, v^{i}\right]
$$

at step 6 guarantees $x^{i} \in \Omega$ and $w^{i} \in \Gamma$ for all $\left(z^{i}, v^{i}\right)$ produced in step 4. The conditions that guarantee the existence of $\left(x^{i}, w^{i}\right)$ satisfying (3.21) and different combinations of $\Psi$ and $\Upsilon$ will be discussed in details later on, that is why we need not consider the non-existence case of $\left(x^{i}, w^{i}\right)$ in step 6.

- From conditions (3.9) and (3.10), $\Omega$ and $\Gamma$ are included in $S^{i}$ and $Q^{i}$, respectively, for $i=1$. The cutting-plane Sub-procedure 2 ensures this is still true for any $i$, i.e., we get "non-increasing" sequences $\left\{S^{i}\right\}$ and $\left\{Q^{i}\right\}$

$$
\begin{aligned}
& \Omega \subseteq \cdots \subseteq S^{i+1} \subseteq S^{i} \subseteq \cdots \subseteq S^{1} \\
& \Gamma \subseteq \cdots \subseteq Q^{i+1} \subseteq Q^{i} \subseteq \cdots \subseteq Q^{1} .
\end{aligned}
$$

- The choice of $\left(z^{i}, v^{i}\right)$ at step 4 guarantees that

$$
d z^{i}+e v^{i} \leq \max \left\{d \bar{z}^{i}+e \bar{v}^{i} ; d \bar{x}+e \bar{w}\right\} \leq \gamma
$$




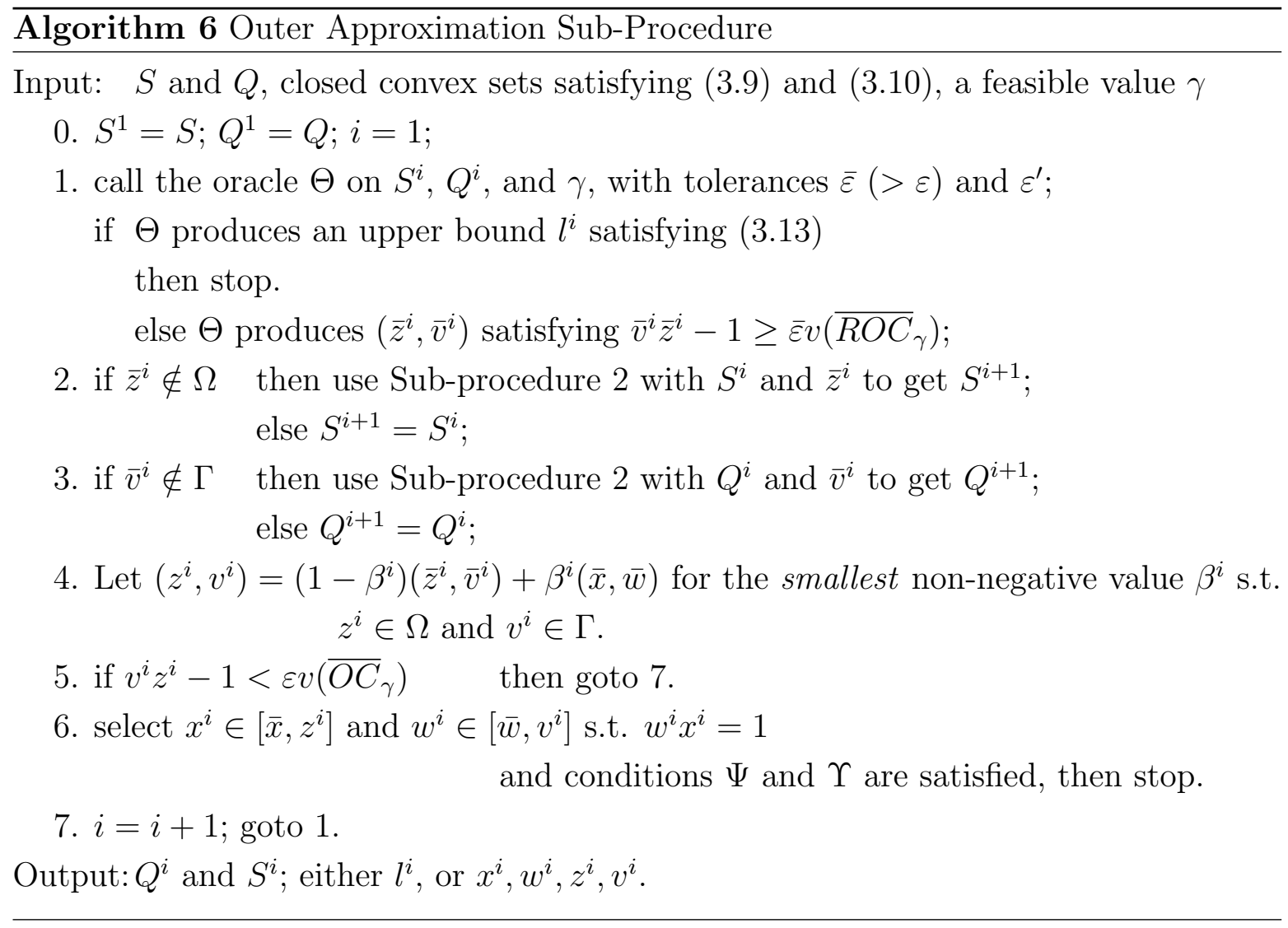

i.e., $z^{i}$ and $v^{i}$ are also feasible for the maximization problem (approximately) solved by the oracle. Note that the step $\alpha^{i}$ can be 0 , i.e. when $\left(\bar{z}^{i}, \bar{v}^{i}\right) \in \Omega \times \Gamma$.

- The condition " $v^{i} z^{i}-1<\varepsilon v\left(\overline{R O C}_{\gamma}\right)$ " at step 5 may be difficult the check directly, as the value of $v\left(\overline{R O C}_{\gamma}\right.$ ) is not known (although a suitable upper bound must be computed by $\Theta$ in order to be able to guarantee that (3.14) holds). A stronger condition that can be surely checked is $v^{i} z^{i}-1 \geq$ $\left(\bar{v}^{i} \bar{z}^{i}-1\right)(\varepsilon / \bar{\varepsilon})$; if that holds, then we are guaranteed that $\left(z^{i}, v^{i}\right)$ satisfies (3.14) and the algorithm can proceed with step 5 , otherwise it loops.

- If the algorithm loops at step 5 , then at least one among $Q^{i} \neq Q^{i+1}$ and $S^{i} \neq S^{i+1}$ holds. In fact, if $\bar{z}^{i} \in \Omega$ and $\bar{v}^{i} \in \Gamma$ then $z^{i}=\bar{z}^{i}$ and $v^{i}=\bar{v}^{i}$, so the condition at step 5 cannot be true.

- In the algorithm, we are forced to require to the oracle $\Theta$ a "stricter" 
tolerance $\bar{\varepsilon}$ in order to be able to guarantee convergence to a solution that is optimal only to within $\delta=\varepsilon^{\prime} / \varepsilon$ for the "looser" tolerance $\varepsilon<\bar{\varepsilon}$; the exact role of this assumption will be discussed in details later on. However, nothing is required to the ratio $\varepsilon / \bar{\varepsilon}$ except being strictly smaller than one, so the two tolerances can be taken arbitrarily close to each other.

From the previous discussion, it is at least proven that the Sub-procedure will never repeat the same iterates; if it does not stop, then at least one among $S^{i} \backslash\left\{\bar{z}^{i}\right\} \supset S^{i+1}$ and $Q^{i} \backslash\left\{\bar{v}^{i}\right\} \supset Q^{i+1}$ hold, so at least one among $\bar{z}^{i+1} \neq \bar{z}^{i}$ and $\bar{v}^{i+1} \neq \bar{v}^{i}$ hold. We now prove some basic properties of Sub-procedure 6 that lead to finite termination under $\varepsilon^{\prime}>0$, which is independent on the choice of $\Psi$ and $\Upsilon$.

Lemma 3.4.1 If Sub-procedure 6 never ends, then all the cluster points of $\left\{\bar{z}^{i}\right\}$ and $\left\{\bar{v}^{i}\right\}$ belong to $\Omega$ and $\Gamma$, respectively.

Proof. Consider $\left\{\bar{z}^{i}\right\}$ : either $\bar{z}^{i} \in \Omega$ for all large enough $i$, and therefore the thesis is proved by closeness of $\Omega$, or $\bar{z}^{i} \in S^{i} \backslash \Omega$ for infinitely many times. In the latter case, the hypotheses of Theorem 2.3.1 are satisfied, so again all the cluster points of $\left\{\bar{z}^{i}\right\}$ belong to $\Omega$. The same line of reasoning works for $\left\{\bar{v}^{i}\right\}$.

In the following development, it will be crucial to ensure that the sequences $\left\{\bar{z}^{i}\right\}$ and $\left\{\bar{v}^{i}\right\}$ do indeed have cluster points. This is easily done: both $\Omega$ and $\Gamma$ are assumed to be compact; therefore, it is natural to assume

$$
\left\{\bar{z}^{i}\right\} \text { and }\left\{\bar{v}^{i}\right\} \text { are bounded }
$$

which, in view of (3.22) and (3.23), hold e.g. if every $S^{k}$ is contained into a given compact set $S^{0}(\supseteq \Omega)$, and every $Q^{k}$ is contained into a given compact set $Q^{0}(\supseteq \Gamma)$. We therefore assume (3.25) to hold in all the following development. In fact (2.23) holds since they belong to bounded sets ( $\Omega$ and $\Gamma$, respectively), which also implies the boundedness of $\left\{x^{i}\right\}$ and $\left\{w^{i}\right\}$. Proving finiteness of the sub-procedure requires knowledge of properties of the sequences of points, such as the relationship between the cluster points of the "original" sequences $\left(\bar{z}^{i}, \bar{v}^{i}\right)$ and those of the "modified" sequences $\left(z^{i}, v^{i}\right)$. 
Lemma 3.4.2 If infinitely many iterates $z^{i}$ and $v^{i}$ are produced, then there is a one-to-one correspondence between the cluster points of $\left\{\bar{z}^{i}\right\}\left[\left\{\bar{v}^{i}\right\}\right]$ and those of $\left\{z^{i}\right\}\left[\left\{v^{i}\right\}\right]$.

Proof. It suffices to prove that 0 is the only cluster point of $\left\{\beta^{i}\right\}$. Assume by contradiction that $\beta^{i} \rightarrow \bar{\beta} \in(0,1]$. By taking subsequences if necessary, let $\left(\bar{z}^{i}, \bar{v}^{i}\right) \rightarrow(\bar{z}, \bar{v})$, then we have $\left(z^{i}, v^{i}\right) \rightarrow(\hat{z}, \hat{v})=(1-\bar{\beta})(\bar{z}, \bar{v})+\bar{\beta}(\bar{x}, \bar{w})$ for $\left(z^{i}, v^{i}\right)=\beta^{i}(\bar{x}, \bar{w})+\left(1-\beta^{i}\right)\left(\bar{z}^{i}, \bar{v}^{i}\right)$. Since $\bar{\beta}>0,(\bar{x}, \bar{w}) \in \operatorname{int} \Omega \times$ int $\Gamma$ and $(\bar{z}, \bar{v}) \in \Omega \times \Gamma$, we then get that $(\hat{z}, \hat{v}) \in \operatorname{int} \Omega \times \operatorname{int} \Gamma$.

Therefore, for sufficiently large $i$, we have $\left(z^{i}, v^{i}\right)=\beta^{i}(\bar{x}, \bar{w})+\left(1-\beta^{i}\right)\left(\bar{z}^{i}, \bar{v}^{i}\right) \in$ int $\Omega \times$ int $\Gamma$ and thus the segment with $\left(z^{i}, v^{i}\right)$ and $\left(\bar{z}^{i}, \bar{v}^{i}\right)$ as end points has nonempty intersection with the set int $\Omega \times$ int $\Gamma$. This contradicts the assumption that $\beta^{i}$ is the smallest non-negative value such that $\beta^{i}(\bar{x}, \bar{w})+\left(1-\beta^{i}\right)\left(\bar{z}^{i}, \bar{v}^{i}\right) \in$ $\Omega \times \Gamma$.

If the Sub-procedure never ends, then $\left(z^{i}, v^{i}\right)$ is defined and $v^{i} z^{i}-1<$ $\varepsilon v\left(\overline{R O C}_{\gamma}\right)$ for infinitely many times. We are ready to show that this is not true.

Proposition 3.4.2 If $\varepsilon^{\prime}>0$, then Sub-procedure 6 stops after a finite number of iterations.

Proof. Assume the contrary holds; one has " $v^{i} z^{i}-1<\varepsilon v\left(\overline{R O C}_{\gamma}\right)$ " for infinite number of $i$, that is

$$
\left(v^{i} z^{i}-1\right) / \varepsilon<v\left(\overline{R O C}_{\gamma}\right) \leq\left(\bar{v}^{i} \bar{z}^{i}-1\right) / \bar{\varepsilon}
$$

infinitely many times. But a common cluster point $(\bar{z}, \bar{v})$ of $\left\{\left(z^{i}, v^{i}\right)\right\}$ and $\left\{\left(\bar{z}^{i}, \bar{v}^{i}\right)\right\}$ exists by Lemma 3.4.2, (3.25) and (2.23), for which by continuity $\bar{v} \bar{z}-1 \geq \varepsilon^{\prime}>0$ and

$$
1=\frac{\bar{v} \bar{z}-1}{\bar{v} \bar{z}-1} \leq \frac{\varepsilon}{\bar{\varepsilon}}<1
$$

a contradiction.

The above proof shows the need for requiring $\varepsilon^{\prime}>0$, since for $\varepsilon^{\prime}=0$ the Subprocedure may never stop. In other words the Sub-procedure can not finitely prove that the optimal value is optimal; this is why it is relevant to clarify 
the relationship between approximated optimal values and the optimal value. Furthermore, the proof also shows that requiring the "tighter" tolerance $\bar{\varepsilon}$ on $\left(\bar{z}^{i}, \bar{v}^{i}\right)$ is needed in order to ensure that the "looser" tolerance $\varepsilon$ is attained on the modified iterates $\left(z^{i}, v^{i}\right)$, and therefore the sub-procedure can have finite termination.

If Sub-procedure 6 stops at step 1, then $\gamma$ is approximately optimal with positive tolerances $\bar{\varepsilon}$ and $\varepsilon^{\prime}$; if it stops at step 6 , the existence and convergence properties of $\left(x^{i}, w^{i}\right)$ would depend on the exact choice of combinations of $\Psi$ and $\Upsilon$. Detailed discussion on different combinations and the corresponding convergence proofs will be given in the following sections.

We finish this section with a further result that will greatly simplify the analysis of the convergence of the following algorithms. For several of them, it is necessary to impose a further condition (other than $\gamma \geq \gamma^{*}$ ) on the initial value, i.e.

$$
\begin{aligned}
\gamma & \leq \min \left\{\gamma_{\bar{x}}, \gamma_{\bar{w}}\right\}, \text { where } \\
\gamma_{\bar{x}} & =d \bar{x}+\min \{e w \mid w \in \Gamma, w \bar{x} \geq 1\} \\
\gamma_{\bar{w}} & =e \bar{w}+\min \{d x \mid x \in \Omega, \bar{w} x \geq 1\}
\end{aligned}
$$

Note that $\gamma_{\bar{x}}$ and $\gamma_{\bar{w}}$ are the optimal values of two convex problems, hence "easily" obtained; furthermore, since $\gamma^{k}$ is decreasing, if (3.26) holds at the first iteration then it automatically holds at all subsequent ones, thus the problems only need to be solved once. However, it has to be remarked that either one (and even both) can be $+\infty$, as there is no guarantee that the corresponding feasible regions are nonempty; this is for instance surely the case if $(\bar{x}, \bar{w})=(0,0)$, which can always be assumed w.l.o.g. in the (CDC) case.

Lemma 3.4.3 Under condition (3.26),

$$
\begin{aligned}
& e v^{i} \geq e \bar{w} \quad \Rightarrow \quad \bar{w} z^{i} \leq 1 \\
& d z^{i} \geq d \bar{x} \quad \Rightarrow \quad v^{i} \bar{x} \leq 1
\end{aligned}
$$

Proof. We only prove (3.27), as the other case is symmetric. Assume by contradiction $e v^{i} \geq e \bar{w}$ but $\bar{w} z^{i}>1$; then, since $\bar{w} \bar{x}<1$, by the mean value theorem there exists some $\tilde{x}$ such that

$$
\tilde{x} \in\left(\bar{x}, z^{i}\right) \text { and } \bar{w} \tilde{x}=1 \text {. }
$$


Hence, by the definition of $\gamma_{\bar{w}}$

$$
d \bar{x}+e \bar{w}<\gamma \leq \gamma_{\bar{w}} \leq d \tilde{x}+e \bar{w} .
$$

Therefore, $d \tilde{x}>d \bar{x}$, finally yielding $d \bar{x}<d \tilde{x}<d z^{i}$; we therefore have

$$
\gamma_{\bar{w}} \leq d \tilde{x}+e \bar{w}<d z^{i}+e v^{i} \leq \gamma \leq \gamma_{\bar{w}}
$$

i.e. a contradiction.

\subsubsection{Implementable algorithms}

Sub-procedure 6 can be used to define implementable versions of the Prototype Algorithm 5, as follows:

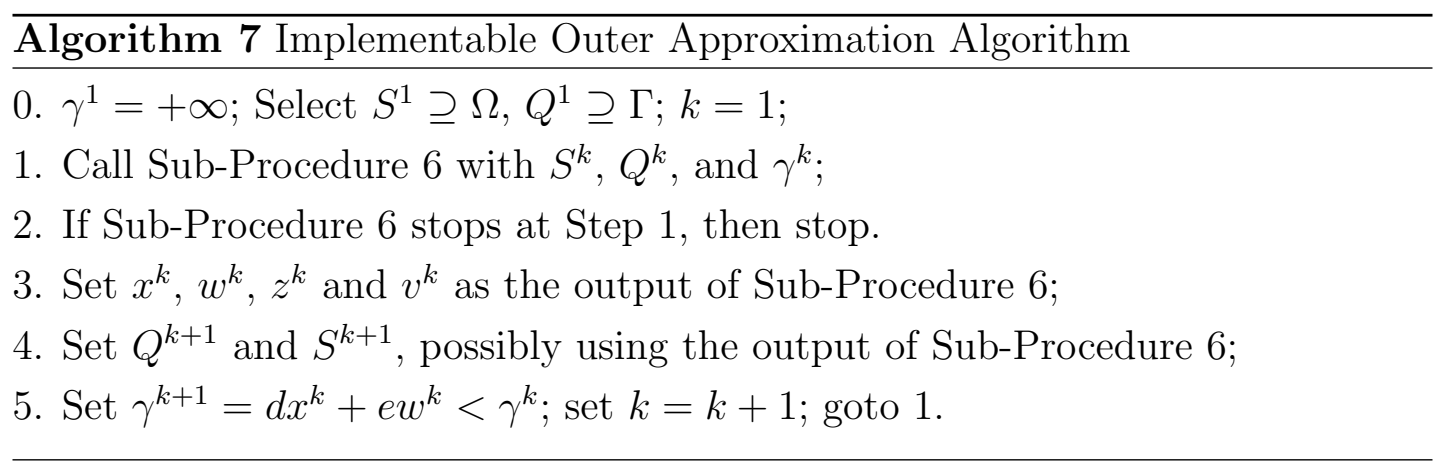

Some remarks on Algorithm 7 are in order:

- clearly, due to (3.14), (3.22) and (3.23), condition (3.20) is always satisfied by all possible variants of the algorithm, i.e., irrespective of $\Psi$ and $\Upsilon$;

- at Step 4 , the obvious possibility for $Q^{k+1}$ and $S^{k+1}$ is to set them as the $Q^{i}$ and $S^{i}$ produced by Sub-Procedure 6; however, this leads to accumulation in $Q^{k}$ and $S^{k}$ of all cutting planes generated along the iterates, and therefore possibly to "large" descriptions of $Q^{k}$ and $S^{k}$, so one may want to "purge" some of the accumulated cutting planes, which can always be done since only (3.9) and (3.10) need be satisfied;

In order for the Algorithm 7 to work, at least one among conditions $B_{3}$ and $B_{4}$ must hold. This require appropriate conditions on the way in which $x^{k}$ and $w^{k}$ 
are constructed out of $z^{k}$ and $v^{k}$. It will be convenient to introduce the following notation:

$$
z^{k}=x^{k}+\lambda_{1}^{k} y^{k}, \quad v^{k}=w^{k}+\lambda_{2}^{k} u^{k} .
$$

That is, $x^{k}$ and $w^{k}$ are obtained from $z^{k}$ and $v^{k}$ moving along the directions $-y^{k}$ and $-u^{k}$, with non-negative stepsizes $\lambda_{1}^{k}=\left\|z^{k}-x^{k}\right\| \geq 0$ and $\lambda_{2}^{k}=\| v^{k}-$ $w^{k} \| \geq 0$, respectively. We will always assume that, if nonzero, the directions will be normalized to have unitary norm, i.e., $\left\|y^{k}\right\| \in\{0,1\}$ and $\left\|u^{k}\right\| \in\{0,1\}$; indeed, we will only use either $y^{k}=N\left(z^{k}-\bar{x}\right)$ or $y^{k}=0$, and, similarly, either $u^{k}=N\left(v^{k}-\bar{w}\right)$ or $u^{k}=0$. Finally, note that if the direction $y^{k}\left(u^{k}\right)$ is zero, then the corresponding stepsize $\lambda_{1}^{k}\left(\lambda_{2}^{k}\right)$ is irrelevant, thus one can always assume it to be zero.

In the following we will discuss different combinations of conditions $\Upsilon$ over the stepsizes $\lambda_{1}^{k}$ and $\lambda_{2}^{k}$ and conditions $\Psi$ over the directions $y^{k}$ and $u^{k}$, which guarantee the existence of $\left(x^{i}, w^{i}\right)$ satisfying both (3.21) and $\Psi, \Upsilon$ (in step 6 of Sub-procedure 6), as well as the global convergence of Algorithm 7.

\section{The Set of Conditions $R_{1}$}

This section talks about the first choice over combination of $\Psi, \Upsilon$ and its corresponding set of conditions $R_{1}$ :

$$
R_{1} \equiv[(3.20) \wedge \Psi \equiv(3.30) \wedge \Upsilon \equiv(3.31)]
$$

where (3.30) and (3.31) are of the following:

$$
\begin{array}{cl}
y^{k}=N\left(z^{k}-\bar{x}\right), & u^{k}=N\left(v^{k}-\bar{w}\right) \\
\lambda_{1}^{k}=\lambda^{k}\left\|z^{k}-\bar{x}\right\|, & \lambda_{2}^{k}=\lambda^{k}\left\|v^{k}-\bar{w}\right\| .
\end{array}
$$

The rationale of (3.31) is that we want to reduce the relative distance between $z^{k}$ and $v^{k}$ and their respective low points ( $\bar{x}$ and $\left.\bar{w}\right)$ at the same rate; $\lambda^{k} \in[0,1)$ is the fraction of the distance that is travelled, and it must be the same in the $x$-space and in the $w$-space. Furthermore, any two of $x^{k}-\bar{x}, z^{k}-\bar{x}$, and $y^{k}\left(w^{k}-\bar{w}, v^{k}-\bar{w}\right.$, and $\left.u^{k}\right)$ are collinear imply that $x^{k}-\bar{x}, z^{k}-\bar{x}$, and $y^{k}\left(w^{k}-\bar{w}, v^{k}-\bar{w}\right.$, and $\left.u^{k}\right)$ are collinear, i.e. $y^{k}=N\left(x^{k}-\bar{x}\right)=N\left(z^{k}-\bar{x}\right)$ and $u^{k}=N\left(w^{k}-\bar{w}\right)=N\left(v^{k}-\bar{w}\right)$.

The existence of $\left(x^{i}, w^{i}\right)$ satisfying both (3.21) and $\Psi=(3.30), \Upsilon=(3.31)$ (in step 6 of Sub-procedure 6) is obvious: in fact, for $\lambda^{i}=0$ we get $v^{i} z^{i}>1$, 
while for $\lambda^{i}=1$ we get $\bar{w} \bar{x}<1$, and the result follows by continuity (an explicit formula can be easily derived for the correct value of $\lambda^{i}$, but it is of no relevance here).

As previously mentioned in Sub-procedure 6 and remarks of Algorithm 7, the points $\left(z^{k}, v^{k}\right),\left(x^{k}, w^{k}\right)$ satisfy $(3.20), \Psi, \Upsilon$ and therefore $R_{1}$, then $R_{1}$ guarantees the convergence of Algorithm 7 with $\Psi \equiv(3.30)$ and $\Upsilon \equiv(3.31)$ if itself convergent. Recalling that either condition $B_{3}$ or $B_{4}$ allows us to obtain a convergent algorithm; therefore, if both are implied by $R_{1}$, then convergence is surely achieved. A sufficient condition for (2.18) and (2.19) is that movements vanish, i.e.

$$
\lambda_{1}^{k} y^{k} \rightarrow 0, \quad \lambda_{2}^{k} u^{k} \rightarrow 0
$$

Lemma 3.4.4 Under (3.32), both (2.18) and (2.19) hold.

Proof. Due to (2.23), the sequences $\left\{\left(z^{k}, v^{k}\right)\right\}$ and $\left\{\left(x^{k}, w^{k}\right)\right\}$ are bounded; we thus have, using (3.32),

$$
\begin{gathered}
v^{k}\left(z^{k}-x^{k}\right)=v^{k} \lambda_{1}^{k} y^{k} \rightarrow 0, \text { and } \\
v^{k} x^{k}=\left(w^{k}+\lambda_{2}^{k} u^{k}\right) x^{k}=1+\lambda_{2}^{k} u^{k} x^{k} \rightarrow 1,
\end{gathered}
$$

i.e. (2.18). The proof for (2.19) is symmetric.

Of course, if movements vanish so do the difference in objective function value, i.e. (3.32) implies

$$
\left(d z^{k}+e v^{k}\right)-\left(d x^{k}+e w^{k}\right) \rightarrow 0
$$

while the converse need not be true. In fact, (3.33) is easily obtained by

$$
d x^{k}+e w^{k} \leq d z^{k}+e v^{k}(\leq \gamma)
$$

Furthermore, (3.30) and (3.31) ensure that

$$
\left(x^{k}, w^{k}\right)=\lambda^{k}(\bar{x}, \bar{w})+\left(1-\lambda^{k}\right)\left(z^{k}, v^{k}\right)
$$

and this immediately implies (3.34) and then (3.33). Eventually, (3.30) and (3.31) turn out to be sufficient to provide (3.32) as well.

Lemma 3.4.5 If conditions $\Psi \equiv(3.30)$ and $\Upsilon \equiv(3.31)$ hold, then (3.32) holds. 
Proof. Conditions (3.30) and (3.31) imply (3.34), which further gives

$$
d z^{k}+e v^{k} \leq \gamma^{k}=d x^{k-1}+e w^{k-1}
$$

Since $d x^{k}+e w^{k}>\bar{\gamma}$ for all $k$, it follows from (3.36) and (3.34) that $\left\{d x^{k}+e w^{k}\right\}$ is non-increasing and convergent, i.e., $\left(d x^{k-1}+e w^{k-1}\right)-\left(d x^{k}+e w^{k}\right) \rightarrow 0 ;(3.33)$ then follows using (3.36).

From (3.35) we have

$$
d z^{k}-d x^{k}+e v^{k}-e w^{k}=\lambda^{k}\left(d z^{k}-d \bar{x}+e v^{k}-e \bar{w}\right) \geq \lambda^{k}\left(\gamma^{*}-\bar{\gamma}\right)
$$

then together with (3.33) we have $\lambda^{k} \rightarrow 0$. Using the boundedness of $\left\|y^{k}\right\|$ and $\left\|u^{k}\right\|$ this finally gives (3.32).

Therefore, the set of conditions $R_{1}$ implies $B_{3}$ or $B_{4}$, thus it guarantees the convergence for Algorithm 7 with $\Psi \equiv(3.30)$ and $\Upsilon \equiv(3.31)$.

\section{The Set of Conditions $R_{2}$}

This section provides another set of conditions

$$
R_{2} \equiv(3.20) \wedge(3.37)
$$

in which (3.37) is of the following:

$$
\lambda_{1}^{k}>0 \Rightarrow d y^{k} \geq \tau, \quad \lambda_{2}^{k}>0 \Rightarrow e u^{k} \geq \tau
$$

where $\tau$ is a small enough positive value. Let's discuss the convergence property of $R_{2}$.

Lemma 3.4.6 If condition (3.37) holds, then (3.32) holds.

Proof. Condition (3.37) imply that $d z^{k} \geq d x^{k}$ and $e v^{k} \geq e w^{k}$; hence, condition (3.34) holds and from the proof of Lemma 3.4.5 we obtain (3.33). Since both terms are non-negative, this imples $d z^{k}-d x^{k} \rightarrow 0$ and $e v^{k}-e w^{k} \rightarrow 0$.

We want to prove that $\lambda_{1}^{k} \rightarrow 0$ and $\lambda_{2}^{k} \rightarrow 0$, so assume by contradiction that there exists $\theta>0$ such that, by taking subsequences if necessary, $\lambda_{1}^{k} \geq \theta$ for all large enough $k$ (the proof for $\lambda_{2}^{k}$ is analogous); hence, from (3.37) we have $d z^{k}-d x^{k} \geq \lambda_{1}^{k} d y^{k} \geq \tau \theta$ for all (large enough) $k$, a contradiction. 
Lemmas 3.4.4 and 3.4.6 imply that $R_{2} \Rightarrow B_{3} \wedge B_{4}$, hence $R_{2}$ guarantees the global convergence of Algorithm 7. At first glance, it's not obvious how the rather abstract condition (3.37) can be guaranteed; however, when $\Psi \equiv(3.30)$, we can prove that at least a suitable small enough $\tau$ exists.

Lemma 3.4.7 Under $\Psi \equiv(3.30)$, there exists $\tau>0$ such that at each step either $d y^{k} \geq \tau$ or $e u^{k} \geq \tau$.

Proof. Let $M=\sup _{k}\left\{\max \left\{\left\|x^{k}-\bar{x}\right\|,\left\|w^{k}-\bar{w}\right\|\right\}\right\}$; from boundedness of $\left\{\left(x^{k}, w^{k}\right)\right\}, M<+\infty$. Thus, for any $\tau>0$ such that $2 \tau M \leq \gamma^{*}-\bar{\gamma}$ we have

$$
\left(d x^{k}+e w^{k}\right)-(d \bar{x}+e \bar{w}) \geq \gamma^{*}-\bar{\gamma} \geq 2 \tau M \geq \tau\left\|x^{k}-\bar{x}\right\|+\tau\left\|w^{k}-\bar{w}\right\|
$$

which implies that either $d\left(x^{k}-\bar{x}\right) \geq \tau\left\|x^{k}-\bar{x}\right\|$ or $e\left(w^{k}-\bar{w}\right) \geq \tau\left\|w^{k}-\bar{w}\right\|$. The thesis then follows by reminding that, due to (3.30), $y^{k}=N\left(z^{k}-\bar{x}\right)=N\left(x^{k}-\bar{x}\right)$ and $u^{k}=N\left(v^{k}-\bar{w}\right)=N\left(w^{k}-\bar{w}\right)$.

Although a suitable $\tau$ exists, it remains to show how the stepsizes can be chosen to ensure that (3.37); in particular, one has to detect which of the two directions must be given a nonzero stepsize. We propose the following rule:

$$
\left(\lambda_{1}^{i}, \lambda_{2}^{i}\right)= \begin{cases}\left(0, \frac{v^{i} z^{i}-1}{\left(v^{i}-\bar{w}\right) z^{i}}\left\|v^{i}-\bar{w}\right\|\right) & \text { if } d y^{i} \leq e u^{i} \\ \left(\frac{v^{i} z^{i}-1}{v^{i}\left(z^{i}-\bar{x}\right)}\left\|z^{i}-\bar{x}\right\|, 0\right) & \text { otherwise }\end{cases}
$$

It is immediate to verify that the corresponding $\left(x^{i}, w^{i}\right)$ satisfy $w^{i} x^{i}=1$. Relying on $\Psi \equiv(3.30)$ and $\Upsilon \equiv(3.38)$, we can prove that $\left(x^{i}, w^{i}\right)$ and $\left(z^{i}, v^{i}\right)$ exist and satisfy $R_{2}$, then the global convergence of Algorithm 7 is achieved.

Lemma 3.4.8 Under condition (3.26), if $\Psi \equiv(3.30)$ and $\Upsilon \equiv(3.38)$ hold, then (3.21) and (3.37) hold.

Proof. In fact, if $d y^{k} \leq e u^{k}$ then $e v^{k}>e \bar{w}$ (the other case is analogous). The assumption (3.26) allows us to invoke Lemma 3.4.3; by (3.27) we have $\bar{w} z^{k} \leq 1$ whereas $v^{k} z^{k}>1$, and therefore by (3.38) $\lambda_{1}^{k}=0$ and $0<\lambda_{2}^{k} \leq\left\|v^{k}-\bar{w}\right\|$, i.e. (3.21) holds. 
Now, from Lemma 3.4.7 and (3.21) we know that, for a suitably chosen $\tau$,

$$
\text { either } d y^{k} \geq \tau \text { or } e u^{k} \geq \tau \text {. }
$$

Thus, if $d y^{k} \leq e u^{k}$ then surely $e u^{k} \geq \tau$, and from above we know that (3.38) sets $\lambda_{2}^{k}>0$ and $\lambda_{1}^{k}=0$.

\section{The Set of Conditions $R_{3}$}

Another set of conditions is

$$
R_{3} \equiv(3.20) \wedge(3.39)
$$

where (3.39) is of the following:

$$
\begin{aligned}
& \lambda_{1}^{k}=\lambda_{2}^{k}=\lambda^{k} \\
& d y^{k}+e u^{k} \geq \tau
\end{aligned}
$$

for a strictly positive $\tau$.

Lemma 3.4.9 If condition (3.39) holds, then (3.32) holds.

Proof. Condition (3.39) gives, using $\lambda^{k} \geq 0$,

$$
0 \leq \lambda^{k} \tau=\tau\left(\left\|z^{k}-x^{k}\right\|+\left\|v^{k}-w^{k}\right\|\right) / 2 \leq d z^{k}+e v^{k}-d x^{k}-e w^{k}
$$

which implies (3.34). Then, the proof of Lemma 3.4.5 gives

$$
0 \leq \lim _{k \rightarrow \infty} \lambda^{k} \tau \leq \liminf _{k \rightarrow \infty} d z^{k}+e v^{k}-d x^{k}-e w^{k} \leq 0
$$

and therefore $\lambda_{1}^{k}=\lambda_{2}^{k}=\lambda^{k} \rightarrow 0$.

Lemmas 3.4.4 and 3.4.9 guarantee global convergence of $R_{3}$. Again, an implementable version of the abstract condition (3.39) is required; we propose $\Upsilon \equiv(3.39)(a)$ and $\Psi \equiv(3.40)$ where (3.40) is defined as

$$
y^{i}=\left\{\begin{array}{ll}
N\left(z^{i}-\bar{x}\right) & \text { if } d z^{i}>d \bar{x} \\
0 & \text { otherwise }
\end{array} \quad, \quad u^{i}= \begin{cases}N\left(v^{i}-\bar{w}\right) & \text { if } e v^{i}>e \bar{w} \\
0 & \text { otherwise }\end{cases}\right.
$$

which are enough to give the second part of (3.39). 
Lemma 3.4.10 Condition $\Upsilon \equiv(3.39)(a)$ and $\Psi \equiv$ (3.40) imply (3.39)(b) for some $\tau>0$.

Proof. We argue by contradiction. Define $M<+\infty$ as in the proof of Lemma 3.4.7, and let $\tau=\left(\gamma^{*}-\bar{\gamma}\right) / 2 M ;(3.40)$ guarantees $d z^{k} \geq d x^{k}$ and $e v^{k} \geq e w^{k}$, hence it suffices to show $d y^{k} \geq \tau$ or $e u^{k} \geq \tau$.

By definition, $d x^{k}+e w^{k}-\bar{\gamma} \geq 2 \tau M \geq \tau\left(\left\|w^{k}-\bar{w}\right\|+\left\|w^{k}-\bar{w}\right\|\right)$, then either $d x^{k}-d \bar{x} \geq \tau\left\|x^{k}-\bar{x}\right\|$ or $e w^{k}-e \bar{w} \geq \tau\left\|w^{k}-\bar{w}\right\|$. Recalling that $x^{k} \in\left(\bar{x}, z^{k}\right]$ and $w^{k} \in\left(\bar{w}, v^{k}\right]$, the thesis follows.

Therefore, the points generated by Algorithm 7 with $\Upsilon \equiv(3.39)(a)$ and $\Psi \equiv(3.40)$ satisfy $R_{3}$, then the global convergence is achieved. However, we still have to prove the existence of $\left(x^{k}, w^{k}\right)$ satisfying both (3.21) and $\Upsilon \equiv(3.39)(a)$, $\Psi \equiv(3.40)$ (at step 6 of Sub-procedure 6).

Lemma 3.4.11 Under (3.26) and $\Psi \equiv(3.40)$, there exists a $\lambda^{k}$ satisfying $\Upsilon \equiv$ (3.39)(a) and (3.21).

Proof. Consider the quadratic form $\zeta(\lambda)=u^{i} y^{i} \lambda^{2}-\left(v^{i} y^{i}+z^{i} u^{i}\right) \lambda+v^{i} z^{i}-1$, whose roots are the values of $\lambda$ for which $w^{i} x^{i}=1$. For any $i$ passing step 5 , there are two possibilities: 1) $d z^{i} \leq d \bar{x}$ and then $e v^{i}>e \bar{w}$ (the other case is symmetric). (3.40) chooses $y^{i}=0$ and $x^{i}=z^{i}$. Then $v^{i} x^{i}>1$ and $\bar{w} x^{i} \leq 1$ (guaranteed by Lemma 3.4.3) imply that there exists $w^{i} \in\left[\bar{w}, v^{i}\right)$ such that $w^{i} x^{i}=1$, i.e. (3.21). Set $\lambda_{1}^{i}=\lambda_{2}^{i}=\left\|v^{i}-w^{i}\right\|$, (3.39)(a) follows.

2) $d z^{i}>d \bar{x}$ and then $e v^{i}>e \bar{w}$; using (3.26), Lemma 3.4.3 guarantees $\bar{w} z^{i} \leq 1$ and $v^{i} \bar{x} \leq 1$. Choose $\lambda=\min \left\{\left\|z^{i}-\bar{x}\right\|,\left\|v^{i}-\bar{w}\right\|\right\}$, take $\hat{x}=\bar{x}$ and $\hat{w} \in\left[\bar{w}, v^{i}\right)$ when $\left\|z^{i}-\bar{x}\right\|<\left\|v^{i}-\bar{w}\right\|($ the other case is symmetric). From $\bar{w} \bar{x}<1$ and $v^{i} \bar{x} \leq 1$, we get $\hat{w} \hat{x}<1$, that is $\zeta(\lambda)<0$. Therefore, $\zeta(0)>0$ and the continuity of $\zeta$ guarantees the existence of a positive root $\lambda$ satisfying (3.39)(a) and (3.21).

\section{Other Possibilities of $\Psi$ and $\Upsilon$}

Summarizing the results of the present section, we have developed three different implementable versions of Algorithm 7. While all based on Sub-procedure 6, 
these differ for the direction formula $\Psi$ and the stepsize formula $\Upsilon$. All the variants are succinctly described in Table 3.1 .

\begin{tabular}{|l|l|l|l|}
\hline & $\Upsilon$ & $\Psi$ & other \\
\hline$R_{1}$ & $(3.31)$ & $(3.30)$ & \\
\hline$R_{2}$ & $(3.38)$ & $(3.30)$ & $(3.26)$ \\
\hline$R_{3}$ & $(3.39)(\mathrm{a})$ & $(3.40)$ & $(3.26)$ \\
\hline
\end{tabular}

Table 3.1: Summary of conditions and algorithms providing global convergence

The Table clearly shows that the three algorithms are obtained by properly mixing three different stepsize formulae-(3.31), (3.38) and (3.39)(a) - with two different direction formulae-(3.30) and (3.40); obviously, there are three other possible combinations that have not been discussed so far. These are discussed here, starting with the fourth combination $\Upsilon \equiv(3.38), \Psi \equiv(3.40)$.

It is worth remarking to show the difference and relationship between this algorithm and the algorithm for $R_{2}$ : in some sense, these two algorithms are "equivalent" since they always produce the same $\left(z^{k}, v^{k}\right)$ and $\left(x^{k}, w^{k}\right)$ when starting situations are the same. They share the same stepsize and the same direction when its corresponding stepsize is non-zero. On the other hand, these two algorithms are also "different" since they apply different $\Psi$ and then may have different directions when stepsize is zero. Due to the difference on $\left(y^{k}, u^{k}\right)$, the convergence of this algorithm can not be proved by $R_{2}$, that is why have to introduce another set of conditions $R_{4}$.

Remark 3.4.1 The three approaches $R_{1}, R_{2}$ and $R_{3}$ all obtain, in three different ways, (3.32) and therefore both $B_{3}$ and $B_{4}$. However, in order to get a convergent sequence, only one of $B_{3}$ and $B_{4}$ is required; here we introduce conditions that satisfy only one of the two:

$$
R_{4}(a) \equiv(3.20) \wedge(3.36) \wedge(3.41) \quad \text { and } \quad R_{4}(b) \equiv(3.20) \wedge(3.36) \wedge(3.42)
$$

where (3.41) and (3.42) are of the following ( $\tau$ is any small enough positive value):

$$
\begin{aligned}
& d z^{k} \geq d x^{k} \quad \text { for all } k, \quad(a) \\
& e u^{k} \geq \tau, \quad \text { for all } k, \quad(b) \\
& w^{k} z^{k} \leq 1 \quad \text { for all } k \text {. (c) }
\end{aligned}
$$




$$
\begin{aligned}
& e v^{k} \geq e w^{k} \quad \text { for all } k, \quad(a) \\
& d y^{k} \geq \tau, \quad \text { for all } k, \quad(b) \\
& v^{k} x^{k} \leq 1 \quad \text { for all } k . \quad(c)
\end{aligned}
$$

In fact, under condition (3.36), we have $(3.41) \Rightarrow(2.18)$ and $(3.42) \Rightarrow(2.19)$ : Let's show that $(3.36) \wedge(3.42) \Rightarrow B_{4}$ (the other case is analogous). Conditions (3.36), (3.42)(a) and (3.42)(b) imply (3.33) and thus $\lambda_{1}^{k} \rightarrow 0$, therefore $(2.18)(\mathrm{a})$ holds $\left(\left\{v^{k}\right\}\right.$ is bounded); moreover, $(3.42)(\mathrm{c}) \Rightarrow(2.18)(\mathrm{b})$. Therefore, $R_{4}(a) \Rightarrow B_{3}$ and $R_{4}(b) \Rightarrow B_{4}$, then $R_{4}(a)$ and $R_{4}(b)$ both guarantee convergence of Algorithm 7 .

Lemma 3.4.12 Under (3.26), conditions $\Upsilon \equiv(3.38)$ and $\Psi \equiv(3.40)$ imply (3.21) and (3.41)[(3.42)], for some $\tau>0$.

Proof. We only prove the case $d y^{k} \leq e u^{k}$, as the other case is analogous. In this case, we have $e v^{k}>e \bar{w}$ and thus $\bar{w} z^{k} \leq 1$, which together with (3.38) guarantee (3.21). Rule (3.38) chooses $\lambda_{1}^{k}=0 \Rightarrow z^{k}=x^{k} \Rightarrow z^{k} w^{k}=1 \wedge d z^{k} \geq d x^{k}$, i.e. $(3.41)(\mathrm{a})$ and $(3.41)(\mathrm{c})$ hold.

From the proof of Lemma 3.4.10 and (3.21) we know that, whence using $d y^{k} \leq e u^{k}$, one gets

$$
e u^{k} \geq \max \left\{d y^{k}, e u^{k}\right\} \geq \tau>0
$$

i.e. $(3.41)(\mathrm{b})$ holds.

Lemma 3.4.12 guarantees the existence of $\left(x^{k}, w^{k}\right)$ and global convergence of Algorithm 7 with $\Upsilon \equiv(3.38)$ and $\Psi \equiv(3.40)$. However, we still label this algorithm as "the other possibilities" since it is "equivalent" to the algorithm for $R_{2}$. This is also true for the fifth algorithm, where $\Upsilon \equiv(3.31)$ and $\Psi \equiv(3.40)$.

Lemma 3.4.13 Under (3.26), $\Upsilon \equiv(3.31)$ and $\Psi \equiv(3.40)$ imply (3.21), either (3.30), (3.31) or (3.41) [(3.42)].

Proof. If there are infinite number of $k$ such that $d z^{k}>d \bar{x}$ and $e v^{k}>e \bar{w}$, then rule $(3.40)=(3.30)$. As it has been shown in $\S 4.4 .1,(3.21)$, (3.30) and (3.31) always hold, i.e. the set of conditions $R_{1}$.

Otherwise, assume $d z^{k} \leq d \bar{x}$ and then $e v^{k}>e \bar{w}$ (the other case is symmetric). Rule (3.40) chooses $y^{k}=0$ and thus $x^{k}=z^{k}$, which implies (3.41)(a) and 
(3.41)(c). $v^{k} z^{k}>1$ and $\bar{w} z^{k} \leq 1$ (guaranteed by Lemma 3.4.3) imply $w^{k} \in\left[\bar{w}, v^{k}\right]$, (3.21) follows. Then $e u^{k} \geq \tau$, i.e. $(3.41)(\mathrm{b})$.

Lemma 3.4.13 implies that the points $\left(z^{k}, v^{k}\right)$ and $\left(x^{k}, w^{k}\right)$, generated by the fifth algorithm, exist and satisfy either $R_{1}$ or $R_{4}$. Furthermore, it always choose the same stepsize, when direction is not zero, with the algorithm for $R_{1}$ and the fourth algorithm. One may can say that, the fifth algorithm is "equivalent" to the algorithm for $R_{1}$ or the fourth, although which one is not known a priori.

Another combination would be $\Upsilon \equiv(3.39)$ (a) and $\Psi \equiv(3.30)$; however, this may lead to the situation where, in Sub-procedure $6, \gamma^{k}<d x^{k}+e w^{k}$, which results in the Algorithm 7 never convergent, as the following example shows.

Example 3.4.1 We consider the (SRP) instance where $n=1, S=\Omega=$ $[-1 / 2,20], Q=\Gamma=[-1 / 2,1 / 2],(\bar{x}, \bar{w})=(0,0), d=1$ and $e=-2$. The optimal solution is $\left(x^{*}, w^{*}\right)=(2,1 / 2)$ and the optimal value is 1 .

Set $\varepsilon=0.9, \bar{\varepsilon}=1, \gamma^{1}=19$; Sub-procedure 6 chooses $\left(z^{1}, v^{1}\right)=\left(\bar{z}^{1}, \bar{v}^{1}\right)=$ $(20,1 / 2)$. The rules (3.39) and (3.30) choose $y^{1}=u^{1}=1$ and $\lambda_{1}^{1}=\lambda_{2}^{1} \approx 0.44885$. Then $\left(x^{1}, w^{1}\right) \approx(19.55115,0.05115)$ and $d x^{1}+e w^{1} \approx 19.44885$. Then Subprocedure 6 stops and report $\gamma^{2} \approx 19.44885>\gamma^{1}$, and iterate.

Comparison Between $R_{1}, R_{2}, R_{3}$ and $R_{4}$

We now proceed to show that all these conditions are indeed different, i.e., given the same instance and the same starting conditions they have different optimizing sequences.

Example 3.4.2 We consider the (SRP) instance where

$$
\begin{gathered}
\Gamma^{*}=\left\{\left(x_{1}, x_{2}\right) \mid x_{1}^{2}+x_{2}^{2} \leq 4\right\} \\
\Omega=\left\{\left(x_{1}, x_{2}\right) \mid-1 \leq x_{1} \leq 1,-1 \leq x_{2}\right\},
\end{gathered}
$$

$(\bar{x}, \bar{w})=(0,0), d=(0,1)$ and $e=(0,0)$; thus, this actually is a (CDC) instance. The instance is depicted in Figure 3.3. From the Figure, it is clear that the optimal solutions are the intersections between the segments $x_{1}=-1, x_{1}=1$ and the circle, i.e., $x^{*}=(-1, \sqrt{3})$ and $x^{*}=(1, \sqrt{3})$. Therefore, the optimal value is $\gamma^{*}=\sqrt{3} \approx 1.732$. Note that the instance satisfies all standard assumptions: 

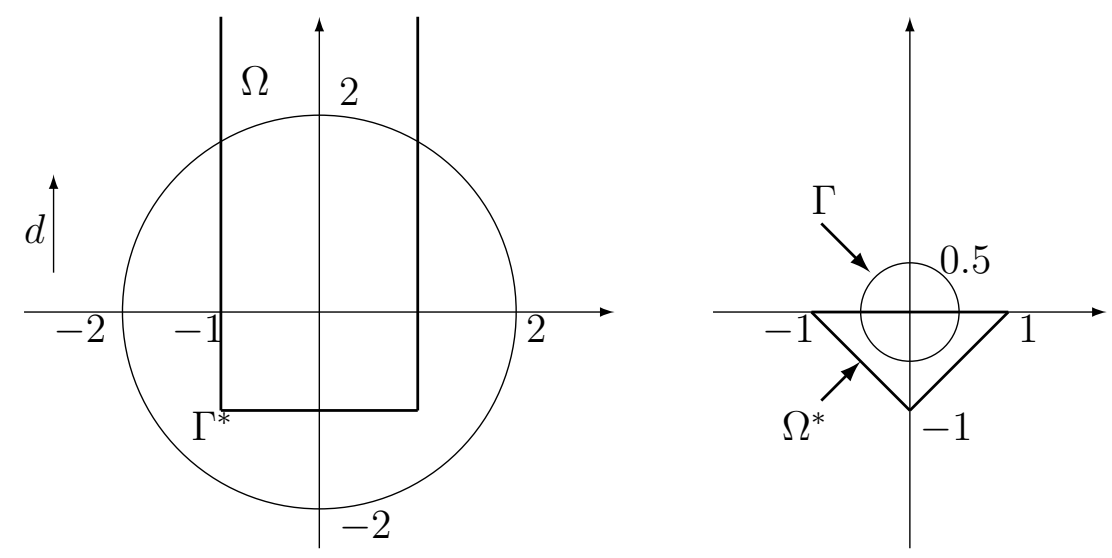

Figure 3.3: Data for Example 3.4.2: $\Omega, \Gamma^{*}, \Omega^{*}$, and $\Gamma$

(3.4), (3.6), (3.8), regularity due to [10, Lemma 2.2] and compactness of $\Gamma^{*}$. Furthermore, the value function of the instance is Lipschitz due to [10, Theorem $3.1]$.

In the polar space, $\Gamma=\left\{\left(w_{1}, w_{2}\right) \mid w_{1}^{2}+w_{2}^{2} \leq 1 / 4\right\}$ and $\Omega^{*}=\left\{\left(w_{1}, w_{2}\right) \mid\right.$ $\left.-w_{1}-w_{2} \leq 1, w_{1}-w_{2} \leq 1, w_{2} \leq 0\right\}$, as depicted in Figure 3.3.

Consider the following convergent sequence:

$$
\gamma^{1}=2, z^{k}=\left(-1, \gamma^{k}\right), x^{k}=\frac{2\left(-1, \gamma^{k}\right)}{\sqrt{1+\left(\gamma^{k}\right)^{2}}}, w^{k}=\frac{\left(-1, \gamma^{k}\right)}{2 \sqrt{1+\left(\gamma^{k}\right)^{2}}} .
$$

Then choose

$$
y^{k}=\frac{z^{k}}{\left\|z^{k}\right\|}=\frac{\left(-1, \gamma^{k}\right)}{\sqrt{1+\left(\gamma^{k}\right)^{2}}}, u^{k}=\frac{w^{k}}{\left\|w^{k}\right\|}=\frac{\left(-1, \gamma^{k}\right)}{\sqrt{1+\left(\gamma^{k}\right)^{2}}} .
$$

Although the choice of $v^{k}$ is not specified, we will always have $d z^{k}+e v^{k}=d z^{k}=$ $\gamma^{k}$, thus condition (3.36) holds.

Case 1: A sequence of points that satisfies $R_{1}$ doesn't satisfy $R_{2}, R_{3}$ and $R_{4}$. Choose $v^{k}=\left(-1, \gamma^{k}\right) / 4$; since

$$
v\left(\overline{R O C}_{\gamma^{k}}\right)=w^{k} z^{k}-1=\sqrt{1+\left(\gamma^{k}\right)^{2}} / 2-1
$$

and $\gamma^{k} \geq \gamma^{*}=\sqrt{3}$ implies

$$
v^{k} z^{k}-1=\left(1+\left(\gamma^{k}\right)^{2}\right) / 4-1 \geq \sqrt{1+\left(\gamma^{k}\right)^{2}} / 2-1
$$


i.e., condition (3.20) holds. We also have $v^{k} /\left\|v^{k}\right\|=w^{k} /\left\|w^{k}\right\|$, hence (3.30) holds. It follows from the definition that

$$
\lambda_{1}^{k}=\sqrt{1+\left(\gamma^{k}\right)^{2}}-2 \quad \text { and } \quad \lambda_{2}^{k}=\left(\sqrt{1+\left(\gamma^{k}\right)^{2}}-2\right) / 4
$$

then

$$
\lambda_{1}^{k} /\left\|z^{k}\right\|=\lambda_{2}^{k} /\left\|v^{k}\right\|=\frac{\sqrt{1+\left(\gamma^{k}\right)^{2}}-2}{\sqrt{1+\left(\gamma^{k}\right)^{2}}},
$$

i.e., condition (3.31) holds. Therefore,

- $R_{1}$ holds: $\left\{\left(z^{k}, v^{k}\right)\right\},\left\{\left(y^{k}, u^{k}\right)\right\}$ and $\left\{\left(x^{k}, w^{k}\right)\right\}$ satisfy conditions (3.20), (3.36), (3.31) and (3.30).

- $R_{2}$ doesn't hold: $\lambda_{2}^{k} \neq 0$ and $e u^{k}=0$.

- $R_{3}$ doesn't hold: $\lambda_{1}^{k} \neq \lambda_{2}^{k}$.

- $R_{4}$ doesn't hold: $v^{k} x^{k}=\sqrt{1+\gamma_{2}^{k}} / 2>1$ for all non-optimal value $\gamma^{k}$ implies that condition (3.42)(c) doesn't hold; $e=0$ implies that condition (3.41)(b) doesn't hold.

Case 2: A sequence of points that satisfies $R_{3}$ doesn't $R_{1}, R_{2}$ and $R_{4}$. Choose $v^{k}=w^{k}+\lambda_{1}^{k} u^{k}$, then we have

$$
v\left(\overline{R O C}_{\gamma^{k}}\right)=\sqrt{1+\left(\gamma^{k}\right)^{2}} / 2-1 \leq v^{k} z^{k}-1=\sqrt{1+\left(\gamma^{k}\right)^{2}}\left(1 / 2+\lambda_{2}^{k}\right)-1
$$

i.e., condition (3.20) holds. Take $\lambda_{1}^{k}=\lambda_{2}^{k}=\sqrt{1+\left(\gamma^{k}\right)^{2}}-2$. Moreover, $d y^{k}+$ $e u^{k}=\gamma^{k} / \sqrt{1+\left(\gamma^{k}\right)^{2}}>1 / 2$. Therefore,

- $R_{3}$ holds: $\lambda_{1}^{k}=\lambda_{2}^{k}$ and $d y^{k}+e u^{k}>1 / 2$ for all $k$, then conditions (3.39) and (3.39b) hold. Furthermore, (3.20) and (3.36) also hold.

- $R_{1}$ doesn't hold: $\lambda_{1}^{k} /\left\|z^{k}\right\| \neq \lambda_{2}^{k} /\left\|v^{k}\right\|$.

- $R_{2}$ doesn't hold: $\lambda_{2}^{k} \neq 0$ and $e u^{k}=0$.

- $R_{4}$ doesn't hold: $v^{k} x^{k}>1$ and $e u^{k}=0$ imply that (3.42)(c)and (3.41)(b) don't hold. 
Case 3: A sequence of points that satisfies $R_{2}$ and $R_{4}$ doesn't satisfy $R_{1}$ and $R_{3}$. The following choices satisfy $R_{2}$ and $R_{4}$ :

$$
v^{k}=w^{k}=\frac{\left(-1, \gamma^{k}\right)}{2 \sqrt{1+\gamma_{2}^{k}}},
$$

We then get

$$
\lambda_{1}^{k}=\sqrt{1+\gamma_{2}^{k}}-2 \text { and } \lambda_{2}^{k}=0, d y^{k}=\gamma^{k} / \sqrt{1+\gamma_{2}^{k}}>1 / 2
$$

Furthermore,

$$
v^{k} z^{k}-1=w^{k} z^{k}-1=v\left(\overline{R O C}_{\gamma^{k}}\right),
$$

i.e., condition (3.20) holds.

- $R_{2}$ holds: (3.20) and (3.36) hold; $\lambda_{2}^{k}=0$ and $d y^{k}>1 / 2$ for all $k$, then condition (3.37) holds.

- $R_{4}$ holds: (3.20) and (3.36) hold; $e u^{k}=0, v^{k} x^{k}=1$ and $d y^{k}>1 / 2$ imply condition (3.42) holds.

- $R_{1}$ and $R_{3}$ don't hold: $\lambda_{1}^{k} \neq 0$ and $\lambda_{2}^{k}=0$.

The residual work is to explore the difference between $R_{2}$ and $R_{4}$.

Example 3.4.3 Let's consider the (SRP) instance where $\Omega=[-1 / 2,20], \Gamma=$ $[-1 / 2,1 / 2], \gamma^{1}=+\infty, d=1$ and $e=-2$. It is easy to check that the optimal solution $\left(x^{*}, w^{*}\right)=(2,1 / 2)$ and the optimal value is $\gamma^{*}=1$.

Case 4: A sequence of points that satisfies $R_{2}$ doesn't satisfy $R_{4}$. Choose $\left(z^{1}, v^{1}\right)=(20,1 / 2),\left(x^{1}, w^{1}\right)=(2,1 / 2),\left(y^{1}, u^{1}\right)=\left(z^{1} /\left\|z^{1}\right\|, v^{1} /\left\|v^{1}\right\|\right)=(1,1)$,

then we get that $\left(\lambda_{1}^{1}, \lambda_{2}^{1}\right)=(18,0)$. This sequence is finite since $\left(x^{1}, w^{1}\right)$ is the optimal point. Since $d y^{1} \geq 1$ and $\lambda_{2}^{1}=0,\left\{\left(z^{1}, v^{1}\right)\right\},\left\{\left(y^{1}, u^{1}\right)\right\}$ and $\left\{\left(x^{1}, w^{1}\right)\right\}$ satisfy $R_{2}$.

- $R_{2}$ holds: $v^{1} z^{1}-1=v\left(\overline{R O C}_{\gamma^{1}}\right)$ and $d z^{1} \leq \gamma^{1}$, i.e., (3.20) and (3.36) hold; $\lambda_{2}^{1}=0$ and $d y^{1}>1 / 2$, then condition (3.37) holds.

- $R_{4}$ doesn't hold: $e u^{1}<0$. 
Case 5: A sequence of points that satisfies $R_{4}$ doesn't satisfy $R_{2}$. Choose

$$
\left(z^{1}, v^{1}\right)=(20,1 / 2),\left(x^{1}, w^{1}\right)=(2,1 / 2),\left(y^{1}, u^{1}\right)=(1,0)
$$

then $\left(\lambda_{1}^{1}, \lambda_{2}^{1}\right)=(18,1)$.

- $R_{4}$ holds: $v^{1} z^{1}-1=v\left(\overline{R O C}_{\gamma^{1}}\right)$ and $d z^{1} \leq \gamma^{1}$, i.e., (3.20) and (3.36) hold; $e u^{1}=0, v^{1} x^{1}=1$ and $e y^{1}=1,\left\{\left(z^{1}, v^{1}\right)\right\},\left\{\left(y^{1}, u^{1}\right)\right\}$ and $\left\{\left(x^{1}, w^{1}\right)\right\}$ satisfy $(3.42)$.

- $R_{2}$ doesn't hold: $\lambda_{2}^{1} \neq 0$ and $e u^{1}=0,\left\{\left(z^{1}, v^{1}\right)\right\},\left\{\left(y^{1}, u^{1}\right)\right\}$ and $\left\{\left(x^{1}, w^{1}\right)\right\}$ don't satisfy (3.37).

These five cases show that $R_{1}, R_{2}, R_{3}$ and $R_{4}$ can not be reduced to each other. Recalling that (CDC) problems are only special cases of the (SRP) problems, then these four ways should provide new ways to solve problem (CDC). However, can these four ways be reduced to those six conditions specialized for problem (CDC)?

Example 3.4.4 Let $\Omega$ and $\Gamma^{*}$ be as defined in (3.44) and (3.43), respectively. Set $d=(0,1), e=(0,0)$.

Choose the sequences of convergent points of Case 1, Case 2 and Case $3,\left\{z^{k}\right\}$ and $\left\{w^{k}\right\}$ are always the same:

$$
w^{k-1} z^{k}=\frac{1+\gamma^{k} \gamma^{k-1}}{2 \sqrt{1+\left(\gamma^{k-1}\right)^{2}}}=\frac{1+\frac{2\left(\gamma^{k-1}\right)^{2}}{\sqrt{1+\left(\gamma^{k-1}\right)^{2}}}}{2 \sqrt{1+\left(\gamma^{k-1}\right)^{2}}}=\frac{\sqrt{1+\left(\gamma^{k-1}\right)^{2}}+2\left(\gamma^{k-1}\right)^{2}}{2+2\left(\gamma^{k-1}\right)^{2}}>1
$$

Therefore, these three sequences of points don't satisfy $C_{3}, C_{4}, D_{1}$ and $D_{2}$. Therefore,

- All these four ways in Problem (SRP) can not be reduced to $C_{3}, C_{4}, D_{1}$ and $D_{2}$.

In the following case, we always choose

$$
\begin{gathered}
z^{k}=\left(-1, \gamma^{k}\right), \delta_{k}=\sqrt{\frac{2}{\sqrt{1+\left(\gamma^{k}\right)^{2}}}}, x^{k}=\delta_{k} z^{k}, w^{k}=\frac{1}{\delta_{k}\left(1+\left(\gamma^{k}\right)^{2}\right)} z^{k}, \\
y^{k}=\frac{z^{k}}{\left\|z^{k}\right\|}=\frac{\left(-1, \gamma^{k}\right)}{\sqrt{1+\left(\gamma^{k}\right)^{2}}}, u^{k}=\frac{w^{k}}{\left\|w^{k}\right\|}=\frac{\left(-1, \gamma^{k}\right)}{\sqrt{1+\left(\gamma^{k}\right)^{2}}}, \varepsilon=\frac{1}{1+1 / \delta_{k}} .
\end{gathered}
$$


- $d z^{k}+e v^{k}=\gamma^{k},(3.36)$ always hold.

- By definition of $v^{k}, u^{k} \equiv v^{k} /\left\|v^{k}\right\|=w^{k} /\left\|w^{k}\right\|$; furthermore, $y^{k}=\frac{z^{k}}{\left\|z^{k}\right\|}$, then conditions (3.30) always hold.

- condition (3.20) holds when $w^{k} \in\left(0, z^{k}\right]$ :

$$
\varepsilon \max \left\{v z-1 \mid(z, v) \in R\left(\gamma^{k}\right)\right\}=\varepsilon\left(1 / \delta_{k}^{2}-1\right)=\frac{1}{\delta_{k}}-1=w^{k} z^{k}-1
$$

Case 6: Choose $v^{k}=w^{k}$, then we have $v^{k} z^{k}-1=\frac{1}{\delta_{k}}-1$. Therefore, Therefore,

- $R_{2}$ holds: (3.36) always hold; $\varepsilon \max \left\{v z-1 \mid(z, v) \in R\left(\gamma^{k}\right)\right\}=w^{k} z^{k}-1 \leq$ $v^{k} z^{k}-1$, (3.20) holds; $d y^{k}>0.5$ and $\lambda_{2}^{k}=0$, (3.37) holds.

- $R_{4}$ holds: (3.20) and (3.36) hold; $e u^{k}=0, v^{k} x^{k}=1$ and $d y^{k}>0.5$ imply that (3.42) holds.

- $C_{1}$ and $C_{2}$ are not satisfied.: $x^{k} \notin \Gamma^{*}$ for all $k$.

Case 7: choose $v^{k}=\frac{\left(-1, \gamma^{k}\right)}{2 \sqrt{1+\left(\gamma^{k}\right)^{2}}}$, then use $\left(x^{k}, w^{k}\right),\left(z^{k}, v^{k}\right)$ and $\left(y^{k}, u^{k}\right)$ we get that

$$
\lambda_{1}^{k}=\left(1-\sqrt{\frac{2}{\sqrt{1+\left(\gamma^{k}\right)^{2}}}}\right)\left\|z^{k}\right\|, \lambda_{2}^{k}=\left(1-\sqrt{\frac{2}{\sqrt{1+\left(\gamma^{k}\right)^{2}}}}\right)\left\|v^{k}\right\| .
$$

Therefore,

- $R_{1}$ holds: (3.36) and (3.30) always hold; $w^{k} \in\left(0, v^{k}\right]$, then (3.20) also holds; $\lambda_{1}^{k} /\left\|z^{k} /\left|=\lambda_{2}^{k} / \| v^{k} /\right|\right.$, then condition (3.31) also holds.

- $C_{1}$ and $C_{2}$ don't hold: $x^{k} \notin \Gamma^{*}$ for all $k$.

Case 8: By choosing $v^{k}=w^{k}+\lambda_{1}^{k} u^{k}$ we will have $\lambda_{1}^{k}=\lambda_{2}^{k}=1-\sqrt{\frac{2}{\sqrt{1+\left(\gamma^{k}\right)^{2}}}}$.

- $R_{3}$ holds: (3.36) always hold; $w^{k} \in\left(0, v^{k}\right]$, then (3.20) also holds; $\lambda_{1}^{k}=\lambda_{2}^{k}$ and $d y^{k} \geq 0.5$ for all $k$, then (3.39) and (3.39b) hold.

- $C_{1}$ and $C_{2}$ don't hold. $x^{k} \notin \Gamma^{*}$ for all $k$. 
Vice versa, the six sets of condition $C_{1}, C_{2}, C_{3}, C_{4}, D_{1}$ and $D_{2}$ can not be reduced to these conditions in Problem (CDC).

Example 3.4.5 Let $\Gamma^{*}$ and $\Omega$ be as defined in (3.43) and (3.44), respectively. Set $(\bar{x}, \bar{w})=0, d=(0,1)$ and $e=(0,0)$. Then

$\Gamma=\left\{\left(w_{1}, w_{2}\right) \mid w_{1}^{2}+w_{2}^{2} \leq \frac{1}{4}\right\}, \Omega^{*}=\left\{\left(w_{1}, w_{2}\right) \mid-w_{1}-w_{2} \leq 1, w_{1}-w_{2} \leq 1, w_{2} \leq 0\right\}$.

The optimal solutions are $(-1, \sqrt{3})$ and $(1, \sqrt{3})$, and the optimal value is $\sqrt{3}$.

Let $S^{1}=Q^{1}=\mathbb{R}^{n}$,

$$
S^{k}=\bigcap_{h<k}\left\{z \mid z w^{h} \leq 1\right\}, Q^{k}=\bigcap_{h<k}\left\{v \mid v x^{h} \leq 1\right\}
$$

$\gamma^{1}=2, z^{k}=\left(-1, \gamma^{k}\right), x^{k}=\frac{2\left(-1, \gamma^{k}\right)}{\sqrt{1+\left(\gamma^{k}\right)^{2}}}, v^{k}=\frac{\left(-1, \gamma^{k}\right)}{4}, w^{k}=\frac{\left(-1, \gamma^{k}\right)}{2 \sqrt{1+\left(\gamma^{k}\right)^{2}}}$.

When $\gamma^{k}$ is not optimal, i.e. $\gamma>\sqrt{3}$, we always have

$$
\zeta\left(w^{k}\right)=\frac{2 \sqrt{1+\gamma_{2}^{k}}-1}{\gamma^{k}}<\gamma^{k}, d x^{k}=\frac{2 \gamma^{k}}{\sqrt{1+\left(\gamma^{k}\right)^{2}}}<\gamma^{k} .
$$

Therefore, nomatter $\gamma^{k+1}=\zeta\left(w^{k}\right)$ or $\gamma^{k+1}=d x^{k}$, we always have

$$
\gamma^{k+1}<d z^{k}=\gamma^{k}
$$

i.e., condition (3.36) holds. Moreover,

$$
v^{k} z^{k}-1=\frac{1+\left(\gamma^{k}\right)^{2}}{4}-1 \geq \frac{\sqrt{1+\left(\gamma^{k}\right)^{2}}}{2}-1=\max \left\{v z-1 \mid(z, v) \in R\left(\gamma^{k}\right)\right\},
$$

that is condition (3.20) holds.

Then we have

$$
w^{i} z^{k}=v^{k} x^{i}=\frac{1+\gamma^{k} \gamma^{i}}{2 \sqrt{1+\gamma_{2}^{i}}}=\frac{\gamma^{k}+\frac{1}{\gamma^{i}}}{\frac{2 \sqrt{1+\gamma_{2}^{i}}-1}{\gamma^{i}}+\frac{1}{\gamma^{i}}}=\frac{\gamma^{k}+\frac{1}{\gamma^{i}}}{\gamma^{i+1}+\frac{1}{\gamma^{i}}} \leq 1
$$

for all $i<k$, that is $z^{k} \in S^{k}$ and $v^{k} \in Q^{k}$. $z^{k}$ and $x^{k}$ are collinear, as well as $v^{k}$ and $w^{k}$. At the end, $x^{k} \in \Omega \cap\left(0, z^{k}\right) \cap \partial \Gamma^{*}$. Therefore, these sequences of points satisfy conditions $C_{1}, C_{2}, C_{3}, C_{4}, D_{1}$ and $D_{2}$. However,

- $R_{2}$ doesn't hold: $e u^{k}=0$ and $\lambda_{2}^{k} \neq 0$.

- $R_{4}$ doesn't hold: $v^{k} x^{k}=w^{k} z^{k}=\frac{\sqrt{1+\gamma_{2}^{k}}}{2}>1$ for all $k$.

- $R_{1}$ and $R_{3}$ don't hold: Choose $\left(y^{k}, u^{k}\right)=\left(z^{k}, 2 v^{k}\right)$, we have $\lambda_{1}^{k}=1-\frac{2}{\left\|z^{k}\right\|}$ and $\lambda_{2}^{k}=0.5-\frac{1}{\left\|z^{k}\right\|}$. 


\subsection{Conclusions}

In this Chapter, we have shown how to extend the oracle-based outer approximation solution methods, developed for (CDC), to the larger class of (SRP) problems. In particular, the main contributions of this Chapter are the following:

- The introduction and investigation of (SRP) problems and their relationships with (CDC) problems; in particular, a through analysis of optimality condition, approximate optimality condition and properties of optimal solutions in (SRP) is performed, as well as the comparison with the corresponding features of (CDC).

- The extension of the concept of approximated oracle to the (SRP) case, which, in contrast to the vertex enumeration techniques usually touted for the $(\mathrm{CDC})$ case, has the potential to make oracle-based algorithms practical even for large-scale instances.

- The development of general hierarchy of conditions ensuring convergence of oracle-based outer approximation algorithms for (SRP).

- A general algorithmic scheme based on the developed hierarchy which leads to three different implementable algorithms; each of them can generate an approximate optimal value in a finite number of steps, where the error can be managed and controlled.

To the best of our knowledge, there are no existing algorithms devoted for (SRP) problems. While (CDC) is just a special case of (SRP) with $e=0$ and $\Gamma=C^{*}$, this apparently minor difference makes for a relevant difference both for the theory and for the algorithms; some crucial properties of (CDC) problems are lost in (SRP) problems, for instance, $x^{*}$ may not in boundary of $\Gamma^{*}$ for any optimal solution $\left(x^{*}, w^{*}\right)$ of problem (SRP). Thus, oracle-based outer approximation algorithms for (CDC) can not be applied to (SRP) directly.

We believe that (SRP), or further appropriate modifications, will be found to contain relevant applications. In particular, the relationship of (SRP) with complementarity problems is currently under study. Thus, we believe that the results in this Chapter constitute a first step towards extending oracle-based outer 
approximation algorithms, originally conceived for DC problems, to a larger class of nonconvex optimization problems. 



\section{Chapter 4}

\section{Conclusions and directions for future work}

Solving nonconvex optimization problems is an extremely challenging task, and exploiting the available structure of the problems is crucial in order to devise efficient solution algorithms. It is well-recognized that formulating the problem at hand in a way that exposes the "right" form of structure is a crucial step towards being able to actually solve it.

DC problems, in particular in their (CDC) form, constitute a very general and expressive class of nonconvex programs where the "source of nonconvexity" is very well identified; the difference of two convex sets, or, alternatively, a single reverse polar constraint. While formulating a nonconvex program as a DC one allows to apply the powerful techniques of convex analysis to the "convex components" of the problem in order to devise solution algorithms, an issue of this choice is that the nonconvexity in these problems lies in the feasible set.

It is widely accepted that a non-convex objective function is somewhat "less difficult" than a non-convex constraint; while one is confronted with the problem of local minima, many heuristic techniques can be applied to find "good" solutions in practice, and powerful relaxation techniques can be used to bound the gap between the obtained solution and the true optimum. By contrast, obtaining even a feasible solution to a problem with a nonconvex feasible region can be an extremely challenging task.

Therefore, we believe that oracle-based algorithms like those studied in this Thesis make sense, as they move the non-convexity from the constraints to the 
objective function. It is true that the "main source of difficulty" of these algorithms lies in the oracle; however, even using an appropriate oracle to find an optimal solution to the original problem is not straightforward. Even more so when approximations have to be taken into account, and this is necessary if any hope that these approaches can be computationally viable in practice is to be retained; while solving the oracle exactly (several times) is clearly hopeless in practice for large-scale problems, approximated oracles like those proposed in this Thesis have the potential to make these approaches practical.

The main contributions of the present Thesis are the following:

- the introduction of the concept of "approximate oracle" for the optimality conditions of (CDC) and (SRP) rewritten in optimization form;

- a thorough study of the impact of approximations in the optimality conditions onto the quality of the approximate optimal solutions satisfying them;

- the development of very general hierarchies of convergence conditions, similar but not identical for (CDC) and (SRP), starting from very abstract ones (fully exploitating the "primal-polar" in the (CDC) case) and moving towards more readily implementable ones;

- the proposal of general algorithmic schemes, based on the developed hierarchies, giving rise to many different implementable algorithms, which can be proven to generate an approximate optimal value in a finite number of steps, where the error can be managed and controlled;

- the definition and study of the theoretical properties of the new class of (SRP) problems, properly generalizing (CDC).

While this Thesis seem to offer a quite comprehensive convergence theory for oracle-based outer approximation algorithms for (CDC) and (SRP) problems, much still needs to be done before these algorithms become widely used and accepted as those based on the branch and bound paradigm. In particular, we identify the following set of promising research lines: 
- Identify practically efficient ways for implementing the oracle, which is the computational bottleneck of our algorithms, for cases where $\Omega$ and $C$ $(\Gamma)$ have further exploitable forms of structure. One prominent example could be combinatorial optimization problems, which are usually modeled by means of convex problems with integrality constraints like $y \in\{0,1\}$. It is well-known that these problems can be reformulated as DC problems (in the specific case by means of the DC constraints $y-y^{2} \leq 0, y \in$ $[0,1])$. As there many existing algorithms devoted to global maximization problems with a non-concave objective function and convex constraints, we believe it possible to find appropriate algorithms that approximately solve the "oracle" in an efficient way for (CDC) or (SRP) problems with specific structures.

- While polyhedra, based on linear cuts, are the simplest and by far most employed form of approximation of generic convex sets, nonlinear approximations are gaining traction in the recent years, with a huge development e.g. in SemiDefinite programs. We believe that nonlinear approximations may turn out to be beneficial, especially for problems with specific structure, but the convergence theory of the algorithms will need to be substantially revised to accommodate them.

- All the algorithms in this Thesis are restricted to problems with bounded feasible sets, as this gives properties crucial for the convergence analysis of the algorithms. However, (CDC) and (SRP) problems with unbounded feasible sets exist and may have practical interest, so the extension of the present theory to cover the unbounded case may be of some interest.

- Last but not least, further studies on (SRP) are needed; in particular, the relationship between the latter class and complementarity problems need be analyzed. It is possible that this study will allow us to extend the oracle-based outer approximation algorithms to other relevant classes of optimization problems, thus giving the results of this Thesis a broader sense and impact. 



\section{Bibliography}

[1] A.D. Alexandrov, "On surfaces which may be represented by a difference of convex functions", Izvestiya Akademii Nauk Kazakhskoj SSR, Seria Fiziko Matematicheskikh, 3 (1949), 3-20.

[2] L. Altangerel, C.Altannar, R. Enkhbat, "A note on the connection of variational ineualities with convex and concave programming", Mong. Math. J. 9 (2005), 2-8.

[3] M. Andramonov, A. Rubinov, B. Glover, "Cutting angle methods in global optimization", Appl. Math. Lett. 12 (1999), 95-100.

[4] S. Bali, "Minimization of a concave function on a bounded convex polyhedron", Ph.D. Dissertation, University of California at Los Angeles, Los Angeles, (1973).

[5] V.T. Ban, "A finite algorithm for minimizing a concave function under linear constraints and its applications", IFTP Working Conference on Recent Advances on System Modelling and Optimization. Hanoi, (1983).

[6] S. Ben Saad, S.E. Jacobsen, "A level set algorithm for a class of reverse convex programs", Ann. Oper. Res. 25 (1990), 19-42.

[7] S. Ben Saad, S.E. Jacobsen, "A new cutting plane algorithm for a class of reverse convex 0-1 integer programs", Recent advances in global optimization, Princeton University Press, Princeton, NJ. (1992), 152-164.

[8] S. Ben Saad, S.E. Jacobsen, "Comments on a reverse convex programming algorithm", J. Global Optim. 5 (1994), 95-96. 
[9] H.P. Benson, "Concave minimization: theory, applications and algorithms", Handbook of global optimization, 43-148, Nonconvex Optim. Appl., 2, Kluwer Acad. Publ., Dordrecht, 1995.

[10] G. Bigi, A. Frangioni, Q.H. Zhang, "Outer approximation algorithms algorithms for canonical DC problems", TR-08-04, Dipartimento di Informatica, Università di Pisa (2008).

[11] G. Bigi, A. Frangioni, Q.H. Zhang, "Outer approximation algorithms for Canonical Reverse Polar problems", TR-0\%-0\%, Dipartimento di Informatica, Università di Pisa (2007).

[12] R. Blanquero, E. Carrizosa, "On covering method for D.C. optimization", J. Global Optim. 18 (2000), 265-274.

[13] R. Blanquero, E. Carrizosa, "A D.C. biobjective local model", J. Global Optim. 23 (2002), 139-154.

[14] M. Borchardt, O. Engel, "A counterexample to a global optimization algorithm", J. Global Optim. 5 (1994), 371-372.

[15] M.C. Bohringer, S.E. Jacobsen, "Converent cutting planes for linear programs with additional reverse convex constraints", Lecture Notes in Control and Inform. Sci. 59 Springer, Berlin, (1984), 263-272.

[16] K. Breiman, A. Cutler, "A deterministic algorithm for global optimization", Math. Program. 58 (1993), 179-199.

[17] A.V. Cabot, "Variations on a cutting plane method for solving concave minimization problems with linear constraints", Naval Res. Logist. Quart. 21 (1974), 265-274.

[18] E.W. Cheney, A.A. Goldstein, "Newton's method for convex programming and Tchebyshell approximation", Numer. Math. 1 (1959), 253-268.

[19] A. Chinchuluun, E. Rentsen, P.M. Pardalos, "A numerical method for concave programming problems", Appl. Optim. 99 (2005), 251-273.

[20] D.Z. Du, P.M. Pardalos, "Minimax and Applications (Nonconvex Optimization and Its Applications)", Springer 1 edition (1995). 
[21] J. Duba, L. Vesely, L. Zajicek, "On D.C. Functions And Mappings", Atti Sem. Mat. Fis. Univ. Modena 51 (2003), 111-138.

[22] R. Enkhbat, "On some theory, methods and algorithms for concave programming", Optimization and optimal control, Ser. Comput. Oper. Res. World Sci. Publ., River Edge, NJ, (2003), 79-102.

[23] J.E. Falk, K.R. Hoffman, "A successive underestimation method for concave minimization problems", Math. Oper. Res. 1 (1976), 251-259.

[24] J.E. Falk, K.R. Hoffman, "Concave Minimization via collapsing polytopes", Oper. Res. 34 (1986), 919-929.

[25] J.E. Falk, R.M. Soland, "An algorithm for separable nonconvex programming problems", Manag. Sci. 15 (1969), 550-569.

[26] A. Ferrer, "Representation if a polynomial function as a difference of convex polynomials, with an application", Lecture Notes in Economics and Mathematical Systems, (2001), 189-207.

[27] J. Fulop, "A finite procedure to generate feasible points for the extreme point mathematical programming problem", European J. Oper. Res. 35 (1988), $228-241$.

[28] J. Fulop, "A finite cutting plane method for solving linear programs with an additional reverse constraint", European J. Oper. Res. 44 (1990), 395-409.

[29] J. Fulop, "Lagrangian duality of concave minimization subject to linear constraints and an additional facial reverse convex constraint", J. Optim. Theory Appl. 91 (1996), 617-644.

[30] R.E. Gomory, "Outline of an algorithm for integer solutions to linear programs", Bull. Amer. Math. Soc. 64 (1958), 275-278.

[31] R.E. Gomory, "Solving linear programming problems in integers", in R. Bellman, M. Hall (eds), Combinatorial Analysis, American Mathematical Society, Rhode Island (1960), 211-215.

[32] P. Hartman, "On functions representable as a difference of convex functions", Pacific J. Math. 9 (1959), 707-713. 
[33] R.J. Hillestad, S.E. Jacobsen, "Linear programs with an additional reverse convex constraint" Appl. Math. Optim. 6 (1980) 257-269.

[34] R.J. Hillestad, S.E. Jacobsen, "Reverse convex programming", Appl. Math. Optim. 6 (1980), 63-78.

[35] J.B. Hiriart-Urruty, "Generalized differentiability, duality and optimization for problems dealing with difference of convex functions", in M. Beckmann, W. Krelle (eds.), Convexity and Duality in Optimization, Lecture notes in Economics and Mathematical Systems, 256, Springer, Berlin (1985), 37-70.

[36] J.B. Hiriart-Urruty, "From convex optimization to nonconvex optimization. Necessary and sufficient conditions for global optimality", in F. H. Clarke, V. F. Demcprime yanov and F. Giannessi (eds), Ettore Majorana International Science Series: Physical Sciences, 43 , Plenum Press, New York, 1989.

[37] J.B. Hiriart-Urruty, C. Lemaréchal, Convex Analysis and Minimization Algorithms, Springer-Verlag, 1993.

[38] L.T. Hoai An, P.D. Tao, "A continuous approach for globally solving linearly constrained quadratic zero-one programming problems", Optimization 50 (2001), 93-120.

[39] L.T. Hoai An, P.D. Tao, "Solving a class of linearly constrained indefinie quadratic problems by D.C. algorithms", J. Global Optim. 11 (1997), 253285.

[40] L.T. Hoai An, P.D. Tao, "The DC(difference of convex functions) programming andDCArevisited withDCmodels of realworld nonconvex optimization problems", Ann. Oper. Res. 133 (2005), 23-46.

[41] R. Horst, "An algorithm for nonconvex programming problems", Math. Program. 10 (1976), 312-321.

[42] R. Horst, P.M. Pardalos, Handbook of global optimization, Kluwer Academic Publishers, Dordrecht (1995). 
[43] R. Horst, N.V. Thoai, "Modification, implementation and comparison of three algorithms for globally solving linearly constrained concave minimization problems", Computing, 42 (1989), 271-289.

[44] R. Horst, N.V. Thoai, "Erratum:"Modification, implementation and comparison of three algorithms for globally solving linearly constrained concave minimization problems", Computing 59 (1997), 271-289.

[45] R. Horst, H. Tuy, Global optimization, Springer, Berlin, (1990).

[46] R. Horst, H. Tuy, "Global optimization: deterministic approaches", Springer-Verlag, Berlin, (1993).

[47] R. Horst, H. Tuy, "Global optimization: deterministic approaches", Springer, 3rd edition, (1996).

[48] R. Horst, T.Q. Phong, N.V. Thoai, "On solving general reverse programming problems by a sequence of linear programs and line searches", Ann. Oper. Res. 25 (1990), 1-18.

[49] R. Horst, N.V. Thoai, H.P. Benson, "Concave minimization via conical pratitions and polyhedral outer approximation", Math. Programming 50 (1991), 259-274.

[50] R. Horst, N.V. Thoai, H. Tuy, "Outer approximation by polyhedral convex set", Oper. Res. Spektrum. 9 (1987), 153-159.

[51] R. Horst, N.V. Thoai, H. Tuy, "On an outer approximation concept in global optimization", Optim. 20 (1989), 255-264.

[52] R. Horst, T.Q. Phong, N.V. Thoai, J.D. Vries, "On solving a D.C. programming problem by a sequence of linear programs", J. Global Optim. 2 (1991), $183-203$.

[53] K.L. Hoffman, "A method for globally minimizing concave functions over convex sets", Math. Program. 20 (1981), 22-32.

[54] S.E. Jacobsen, "Convergence of a Tuy-type algorithm for concave minimization subject to linear inequality constraints", Appl. Math. Optim. 7 (1981) $1-9$. 
[55] S.E. Jacobsen, K. Moshirvaziri, "Computational experience using an edge search algorithm for linear reverse convex programs", J. Global Optim. 9 (1996), 153-167.

[56] V. Jeyakumar, B.M. Glover, "Characterizing global optimality for DC DC optimization problems under convex inequality constraints", J. Global Optim. 8 (1996), 171-187.

[57] A. H. G. Rinnooy Kan, G. T. Timmer, "Stochastic global optimization methods part I: Clustering methods", Math. Program. 39 (1987), 27-56.

[58] A. H. G. Rinnooy Kan, G. T. Timmer, "Stochastic global optimization methods part II: Multi level methods", Math. Program. 39 (1987), 57-78.

[59] J.E.Jr. Kelley, "The cutting plane method for solving convex programs", J. Soc. Indust. Appl. Math. 8 (1960), 703-712.

[60] H. Konno, A. Wijayanayake, "Porfolio optimization under D.C. transaction costs and minimal transaction unit constraints", J. Global Optim. 22 (2002), 137-154.

[61] T. Kuno, H. Nagai, "A simplicial algorithm with two-phase bounding operation for a class of concave minimization problems", Pac. J. Optim. 1 (2005), 297-313.

[62] S.M. Liu, G.P. Papavassilopoulos, "Algorithms for globally solving D.C. minimization problems via concave programming", Proceedings of the American Control Conferences, Seattle, Washington, (1995).

[63] P. Marcotte, S.Q. Wu, "Finding the strictly local and $\varepsilon$-global minimizers of concave minimization with linear constraints", J. Comput. Math. 15 (1997), 327-334.

[64] P. McKeown, "A vertex ranking procedure for solving the linear fixed charge problem", Oper. Res. 23 (1975), 1182-1191.

[65] C. Meyer, "A simple finite cone covering algorithm for concave minimization", J. Global Optim. 18 (2000), 357-365. 
[66] C.Meyer, "On Tuy's 1964 cone splitting algorithm for concave minimization", Nonconvex Optim. Appl. 53 Kluwer Acad. Publ., Dordrecht, (2001).

[67] K. Moshirvaziri, M.A. Amouzegar, "A subdivisional scheme for linear programs with an additional reverse convex constraint", Asia-Pacific J. Oper. Res. 15 (1998), 179C192.

[68] K. Moshirvaziri, M.A. Amouzegar, "A cutting plane algorithm for linear reverse convex programs" Ann. Oper. Res. 105 (2001), 201-212.

[69] K.G. Murty, "Solving the fixed charge problem by ranking the extreme points", Oper. Res. 16 (1968), 268-279.

[70] L.D. Muu, "A convergent algorithm for solving linear programs with an additional reverse convex constraint", Kybernetika 21 (1985), 428-435.

[71] M. Nast, "Subdivision of simplices relative to a cutting plane and finite concave minimization", J. Global Optim. 9 (1996), 65-93.

[72] M.D. Nghia, N.D. Hieu, "A method for solving reverse convex programming problems", Acta Math. Vietnam. 11 (1986), 241-252.

[73] P.M. Pardalos, H.E. Romeijn, H.Tuy, "Recent Developments and Trends in Global Optimization", J. Comp. App. Math, 124 (2000), 209-228.

[74] J.P. Penot, "What is quasiconvex analysis?", Optimization 47 (2000), 35110.

[75] J.P. Penot, M.L. Bougeard, "Approximation and decomposition properties of some classes of locally d.c. functions", Math. Program. 41 (1988), 195-227.

[76] T.Q. Phong, P.D. Tao, L.T. Hoai An, "A method for solving D.C. programming problems; application to fuel mixture nonconvex optimization problem", J. Global Optim. 6 (1995), 87-105.

[77] M. Porembski, "How to extend the concept of convexity cuts to derive deeper cutting planes", J. Global Optim. 15 (1999), 371-404. 
[78] M. Porembski, "Cone adaptation strategies for a finite and exact cutting plane algorithm for concave minimization", J. Global Optim. 24 (2002), 89107.

[79] M. Porembski, "A new successive partition algorithm for concave minimization based on cone decomposition and decomposition cuts", J. Global Optim. 29 (2004), 191-224.

[80] R.T. Rockafellar, Convex Analysis, Princeton University Press, 1970.

[81] J.B. Rosen, "Global minimization of a linearly constrained concave function by partition of feasible domain", Math. Oper. Res. 8 (1983), 215-230.

[82] D.S. Rubin, "Vertex ranking and cardinality constrained linear programs", Oper. Res. 23 (1975), 555-565.

[83] A.I. Rusakov, "Concave programming under the simplest linear restrictions", Zh. Vychisl. Mat. Mat. Fiz. 43 (2003), 951-960.

[84] S. Sen, H.D. Sherali, "Nondifferentiable reverse convex programs and facetial convexity cuts via a disjunctive characterization", Math. Programming. 37 (1987) 169-183.

[85] A.S. Strekalovsky, "On convergence of a global search strategy for reverse convex problems", J. Appl. Math. Decis. Sci. 3 (2005), 149-164.

[86] A.S. Strekalovsky, "On a local search for reverse convex problems", Global Optimization, 33-43, Nonconvex Optim. Appl. 84 Springer, New York, (2006).

[87] A.S. Strekalovsky, I. Tsevendorj, "Testing the $\mathbb{R}$-strategy for a reverse convex problem", J. Global Optim. 13 (1998), 61-74.

[88] H.A. Taha, "Concave minimization over a convex polyhedron", Naval Res. Logist. Quart. 20 (1973), 533-548.

[89] P.D. Tao, "Algorithms for Solving a Class of Non Convex Optimization Problems. Methods of Subgradients", Mathematics for Optimization, Fermat Days 85. Elsevier Science Publishers, B.V. North- Holland, (1986). 
[90] P.D. Tao, S. El Bernoussi, "Numerical methods for solving a class of global nonconvex optimization problems", International Series of Numerical Mathematics 87 (1989), 97-132.

[91] P.T. Thach, "Convex programs with several additional reverse convex constraints", Acta Math. Vietnam. 10 (1985), 35-57.

[92] P.T. Thach, "D.c sets, d.c. functions and nonlinear equations", Math. Program. 58 (1993), 415-428.

[93] P.T. Thach, "Non-discriminating prices in linear programs with a reverse convex constraint", Pac. J. Optim. 2 (2005), 347-355.

[94] T.V. Thieu, "A finite method for globally minimizing a concave function over an unbounded polyhedral convex sets and its applications", Acta Math. Hungar. 52 (1988), 21-36.

[95] N.V. Thoai, "A modified version of Tuy's method for solving d.c. programming problems", Optimization 19 (1988), 665-674.

[96] N.V. Thuong, H. Tuy, "A finite algorithm for solving linear programs with an additional reverse convex constraint", Lecture Notes in Econom. and Math. Systems. 255 Springer, Berlin, (1985), 291-302.

[97] I. Tseveendorj, "Reverse convex problems: an approach based on optimality conditions", J. Appl. Math. Decis. Sci. (2006), 1-16.

[98] H.D. Tuan, "Remarks on an algorithm for reverse convex programs", $J$. Global Optim. 16 (2000), 295-297.

[99] H. Tuy, "Concave programming under linear constrains", Soviet. Math. 5 (1964), 1437-1440.

[100] H. Tuy, "On outer approximation methods for solving concave minimization problems", Acta Math. Vietnam. 8 (1983), 3-34.

[101] H. Tuy, "A general deterministic approach to global optimization via d.c. programming", in J.B. Hiriart-Urruty (ed.) FERMAT Days 85: Mathematics for Optimization, North-Holland, Amsterdam (1986), 273-303. 
[102] H. Tuy, "Global minimization of a difference of two convex functions", Math. Programming Studies 30 (1987), 150-182.

[103] H. Tuy, "An implicit space covering method with applications to fixed point and global optimization problems", Acta Math. Vietnam. 12 (1987), 93-102.

[104] H. Tuy, "Convex programs with an additional reverse convex constraint", J. Optim. Theory Appl 52 (1987), 463-486.

[105] H. Tuy, "Global minimization of a concave function subject to mixed linear and reverse convex constraints", Acta Math. Vietnam. 13 (1988) 3-19.

[106] H. Tuy, "On polyhedral annexation method for concave minimization", in L.J. Leifman (eds.) Functional analysis, optimization and mathematical economics, Oxford University Press, New York, (1990), 248-260.

[107] H. Tuy, "Normal conical algorithm for concave minimization over polytopes", Math. Programming 51 (1991), 229-245.

[108] H. Tuy, "Effect of the subdivision strategy on convergence and efficiency of some global optimization algorithms", J. Global Optim. 1 (1991), 23-36.

[109] H. Tuy, "Polyhedral annexation, dualization and dimension heduction technique in global optimization", J. Global Optim. 1 (1991), 229-244.

[110] H. Tuy, "On nonconvex optimization problems with separated nonconvex variables", J. Global Optim. 2 (1992), 133-144.

[111] H. Tuy, "The complementary convex structure in global optimization", $J$. Global Optim. 2 (1992), 21-40.

[112] H. Tuy, "Canonical DC programming problem: outer approximation methods revisited", Oper. Res. Lett. 18 (1995), 99-106.

[113] H. Tuy, "D.C. optimization: theory, methods and algorithms", in R. Horst, P.M. Pardalos (eds.), Handbook of global optimization, Kluwer Academic Publishers, Dordrecht (1995), 149-216.

[114] H. Tuy, "Convex programs with an additional reverse convex constraint", J. Optim. Theory Appl. 52 (1997), 463-486. 
[115] H. Tuy, Convex Analysis and Global Optimization, Kluwer Academic Publishers, 1998.

[116] H. Tuy, "On some recent advances and applications of D.C. optimization", in Optimization V.H. Nguyen, J.J. Strodiot, P. Tossings, (eds.), Lecture Notes in Economics and Mathematical Systems Springer, 481 (2000), 473497.

[117] H. Tuy, "On global optimality conditions and cutting plane algorithms", J. Optim. Theory Appl. 118 (2003), 201-216.

[118] H. Tuy, "Counter-examples to some results on D.C. optimization", www.math.ac.vn/library/download/e-print/03/ps/htuy18.ps.

[119] H. Tuy, F.A. Al-Khayyal, "Global optimization of a nonconvex single facility location problem by sequential unconstrained convex minimization", J. Global Optim. 2 (1992), 61-71.

[120] H. Tuy, N.D. Nghia, "Decomposition algorithm for reverse convex programs", Vietnam J. Math. 28 (2000), 43-55.

[121] H. Tuy, B.T. Tam, "Polyhedral annexation vs outer approximation for the decomposition of monotonic quasiconcave minimization problems", Acta Math. Vietnam. 20 (1995), 99-114.

[122] H. Tuy, F. Al-Khayyal, F.J. Zhou, "A D.C. optimization method for single facility location problems", J. Global Optim. 7 (1995), 209-227.

[123] H. Tuy, A. Migdalas, P. Varbrand, "A quasiconcave minimization method for solving linear two-level programs", J. Global Optim. 4 (1994), 243C263.

[124] A.F. Veinott, "The supporting hyperplane method for unimodal programming", Oper. Res. 15 (1967), 147-152.

[125] L. Vesley, L. Zajicek, "Delta-convex mappings between Banach space and applications", Dissertations Math. Rozprawy Mat. 289 (1989).

[126] Q. Wei, H. Yan, "An algebra based approach for linearly constrained concave minimization", Comput. Math. Appl. 43 (2002), 965-974. 
[127] S.Yamada, T.Tanino, M. Inuiguchi, "An inner approximation method incorporating with a penalty function method for a reverse convex programming problem". J. Comput. Appl. Math. 146 (2002), 57C75.

[128] S.Yamada, T.Tanino, M. Inuiguchi, "Inner approximation method for a reverse convex programming problem". J. Optim. Theory Appl. 107 (2000), $355 \mathrm{C} 389$.

[129] P.B. Zwart, "Nonlinear programming: counterexamples to two global optimization algorithms", Oper. Res. 21 (1973), 1260-1266.

[130] P.B. Zwart, "Global Maximization of a convex function with linear inequality constraints", Oper. Res. 22 (1974), 602-609. 